ESAIM: PROCEEDINGS, April 2007, Vol.17, 96-142

Alain Piétrus \& Michel H. Geoffroy, Editors

\title{
DUALITY METHODS FOR THE STUDY OF HAMILTON-JACOBI EQUATIONS
}

\author{
Jean-Paul PENOT ${ }^{1}$ And Michel VOLLE ${ }^{2}$
}

\begin{abstract}
We present a survey of recent results about explicit solutions of the first-order HamiltonJacobi equation. We take advantage of the methods of asymptotic analysis, convex analysis and of nonsmooth analysis to shed a new light on classical results. We use formulas of the Hopf and Lax-Oleinik types. In the quasiconvex case the usual Fenchel conjugacy is replaced by quasiconvex conjugacies known for some years and the usual inf-convolution is replaced by a sublevel convolution. Inasmuch we use weak generalized convexity and continuity assumptions, some of our results are new; in particular, we do not assume that the data are finite-valued, so that equations derived from attainability or obstacle problems could be considered.
\end{abstract}

Résumé. Nous présentons un survol de quelques résultats récents relatifs aux solutions explicites des équations d'Hamilton-Jacobi du premier ordre. Nous nous servons des méthodes de l'analyse asymptotique, de l'analyse convexe et de l'analyse non lisse pour apporter une lumière nouvelle sur des résultats classiques. Nous utilisons des formules de type Hopf et Lax-Oleinik. Dans le cas quasiconvexe, la conjugaison classique de Fenchel est remplacée par des conjugaisons connues depuis quelques années et l'inf-convolution est remplacée par la convolution en sous-niveaux. Comme nous faisons des hypothèses de convexité généralisée et de continuité assez générales, certains des résultats présentés sont nouveaux; en particulier, nous ne supposons pas que les données ne prennent que des valeurs finies, de sorte que des équations provenant de problèmes d'atteignabilité ou de problèmes d'obstacles pourraient être considérés.

\section{INTRODUCTION}

The Hamilton-Jacobi equation has been extensively studied during the last decades (see [16]- [51], [55][58], [60]- [63], [70]- [76], [112], [122], [125]- [128]...) and several monographs ( [13], [16], [34], [51], [75], [122]) are devoted to it, entirely or partially. In particular, the key notion of viscosity solution has given a new start to existence and uniqueness questions ( [40], [39], [37], [16], [13], [51]...).

Given a normed vector space (n.v.s.) $X$, with dual $X^{*}$ and functions $g: X \rightarrow \overline{\mathbb{R}}:=\mathbb{R} \cup\{-\infty,+\infty\}$, $H: X^{*} \times \mathbb{R} \rightarrow \overline{\mathbb{R}}$, the evolution Hamilton-Jacobi equation consists in finding solutions to the system

$$
\begin{array}{rlrl}
\forall(x, t) & \in X \times \mathbb{P} & \frac{\partial u}{\partial t}(x, t)+H(D u(x, t), u(x, t))=0 \\
\forall x & \in X & u(x, 0)=g(x),
\end{array}
$$

\footnotetext{
1 Université de Pau, Faculté des Sciences, Laboratoire de Mathématiques Appliquées, CNRS UMR 5142 Av. de l'Université 64000 PAU, France.

2 Université d'Avignon, Faculté des Sciences, Département de Mathématiques, 33 rue Louis Pasteur 84000 AVIGNON, France.
}

(C) EDP Sciences, SMAI 2007 
where $u: X \times \mathbb{R}_{+} \rightarrow \overline{\mathbb{R}}$ is the unknown function, $D u$ (resp. $\frac{\partial u}{\partial t}$ ) denotes the derivative of $u$ with respect to its first (resp. second) variable and $\mathbb{P}$ stands for the set of positive real numbers.

These equations have been studied by many different means ( [16]- [51], [118], [77]...).

In the present paper we survey the use of tools from nonsmooth analysis and from duality theory to find explicit solutions of (1)-(2). We partially follow the stream of the papers [18]- [25] culminating in [4]. When preparing [127], [128], [94], [104] we were not aware of some of the works presenting bridges between the study of partial differential equations and nonsmooth analysis ( [13], [27], [35], [48], [54]- [57], [77], [78], [122]...). It appears that the two fields have more links than expected. In particular, the nonlinear character of equation (1) leads to use test functions in a manner which differs from the one used in the theory of distributions: instead of taking the duality product of $u$ with a smooth test function $\varphi$, one picks a smooth function $\varphi$ which minorizes $u$. The new use is almost equivalent to the use of elementary subdifferentials; moreover, it appears that several proofs in the studies of Hamilton-Jacobi equations have similarities with the proofs of fuzzy sum rules in nonsmooth analysis, which ensure decoupling via a penalization method.

Many problems of applied mathematics can be solved because one uses useful transforms as a tool. Among these transforms are the Fourier transform, the Laplace transform, the Radon and Cramer transforms. The benefit of such tools consists in replacing a problem by a simpler one in some dual space, and then in coming back to the initial problem. In several instances, some complex operations (such as derivations, convolutions) are transformed into simpler ones. Duality techniques have been in use in the field of Hamilton-Jacobi equations for a long time (see for instance [60], [76], [51]...); however only recently a systematic use of duality methods has been developed ( [4], [18]- [20], [61], [94], [104], [127], [128]). These methods shed new light on various classical results. Here we consider both convex duality (via the Legendre-Fenchel transform) and quasiconvex dualities and we stress the analogies between the two cases. We also present new results as we consider dualities which have not been used yet and as we make rather weak semicontinuity assumptions. In particular, we accept Hamiltonians and initial value functions which may take the value $+\infty$ (and even the value $-\infty$ !) or are discontinuous. Some care is required because the transforms $f \mapsto f^{\sharp}$ and $f \mapsto f^{\%}$ extensively used in [4], [18]- [20] are not dualities. Thus we give a detailed presentation of such transforms in a preliminary section.

We also stress the uses of different notions of "viscosity" solutions; they are related to notions of nonsmooth analysis. We choose to separate assumptions for (1) from assumptions which ensure (2); of course, the conjunction of the assumptions give conditions to solve the system (1)-(2). But our choice enables to detect subsolutions and supersolutions in situations which would be excluded by the union of the two sets of assumptions; along with comparison theorems such as [13, Theorem 3.7 p. 56], [16, p. 99], one can get in this way estimates about solutions. We use variational convergence in a systematic way to deal with initial conditions, while most approaches treat them in a classical way, omitting upper epiconvergence or Mosco convergence (however, see [17], [119]). Another apparently new feature of our approach is that we use functions which are related to $g$ and $H$ and their conjugates, but not necessarily themselves, in order to find supersolutions and subsolutions. In this way we get a flexibility the usual explicit formulas do not offer; moreover we get a means to obtain comparison results since we can substitute to $\mathrm{g}$ and $H$ other functions for which the computations are simpler.

Also, it is our purpose to convince the reader that a parallel development of the convex case (in which $H$ is supposed to be independent of its scalar variable) and of the quasiconvex case is a fruitful approach. For this reason, we display these two cases in a close manner, according to the following table of contents:

1. Introduction

2. Preliminaries: dualities

3. Some notions of solution

4. The Hopf-Lax type formula in the convex case

5. The Hopf formulas in the quasiconvex case

6. The Lax-Oleinik solution in the convex case

7. The Lax-Oleinik formulas in the quasiconvex case

8. Initial conditions

9. Comparison and uniqueness results 
10. Stability results

11. Coincidence of the Hopf and of the Lax solutions

12. Conclusion

\section{Preliminaries: Dualities}

We will use various notions of conjugate function. Throughout, for any function $f$ on $X \times \mathbb{R}$, we denote by $f^{*}$ its convex (or Legendre-Fenchel) conjugate with respect to the state variable $x \in X$ :

$$
f^{*}(p, q):=f_{q}^{*}(p):=\sup _{x \in X}(\langle p, x\rangle-f(x, q)) \quad \text { for }(p, q) \in X^{*} \times \mathbb{R}
$$

where $\langle p, x\rangle$, also denoted by $p . x$, denotes the value of $p \in X^{*}$ on $x \in X$ and $f_{q}:=f(\cdot, q)$. We use the same notation for a function $f$ on $X$, considering it as a function on $X \times \mathbb{R}$ which is independent of its second variable. When $f$ is defined on $X^{*} \times \mathbb{R}$ we do the same.

When $f: X \rightarrow \overline{\mathbb{R}}$ is l.s.c. and quasiconvex, we note that in order to recover the function through biconjugacy, one defines its conjugate on $Y=X^{*} \times \mathbb{R}$ rather than on $X^{*}$. Using just $X^{*}$ would not suffice in general. Moreover, since quasiconvexity of $f$ is a property of the sublevel sets of $f$, it is natural to assign a key role to these sublevel sets. Several devices are known. Here we just consider two groups of correspondences. Namely, we set, for $f: X \rightarrow \overline{\mathbb{R}}, y:=(p, q) \in Y$,

$$
\begin{aligned}
f^{b}(p, q) & :=\sup \{p \cdot x: x \in[f<q]\}, \\
f^{\sharp}(p, q) & :=\sup \{p \cdot x: x \in[f \leq q]\}, \\
f^{\div}(p, q) & :=\sup \{p \cdot x-f(x): x \in[f<q]\}, \\
f^{\%}(p, q) & :=\sup \{p \cdot x-f(x): x \in[f \leq q]\} .
\end{aligned}
$$

The conjugates $f^{\sharp}, f^{\%}$ have also been considered in [4] and [18]- [23], but the use of $f^{\div}, f^{b}$ seems to be new. The assertions of the following lemma are easy consequences of the definitions. In the sequel it will be convenient to say that a function $h: X^{*} \rightarrow \overline{\mathbb{R}}$ is positively homogeneous if either it is identically $-\infty$ or if $h(0)=0$ and if $h(r p)=r h(p)$ for every $p \in X, r \in \mathbb{P}$. If moreover $h$ is convex we say that $h$ is sublinear.

Lemma 2.1. (a) These four conjugates are nondecreasing functions of their second variables and l.s.c. convex functions of their first variables. Moreover, for fixed $q$, the functions $f^{b}(\cdot, q), f^{\sharp}(\cdot, q), f^{\div}(\cdot, q)$ and $f^{\%}(\cdot, q)$ are either proper l.s.c. convex functions or are identically equal to $-\infty$.

(b) If $[f<q]$ (resp. if $[f \leq q]$ ) is nonempty, then $f^{b}(0, q)=0$ (resp. $\left.f^{\sharp}(\cdot, q)=0\right)$.

(c) $f^{b}$ and $f^{\sharp}$ are l.s.c. sublinear functions of their first variables.

(d) $f^{b}$ and $f \div$ are jointly l.s.c. in $(p, q)$.

(e) If $f$ has no local minimizer, then $f^{b}=f^{\sharp}$ and $f^{\div}=f^{\%}$.

It follows from assertion (d) or from the relations $[f<q]=\bigcup_{r<q}[f<r]=\bigcup_{r<q}[f \leq r]$ for $q \in \mathbb{R}$, that for every $(p, q) \in X^{*} \times \mathbb{R}$ one has

$$
\begin{aligned}
f^{b}(p, q) & =\sup _{r<q} f^{b}(p, r)=\sup _{r<q} f^{\sharp}(p, r), \\
f^{\doteqdot}(p, q) & =\sup _{r<q} f^{\doteqdot}(p, r)=\sup _{r<q} f^{\%}(p, r) .
\end{aligned}
$$

Another link between these correspondences is revealed by the relations of the following lemma. 
Lemma 2.2. For any function $f$ on $X$ one has

$$
\begin{aligned}
& f^{\div}(p, q)=\sup _{r<q}\left(f^{b}(p, r)-r\right)=\sup _{r<q}\left(f^{\sharp}(p, r)-r\right), \\
& f^{\%}(p, q)=\sup _{r \leq q}\left(f^{\sharp}(p, r)-r\right) .
\end{aligned}
$$

ProOF. Let us check the first relation, the proof of the last one being similar. Obviously, for any $(p, q) \in$ $X^{*} \times \mathbb{R}$ and any $s<\sup _{r<q}\left(f^{\sharp}(p, r)-r\right)$ one can find $r<q$ and $x \in[f \leq r]$ such that $s<p . x-r$; thus $s<p . x-f(x) \leq f^{\div}(p, q)$ and so $\sup _{r<q}\left(f^{b}(p, r)-r\right) \leq \sup _{r<q}\left(f^{\sharp}(p, r)-r\right) \leq f \div(p, q)$. On the other hand, for every $t<f \div(p, q)$ one can find some $x \in[f<q]$ such that $t<p . x-f(x)$. Taking $r \in] f(x), q[$ such that $r<p . x-t$ we get $t<p . x-r \leq f^{b}(p, r)-r$. Thus $\sup _{r<q}\left(f^{\sharp}(p, r)-r\right) \geq \sup _{r<q}\left(f^{b}(p, r)-r\right) \geq f^{\div}(p, q)$ and equalities hold.

The four formulas preceding Lemma 2.1 define quasi-dualities in the sense of [94] that each of these operations $f \mapsto f^{D}$ can be determined by a generating function $G_{D}: X \times Y \times \overline{\mathbb{R}} \rightarrow \overline{\mathbb{R}}$ which is nonincreasing in its third variable through the representation

$$
f^{D}(y)=\sup _{x \in X} G_{D}(x, y, f(x)) \quad \text { for } y=(p, q) \in Y .
$$

Introducing the indicator function $\iota_{S}$ of a subset $S$ of a space $T$ as the function given by $\iota_{S}(t)=0$ for $t \in S$, $\iota_{S}(t)=+\infty$ for $t \in T \backslash S$, the generating functions associated with the considered quasi-dualities can be given explicitly by

$$
\begin{aligned}
G_{b}(x, p, q, r) & =p \cdot x-\iota_{[-\infty, q[}(r), \quad G_{\sharp}(x, p, q, r)=p . x-\iota_{[-\infty, q]}(r), \\
G_{\div}(x, p, q, r) & =p \cdot x-r-\iota_{[-\infty, q[}(r), \quad G_{\%}(x, p, q, r)=p . x-r-\iota_{[-\infty, q]}(r) .
\end{aligned}
$$

The extension presented in [94] to quasi-dualities of the process of [81] enables one to give a reverse correspondence, also denoted by $F \mapsto F^{D}$ (or $F \mapsto F^{D^{\prime}}$ if some confusion may arise), from $\overline{\mathbb{R}}^{Y}$ to $\overline{\mathbb{R}}^{X}$, given by $F^{D}(x)=\sup _{y \in Y} G_{D}^{\prime}(y, x, F(y))$ for $F \in \overline{\mathbb{R}}^{Y}$, where

$$
G_{D}^{\prime}(y, x, s):=\inf \left\{r \in \overline{\mathbb{R}}: G_{D}(x, y, r) \leq s\right\}
$$

Note that for every $(x, y) \in X \times Y$ the function $G_{D}^{\prime}(y, x,-\cdot)$ is obtained as the upper quasi-inverse of $-G_{D}(x, y, \cdot)$ in the sense of [102]. In the case we have presented, these quasi-inverses can be explicitely computed: $G_{b}^{\prime}((p, q), x, s)=G_{\sharp}^{\prime}((p, q), x, s)=q-\iota_{[-\infty, 0[}(s-p . x)$, and $G_{\%}^{\prime}((p, q), x, s)=G_{\div}^{\prime}((p, q), x, s)=q \wedge(p . x-s)$ (see [94] Examples 7.6 and 7.7$)$ and $\left(G_{b}^{\prime}\right)^{\prime}=G_{b},\left(G_{\div}^{\prime}\right)^{\prime}=G_{\div}$.

The transform $F \mapsto F^{D}$ is then a duality and for any $f \in \overline{\mathbb{R}}^{X}$ one has $f^{D D}:=\left(f^{D}\right)^{D} \leq f([94$, Lemma 3.4]). Since $G_{D}$ is nonincreasing in its third variable, for any $F \in \overline{\mathbb{R}}^{Y}$ one has

$$
\begin{aligned}
F^{D}(x) & =\sup _{y \in Y} \sup \left\{r \in \overline{\mathbb{R}}: G_{D}(x, y, r)>F(y)\right\} \\
& =\sup \left\{r \in \overline{\mathbb{R}}: \exists y \in Y, G_{D}(x, y, r)>F(y)\right\}
\end{aligned}
$$


In the case of the flat duality, setting $F_{q}:=F(\cdot, q), F_{r+0}:=\inf _{q>r} F_{q}$, so that $F_{r+0}^{*}:=\left(F_{r+0}\right)^{*}=\sup _{q>r} F_{q}^{*}$, we get

$$
\begin{aligned}
F^{b}(x) & =\sup \{r \in \overline{\mathbb{R}}: \exists(p, q) \in Y, q>r, p . x>F(p, q)\} \\
& =\sup \left\{r \in \overline{\mathbb{R}}: \exists q>r, F_{q}^{*}(x)>0\right\} \\
& =\sup \left\{r \in \overline{\mathbb{R}}: \sup _{q>r} F_{q}^{*}(x)>0\right\} \\
& =\sup \left\{r \in \overline{\mathbb{R}}: F_{r+0}^{*}(x)>0\right\} .
\end{aligned}
$$

Hence

$$
\begin{gathered}
s<F^{b}(x) \Leftrightarrow\left(\exists q>s: F_{q}^{*}(x)>0\right), \\
F^{b}(x) \leq r \Leftrightarrow\left(\forall q>r: F_{q}^{*}(x) \leq 0\right) \Leftrightarrow F_{r+0}^{*}(x) \leq 0
\end{gathered}
$$

or, since a similar computation shows that the sharp conjugate of $F$ is given by the same relations

$$
F^{b}(x) \leq q \Longleftrightarrow F^{\sharp}(x) \leq q \Longleftrightarrow x \in \bigcap_{r>q}\left[F_{r}^{*} \leq 0\right]=\left[F_{q+0}^{*} \leq 0\right] .
$$

In particular, it ensues that we have the following result, a converse of which will be given in Proposition 2.5.

Lemma 2.3. For any function $F: X^{*} \times \mathbb{R} \rightarrow \overline{\mathbb{R}}$, the function $F^{b}=F^{\sharp}$ is quasiconvex and l.s.c.

While the definitions of the transforms $f \mapsto f^{\sharp}$ and $f \mapsto f^{b}$ are natural for quasiconvex functions since they involve the sublevel sets of the function, the definition of the reverse duality $F \mapsto F^{b}=F^{\sharp}$ may appear as rather involved. However, we observe that the function $F^{b}=F^{\sharp}$ is characterized by its sublevel sets, a classical way of defining a function (see [124], for example).

The conjugate functions $F^{\%}$ and $F^{\doteqdot}$ of $F$ associated with $G_{\%}$ and $G_{\div}$also coincide. They are given by

$$
F^{\div}(x)=F^{\%}(x):=\sup _{q \in \mathbb{R}} F_{q}^{*}(x) \wedge q .
$$

This formula makes clear that the inequality $F^{\div}(x) \leq r$ is equivalent to $F_{q}^{*}(x) \leq r$ for any $q>r$; moreover, taking into account the relation $F_{r+0}^{*}:=\left(\inf _{s>r} F_{s}\right)^{*}=\sup _{s>r} F_{s}^{*}$, we get the following lemma.

Lemma 2.4. For any function $F: X^{*} \times \mathbb{R} \rightarrow \overline{\mathbb{R}}$, the function $F^{\div}=F^{\%}$ is quasiconvex and l.s.c. and for any $r \in \mathbb{R}$ one has, with $F_{r+0}:=\inf _{s>r} F_{s}$,

$$
\left[F^{\div} \leq r\right]=\left[F^{\%} \leq r\right]=\bigcap_{s>r}\left[F_{s}^{*} \leq r\right]=\left[F_{r+0}^{*} \leq r\right] .
$$

This conjugate function belongs to an important subclass of the class of quasiconvex functions we suggest to call the class of truncavex functions on $X$; here we say that a function is a truncavex function if it is a supremum of a family of truncated continuous affine functions, i.e. a supremum of a family of functions of the form $a(\cdot) \wedge q$ where $a(\cdot)$ is a continuous affine function on $X$ and $q$ is a constant. This class of functions has interesting duality properties (see [79], [100]). Let us note that this class of functions is stable by truncation since for any family $\left(a_{i}\right)_{i \in I}$ of affine functions and any family $\left(q_{i}\right)_{i \in I}$ of real numbers one has for $q \in \mathbb{R}$

$$
\left(\sup _{i \in I} a_{i} \wedge q_{i}\right) \wedge q=\sup _{i \in I}\left(a_{i} \wedge\left(q_{i} \wedge q\right)\right) .
$$


The fact that $F^{\%}=F^{\div}$belongs to the class of truncavex functions stems from (4) and the following relations in which, for $q \in \mathbb{R}, E(q)$ is the epigraph of $F_{q}$ and $E$ is the union over $q \in \mathbb{R}$ of the sets $E(q)$

$$
F^{\%}(x)=\sup _{q \in \mathbb{R}}\left[\left(\sup _{(p, r) \in E(q)}(p \cdot x-r)\right) \wedge q\right]=\sup _{q \in \mathbb{R}}\left[\sup _{(p, r) \in E(q)}(p \cdot x-r) \wedge q\right]=\sup _{(p, q) \in E}\left(p . x-F_{q}(p)\right) \wedge q .
$$

Let us give some more attention to the quasi-dualities described above.

Lemma 2.5. (a) The mappings $D: f \longmapsto f^{b}$ and $f \longmapsto f \div$ are dualities, i.e. satisfy

$$
D\left(\inf _{i \in I} f_{i}\right)=\sup _{i \in I} D\left(f_{i}\right)
$$

(b) The four correspondences defined above are hemi-dualities in the sense that for any function $f \in \overline{\mathbb{R}}^{X}$ one has

$$
f^{b b} \leq f, \quad f^{\sharp \sharp} \leq f, \quad f \div \div \leq f, \quad f^{\% \%} \leq f .
$$

(c) The mappings $F \longmapsto F^{b}=F^{\sharp}$ and $F \longmapsto F^{\div}=F^{\%}$ are dualities.

Proof. (a) The assertion follows from the fact that the generating functions $G_{D}$ associated with the quasidualities $f \longmapsto f^{b}$ and $f \longmapsto f^{\div}$satisfy $G_{D}\left(x, p, q, \inf _{i \in I} r_{i}\right)=\sup _{i \in I} G_{D}\left(x, p, q, r_{i}\right)$.

(b) The assertion is a consequence of the inequality $f^{D D} \leq f$ mentioned above since $f \longmapsto f^{D}$ is a quasiduality. Alternatively, since a duality is an hemi-duality ( $[94])$, only the cases of $f \longmapsto f^{\sharp}$ and $f \longmapsto f^{\%}$ have to be considered. For each $x \in X$ and each $p \in X^{*}$ we have $p . x \leq f^{\sharp}(p, r)$ for $r=f(x)$, hence $\left(f_{r}^{\sharp}\right)^{*}(x) \leq 0$ and $f^{\sharp \sharp}(x) \leq f(x)$. Here we use the fact that if $F$ is nondecreasing in its second variable (and this is the case for $\left.F:=f^{\sharp}\right)$, one has $F^{\sharp}(x) \leq r$ whenever $\left(F_{r}\right)^{*}(x) \leq 0$. For $(p, q) \in X^{*} \times \mathbb{R}, q \geq r:=f(x)$ one has $f^{\%}(p, q) \geq p . x-r$, hence $\left(f_{q}^{\%}\right)^{*}(x) \leq r, q \wedge\left(f_{q}^{\%}\right)^{*}(x) \leq r$ and, of course, $q \wedge\left(f_{q}^{\%}\right)^{*}(x) \leq r$ for $q<r$, so that $\sup _{q \in \mathbb{R}} q \wedge F_{q}^{*}(x) \leq r$ for $F:=f^{\%}$.

(c) The assertion is a general result about the reverse duality associated with a quasi-duality ( $[94$, Lemma $3.4]$ ); let us give a direct proof for the reader's convenience. Given a family $\left(F_{i}\right)_{i \in I}$ of functions on $X^{*} \times \mathbb{R}$, let $F:=\inf _{i \in I} F^{i}$. Then, for any $q \in \mathbb{R}$ one has $F_{q+0}^{*}=\left(\inf _{r>q, i \in I} F_{r}^{i}\right)^{*}=\sup _{i \in I} \sup _{q>r}\left(F_{r}^{i}\right)^{*}$, so that for every $x \in X$ one has $F^{b}(x) \leq q$ (resp. $\left.F^{\%}(x) \leq q\right)$ iff for every $i \in I$ one has $\left(F^{i}\right)^{b}(x) \leq q\left(\operatorname{resp} .\left(F^{i}\right)^{\%}(x) \leq q\right)$ hence $F^{b}=\sup _{i \in I}\left(F^{i}\right)^{b}\left(\operatorname{resp} . F^{\%}=\sup _{i \in I}\left(F^{i}\right)^{\%}\right)$.

It can be shown that $f \longmapsto f^{b}$ is the duality associated to $F \longmapsto F^{b}$ in the sense of [94, Lemma 3.2], i.e.

$$
f^{b}=\inf \left\{F \in \overline{\mathbb{R}}^{X^{*} \times \mathbb{R}}: F^{b} \leq f\right\}
$$

In fact, let $G:=\inf \left\{F \in \overline{\mathbb{R}}^{X^{*} \times \mathbb{R}}: F^{b} \leq f\right\}$. Since $\left(f^{b}\right)^{b}=f^{b b} \leq f$, one has $G \leq f^{b}$; on the other hand, for every $F$ satisfying $F^{b} \leq f$ one has $F \geq F^{b b} \geq f^{b}$, hence $G \geq f^{b}$. Thus $G=f^{b}$. Although the two quasi-dualities $f \longmapsto f^{\sharp}$ and $f \longmapsto f^{\%}$ have been used intensively in [4], [18]- [20], they are not dualities. Therefore, their use requires some care; in particular, for $F \in \overline{\mathbb{R}}^{Y}$, the inequalities $F^{\sharp \sharp} \leq F, F^{\%} \% \leq F$ are not always satisfied.

Example 1. Let $F: X^{*} \times \mathbb{R} \rightarrow \overline{\mathbb{R}}$ be given by $F(p, q)=-\infty$ for $\left.\left.(p, q) \in X^{*} \times\right]-\infty, 0\right], F(p, q)=\|p\|$ for $(p, q) \in X \times] 0,1], F(p, q)=+\infty$ for $\left.(p, q) \in X^{*} \times\right] 1,+\infty\left[\right.$. Then $F^{\sharp}$ is the quasiconvex function $1-\chi_{B}$, where $\chi_{B}$ denotes the characteristic function of the closed unit ball $B$ of $X$ (i.e. $\chi_{B}(x)=1$ for $x \in B, 0$ else) and, for $p \neq 0, F^{\sharp \sharp}(p, 1)=+\infty>F(p, 1)=\|p\|$.

The fact that $f \mapsto f^{\sharp}$ is not a duality is illustrated by the next example. 
Example 2. Let $f: \mathbb{R} \rightarrow \mathbb{R}$ be given by $f(x)=0$ for $x \in]-\infty, 1[, f(x)=1$ for $x \in[1,+\infty[$ and let $f_{n}=c_{n} f$ where $\left(c_{n}\right) \rightarrow 1, c_{n}>1$ for each $n$. Then $f^{\sharp}(1,1)=+\infty, f_{n}^{\sharp}(1,1)=1$ for each $n \geq 0, p>0$, so that $\left(\inf _{n} f_{n}\right)^{\sharp} \neq \sup _{n} f_{n}^{\sharp}$.

The properties of the conjugate of a function $F$ on $X^{*} \times \mathbb{R}$ given in the following statement will be used later on. We recall that, for $(p, q) \in X^{*} \times \mathbb{R}$,

$$
F_{q+0}(p):=F(p, q+0):=\inf _{r>q} F(p, r) .
$$

When $F(p, \cdot)$ is nondecreasing, one has $F(p, q+0):=\lim _{r \rightarrow q_{+}} F(p, r)$, what justifies the notation. We notice that the conjugate $h^{*}: X \rightarrow \overline{\mathbb{R}}$ of a positively homogeneous function $h$ is the indicator function of (the possibly empty) set $\partial^{c} h(0):=\left\{x \in X: \forall p \in X^{*} p \cdot x \leq h(p)\right\}$ since $t h^{*}=\left(h_{t}\right)^{*}=h^{*}$ for every $t>0$, where $h_{t}(p)=t h(p / t)$.

Lemma 2.6. (a) For any function $F: X^{*} \times \mathbb{R} \rightarrow \overline{\mathbb{R}}$ and for any $r \in \mathbb{R}$, one has

$$
\begin{aligned}
{\left[F^{b} \leq r\right] } & =\left[F^{\sharp} \leq r\right]=\left[F_{r+0}^{*} \leq 0\right], \\
F^{\sharp}(x) & =F^{b}(x)=\inf \left\{r \in \mathbb{R}: F_{r+0}^{*}(x) \leq 0\right\}
\end{aligned}
$$

and $F^{\sharp \sharp}(\cdot, r) \leq F(\cdot, r+0)$. If for every $r \in \mathbb{R}$ either $F(0, r)=0$ or $F(p, r)=-\infty$ for every $p \in X^{*}$, one also has $\left[F^{\sharp} \leq r\right]=\partial^{c} F_{r+0}(0)$.

(b) If for every $p \in X^{*}$ the function $F(p, \cdot)$ is nondecreasing then, for every $x \in X$ one has $F^{b}(x)=F^{\sharp}(x)=$ $\inf \left\{q \in \mathbb{R}: F_{q}^{*}(x) \leq 0\right\}$.

(c) When for each $p \in X^{*}$ the function $F(p, \cdot)$ is u.s.c. and nondecreasing, one has $F^{\sharp} \leq F$ and

$$
F^{b}(x)=F^{\sharp}(x)=\min \left\{q \in \mathbb{R}: F_{q}^{*}(x) \leq 0\right\},
$$

in the usual sense that the infimum is attained when it is finite. If moreover for each $q \in \mathbb{R}$ the function $F_{q}:=F(\cdot, q)$ is sublinear and weak $k^{*}$ l.s.c., one has $F^{\sharp \sharp}=F$.

A more refined criteria for the equality $F^{\sharp \sharp}=F$ will be given in Proposition 2.16 below.

Proof. (a) Relation (7) is just a rewriting of relation (3). Relation (8) stems from the fact that $q \mapsto F_{q+0}$ is nondecreasing, so that $q \mapsto F_{q+0}^{*}$ is nonincreasing. Given $r \in \mathbb{R}, p \in X^{*}$, let $q<F^{\sharp \sharp}(p, r)$. Then there exists some $x \in\left[F^{\sharp} \leq r\right]$ such that $q<p . x$. Relation (7) ensures that $F_{r+0}^{*}(x) \leq 0$, hence $p . x \leq F(p, r+0)$ and $q<F(p, r+0)$. Therefore $F^{\sharp \sharp}(p, r) \leq F(p, r+0)$. In order to prove the last assertion of (a), for a given $r \in \mathbb{R}$, let us set $h:=F_{r+0}$. If $h(0)=0$, the relation $h^{*}(x) \leq 0$ means that $p . x \leq h(p)$ for every $p \in X^{*}$ or that $x \in \partial^{c} h(0)$. Thus $F^{\sharp}(x) \leq r$ means that $x \in \partial F_{r+0}(0)$. If $h(0)=-\infty$, then, for every $x \in X$, one has $h^{*}(x)=+\infty$ and $x \notin\left[F^{\sharp} \leq r\right]$; thus $\left[F^{\sharp} \leq r\right]=\varnothing=\partial^{c} F_{r+0}(0)$.

(b) When for every $p \in X^{*}$ the function $F(p, \cdot)$ is nondecreasing, then for every $x \in X$ the function $q \mapsto F_{q}^{*}(x)$ is nonincreasing, so that $F_{r+0}^{*}(x)=\sup _{s>r} F_{s}^{*}(x) \leq F_{r}^{*}(x)$ and

$$
s:=\inf \left\{r \in \mathbb{R}: F_{r+0}^{*}(x) \leq 0\right\} \leq \inf \left\{q \in \mathbb{R}: F_{q}^{*}(x) \leq 0\right\} .
$$

On the other hand, for any $t \in \mathbb{R}$ with $t>s$ one can find $r \in] s, t\left[\right.$ such that $F_{r+0}^{*}(x) \leq 0$. Thus $F_{t}^{*}(x) \leq 0$, hence $\inf \left\{q \in \mathbb{R}: F_{q}^{*}(x) \leq 0\right\} \leq s$ and equality holds.

(c) When for each $p \in X^{*}$ the function $F(p, \cdot)$ is u.s.c. and nondecreasing, one has $F_{q+0}=F_{q}$ for every $q \in \mathbb{R}$. Thus $F^{\sharp \sharp} \leq F$ by (a). Suppose $r:=\inf \left\{q \in \mathbb{R}: F_{q}^{*}(x) \leq 0\right\}$ is finite. Then there exists a sequence $\left(r_{n}\right) \rightarrow r$ such that $p . x-F\left(p, r_{n}\right) \leq 0$ for all $p \in X^{*}$. Since $F(p, \cdot)$ is u.s.c., we get $p . x \leq F(p, r)$ for all $p \in X^{*}$ or $F_{r}^{*}(x) \leq 0$. The last assertion is a consequence of $(7)$ : when $F_{q}$ is sublinear and weak* ${ }^{*}$.s.c., $F_{q}$ is the supremum 
of the family of weak* continuous linear forms $x$ majorized by $F_{q}$ i.e. in $\left[F_{q}^{*} \leq 0\right]$; thus for every $p \in X^{*}$, since $\left[F^{\sharp} \leq q\right]=\left[F_{q}^{*} \leq 0\right]$, one has

$$
F^{\sharp \sharp}(p, q)=\sup \left\{p \cdot x: x \in\left[F_{q}^{*} \leq 0\right]\right\}=F_{q}(p) .
$$

Remark. Since the sublevel sets $\left[F^{b} \leq q\right]$ of $F^{b}$ are determined by inequalities of the type $k^{*}(x) \leq 0$ for some function $k:=F_{q+0}$ on $X^{*}$, we must focus our attention on the set $K$ determined by such an inequality. We notice that for every $t>0$, the set $K$ is also determined by the inequality $t k^{*}(x) \leq 0$, i.e. $\left(k_{t}\right)^{*}(x) \leq 0$, where

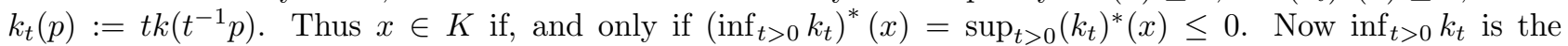
positively homogeneous hull of $k$, i.e. the greatest positively homogeneous function majorized by $k$. Moreover, since the conjugate of a function $h$ is equal to the conjugate of its convex hull, or even to the conjugate of $h^{* *}$, we obtain that $F^{b}$ coincides with $(\widehat{F})^{b}$, where, for each $q \in \mathbb{R}, \widehat{F}_{q}:=\widehat{F(\cdot, q)}$ is the l.s.c. sublinear hull of $F_{q}:=F(\cdot, q)$.

It is known that a function $f: X \rightarrow \overline{\mathbb{R}}$ is l.s.c. and quasiconvex iff $f=f^{\text {bb }}$ iff $f=f^{\sharp \sharp}$ (see [4], [128] Theorem 4, [94] and Proposition 2.8 below). We deduce this well known result ( [42]- [44]) from the following characterization of the l.s.c. quasiconvex hull $h$ of a function $f$, which is the greatest l.s.c. quasiconvex function majorized by $f$. A more direct proof is presented in [105]. Here $\overline{c o} A$ denotes the closed convex hull of a subset $A$ of $X$.

Lemma 2.7. For any $f: X \rightarrow \overline{\mathbb{R}}$, the l.s.c. quasiconvex hull $h$ of $f$ is characterized by $[h \leq q]=\bigcap_{r>q} \overline{c o}[f \leq$ $r]=\bigcap_{r>q} \overline{c o}[f<r]$.

Proof. The function $h$ given by

$$
h(x):=\inf \{r \in \mathbb{R}: x \in \overline{c o}[f \leq r]\}
$$

satisfies $[h \leq q]=\bigcap_{r>q} \overline{c o}[f<r] \supset[f \leq q]$, hence is l.s.c. quasiconvex and $h \leq f$. If $g$ is a l.s.c. quasiconvex function majorized by $f$, for each $r>q$ we have $[f<r] \subset[f \leq r] \subset[g \leq r]$, hence

$$
\bigcap_{r>q} \overline{c o}[f<r] \subset \bigcap_{r>q} \overline{c o}[f \leq r] \subset \bigcap_{r>q}[g \leq r]=[g \leq q]
$$

hence $g \leq h$. Therefore $h$ is indeed the l.s.c. quasiconvex hull $h$ of $f$.

Proposition 2.8. For any $f: X \rightarrow \overline{\mathbb{R}}$, the l.s.c. quasiconvex hull of $f$ is $f^{b b}=f^{\sharp \sharp}$. In particular $f$ is l.s.c. quasiconvex iff $f=f^{\text {bb }}$ iff $f=f^{\sharp \#}$.

Proof. Let $F(p, q):=f^{b}(p, q)=\iota_{[f<q]}^{*}(p)$, where $\iota_{S}$ is the indicator function of the subset $S$ of $X$. We observe that for each $x \in X$ and each $r \in \mathbb{R}$ we have

$$
F_{r}^{*}(x)=\iota_{[f<r]}^{* *}(x)=\iota_{\overline{c o}[f<r]}(x)
$$

Therefore, if $h$ denotes the l.s.c. quasiconvex hull of $f$, we have

$$
h(x) \leq q \Leftrightarrow x \in \bigcap_{r>q} \overline{c o}[f<r] \Leftrightarrow \forall r>q \quad F_{r}^{*}(x) \leq 0 \Leftrightarrow f^{b b}(x) \leq q .
$$

Thus $h=f^{b b}$. The proof with $F=f^{\sharp}$ is similar since $[h \leq q]=\bigcap_{r>q} \overline{c o}[f \leq r]$.

For completeness, let us consider the case of the other two transforms, which has been dealt with in [4, Lemma 4.4], [79, Cor. 4.3] and [100, Thm 1.8]. 
Proposition 2.9. For any $f: X \rightarrow \overline{\mathbb{R}}$, the truncavex hull of $f$ is $f^{\div \div}=f^{\% \%}$. In particular $f$ is truncavex iff $f=f \div \div$ iff $f=f^{\% \%}$.

Proof. When $f=f^{\div} \div$or $f=f^{\% \%}, f$ is clearly truncavex. Let us prove the converse. Since $f^{\div} \leq f^{\sharp}$, we always have $f \geq f^{\div \div} \geq f^{\% \%}$. Thus, it suffices to show that when $f$ is truncavex one has $f(x) \leq f^{\% \%}(x)$ for every $x \in X$. Let $r \in \mathbb{R}$ be such that $r \geq f^{\% \%}(x)$ and let us prove that $f(x) \leq r$. The inequality $f(x) \leq r$ being obvious when $r \geq \sup f$, we may suppose $r<\sup f$. By definition of $f^{\% \%}$, for every $\left.s \in\right] r$, sup $f[$ we have $\left(f_{s}^{\%}\right)^{*}(x) \leq r$ and we can find $a \in X^{*}$ and $b \in \mathbb{R}$ such that $a+b+\iota_{[f \leq s]} \leq f+\iota_{[f \leq s]}$ hence, since $[f \leq s]$ is closed convex,

$$
a . x+b+\iota_{[f \leq s]}(x) \leq\left(f+\iota_{[f \leq s]}\right)^{* *}(x)=\left(f_{s}^{\%}\right)^{*}(x) \leq r .
$$

Thus $x \in[f \leq s]$ for every $s \in] r$, sup $f$. Therefore $f(x) \leq r$. Now if $f$ is an arbitrary function, for any truncavex function $g \leq f$ we have $g \div \geq f \div$ and $g=g \div \div \leq f \div \div$. Thus $f \div \div$ is the greatest truncavex function majorized by $f$.

Lemma 2.10. (a) For any function $F: X^{*} \times \mathbb{R} \rightarrow \overline{\mathbb{R}}$ and for any $r \in \mathbb{R}$, one has

$$
\begin{aligned}
{\left[F^{\div} \leq r\right] } & =\left[F^{\%} \leq r\right]=\left[F_{r+0}^{*} \leq r\right], \\
F^{\%}(x) & =F^{\div}(x)=\inf \left\{r \in \mathbb{R}: F_{r+0}^{*}(x) \leq r\right\}
\end{aligned}
$$

and $F^{\div \div}(\cdot, r) \leq F^{\% \%}(\cdot, r) \leq F(\cdot, r+0)$. (b) If for every $p \in X^{*}$ the function $F(p, \cdot)$ is nondecreasing then, for every $x \in X$, one has $F^{\div}(x)=F^{\%}(x)=\inf \left\{q \in \mathbb{R}: F_{q}^{*}(x) \leq q\right\}$.

(c) When for each $p \in X^{*}$ the function $F(p, \cdot)$ is u.s.c. and nondecreasing, one has $F^{\div \div} \leq F^{\%} \% \leq$ and

$$
F^{\div}(x)=F^{\%}(x)=\min \left\{q \in \mathbb{R}: F_{q}^{*}(x) \leq q\right\}
$$

in the usual sense that the infimum is attained when it is finite.

Proof. (a) Relation (10) is just a rewriting of relation (5). Relation (11) ensues. Given $r \in \mathbb{R}, p \in X^{*}$, let show that $F^{\% \%}(p, r) \leq F(p, r+0)$, or, equivalently, that for any $x \in X$ such that $F^{\%}(x) \leq r$ and any $s>r$ one has $p . x-F^{\%}(x) \leq F(p, s)$. Now, for any $t \in\left[F^{\%}(x), r\right] \cap \mathbb{R}$ we have $F_{s}^{*}(x) \leq t$ since $s>t$, hence $p . x-F(p, s) \leq t$. Taking the infimum over $t \in\left[F^{\%}(x), r\right] \cap \mathbb{R}$ in this inequality, we get $p . x-F^{\%}(x) \leq F(p, s)$, as expected.

(b) When for every $p \in X^{*}$ the function $F(p, \cdot)$ is nondecreasing, one has $F_{r} \leq F_{r+0}$, hence $F_{r+0}^{*} \leq F_{r}^{*}$ and, for every $x \in X$

$$
s:=\inf \left\{r \in \mathbb{R}: F_{r+0}^{*}(x) \leq r\right\} \leq \inf \left\{q \in \mathbb{R}: F_{q}^{*}(x) \leq q\right\} .
$$

On the other hand, for any $t \in \mathbb{R}$ with $t>s$ one can find $r \in] s, t\left[\right.$ such that $F_{r+0}^{*}(x) \leq r$. Thus $F_{t}^{*}(x) \leq$ $F_{r+0}^{*}(x) \leq r<t$, hence inf $\left\{q \in \mathbb{R}: F_{q}^{*}(x) \leq q\right\} \leq s$ and equality holds in (12).

(c) When for each $p \in X^{*}$ the function $F(p, \cdot)$ is u.s.c. and nondecreasing, one has $F_{q+0}=F_{q}$ for every $q \in \mathbb{R}$, so that, by (a) $F^{\% \%} \leq F$. Moreover, in such a case, for every $x \in X$, the set

$$
\left\{q \in \mathbb{R}: F_{q}^{*}(x) \leq q\right\}=\left\{q \in \mathbb{R}: \forall p \in X^{*} p . x-q \leq F(p, q)\right\}
$$

is closed, so that its infimum $r:=F^{\%}(x)$ is attained when it is finite.

Let us present characterizations of functions $F$ on $Y:=X^{*} \times \mathbb{R}$ which are equal to their flat biconjugates. The case of $F^{\text {bb }}$ is simple.

Proposition 2.11. A function $F$ on $X^{*} \times \mathbb{R}$ is equal to $F^{b b}$ iff it is separately l.s.c. on $X^{*} \times \mathbb{R}$ (with $X^{*}$ endowed with the weak* topology), is a proper (or identically $-\infty$ ) sublinear function of its first variable and is nondecreasing in its second variable. Then $F$ is jointly l.s.c. 
Proof. The necessary conditions being given in Lemma 2.1, it remains to prove the sufficient conditions. The case of $F^{b b}$ is treated in [127]. Let us give a proof for completeness. Since $F \mapsto F^{b}$ is the reverse duality associated with $f \mapsto f^{b}$, we have $F^{b b} \leq F$. Let us prove the reverse inequality by showing that, under our assumption on $F$, for every $(p, q) \in Y, t<F(p, q)$ we have $t \leq F^{b b}(p, q)$. Let $f:=F^{b}$ and for $r \in \mathbb{R}$ let

$$
S_{r}:=[f \leq r]=\partial^{c} F_{r+0}(0)=\left\{x \in X: p^{\prime} . x \leq F\left(p^{\prime}, s\right) \forall p^{\prime} \in X^{*}, \forall s>r\right\} .
$$

Since $t<F(p, q)$ and since $F(p, \cdot)$ is l.s.c. we can find $r<q$ such that $t<F(p, r)$. Since $F_{r}$ is weak* l.s.c., $^{\circ}$ proper and sublinear, one has $F_{r}(p)=\sup \left\{p . x: x \in S_{r}\right\}$ and there exists $x \in S_{r}$ such that $p . x>t$. Thus $f(x) \leq r<q$, hence $f^{b}(p, q) \geq p . x>t$ or $F^{b b}(p, q)>t$. The last assertion is contained in Lemma 2.1 (d).

Corollary 2.12. A function $F$ on $X^{*} \times \mathbb{R}$ is equal to $F^{\div} \div$iff it is separately l.s.c. on $X^{*} \times \mathbb{R}$ (with $X^{*}$ endowed with the weak* topology), and if there exists a proper (or identically $-\infty)$ l.s.c. function $G$ which is sublinear in its first variable and is nondecreasing in its second variable such that for every $(p, q) \in X^{*} \times \mathbb{R}$ one has $F(p, q)=\sup _{r<q}(G(p, r)-r)$.

Proof. If $F=F^{\div \div}$, setting $f:=F^{\div}, G:=f^{b}$, from Lemma 2.2 we see that the conditions are necessary. Conversely, if a function $G$ satisfying the conditions of the statement exists, we have $G=G^{\text {bb }}$ by the preceding proposition. Setting $f:=G^{b}$, so that $G=f^{b}$, by Lemma 2.2 we have $f^{\div}(p, q)=\sup _{r<q}(G(p, r)-r)=F(p, q)$, hence $F^{\div \div}=f \div \div \div=f \div=F$.

The case of the sharp conjugate is more delicate. We will first give criteria ensuring that a function $F$ : $X^{*} \times \mathbb{R} \rightarrow \overline{\mathbb{R}}$ satisfies $F^{\sharp \sharp} \leq F$.

Proposition 2.13. For any function $F: X^{*} \times \mathbb{R} \rightarrow \overline{\mathbb{R}}$ satisfying $F_{q+0}^{*} \geq F_{q}^{*}$ for every $q \in \mathbb{R}$ one has $F^{\sharp \sharp} \leq F$. Conversely, if for every $q \in \mathbb{R}$ the function $F_{q}:=F(\cdot, q)$ is positively homogeneous and if $F^{\sharp \sharp} \leq F$, then one has $F_{q+0}^{*} \geq F_{q}^{*}$ for every $q \in \mathbb{R}$.

Proof. Let $f:=F^{\sharp}$. Let us first suppose that for every $q \in \mathbb{R}$ the relation $F_{q+0}^{*} \geq F_{q}^{*}$ holds. Let $(p, q) \in X^{*} \times \mathbb{R}$ be such that $F^{\sharp \sharp}(p, q)>-\infty$. Then $[f \leq q]$ is nonempty and for any $x \in[f \leq q]$ we have $F_{q}^{*}(x) \leq F_{q+0}^{*}(x) \leq 0$, what implies $p . x \leq F_{q}(p)$. Hence $F^{\sharp \sharp}(p, q)=f^{\sharp}(p, q) \leq F_{q}(p)$ and this inequality also holds when $F^{\sharp \sharp}(p, q)=-\infty$. Thus $F^{\sharp \sharp} \leq F$.

Now suppose $F^{\sharp \sharp} \leq F$ and that for every $q \in \mathbb{R}$ the function $F_{q}:=F(\cdot, q)$ is positively homogeneous in the sense adopted above. Given $x \in X$ let us prove that $F_{q+0}^{*}(x) \geq F_{q}^{*}(x)$. We may suppose that $F_{q+0}^{*}(x)<+\infty$. There exists no $r>q$ such that $F_{r}$ is identically equal to $-\infty$ since otherwise $F_{q+0}$ would have the same property and we would have $F_{q+0}^{*}(x)=+\infty$. Thus $F_{r}(0)=0$ for all $r>q$ and $F_{q+0}(0)=0: F_{q+0}$ is also positively homogeneous and $F_{q+0}^{*}(x)=0$. This ensures that $x \in[f \leq q]$; thus, for every $p \in X^{*}$ we have $f^{\sharp}(p, q) \geq p$. $x$, hence $F(p, q) \geq F^{\sharp \sharp}(p, q)=f^{\sharp}(p, q) \geq p . x$. Therefore $F_{q}^{*}(x) \leq 0=F_{q+0}^{*}(x)$.

In the sequel we say that a function $F$ on a $X^{*} \times \mathbb{P}$ is right lower regular (for the sequential weak* convergence on $\left.X^{*}\right)$ if for every $(p, q) \in X^{*} \times \mathbb{P}$ we have

$$
F(p, q) \geq \liminf _{\left(p^{\prime}, q^{\prime}\right) \rightarrow\left(p, q_{+}\right)} F\left(p^{\prime}, q^{\prime}\right) .
$$

This property is satisfied whenever $F$ is u.s.c. in its second variable, but is weaker. It is satisfied if for each $p \in X^{*}$ the function $F(p, \cdot)$ is right lower regular in the sense that for each $q \in \mathbb{R}$ one has $F(p, q) \geq \liminf _{r \rightarrow q_{+}} F(p, r)$. The observation that if $k(\cdot)$ is a concave function, then it satisfies the related condition $k(q) \geq \liminf _{r \rightarrow q, r \neq q} k(r)$ on $k^{-1}(\mathbb{R})$ goes back to the work of Fenchel; see also [103]). Thus, it follows from Proposition 2.21 below that if $F=f^{\sharp}$ or $F=f^{b}$ for a convex function $f$, then $F$ is right lower regular.

Corollary 2.14. Any function $F: X^{*} \times \mathbb{R} \rightarrow \overline{\mathbb{R}}$ which is right lower regular satisfies $F_{q+0}^{*} \geq F_{q}^{*}$ for every $q \in \mathbb{R}$, hence $F^{\sharp \sharp} \leq F$. If $F: X^{*} \times \mathbb{R} \rightarrow \overline{\mathbb{R}}$ is nondecreasing in its second variable and is sublinear and weak ${ }^{*}$ l.s.c. in its first variable, then

$$
F^{\sharp \sharp}=F \Longleftrightarrow F_{q+0}^{*}=F_{q}^{*} \text { for every } q \in \mathbb{R} \text {. }
$$


Proof. Suppose $F$ is right lower regular. Let $x \in X, q, s \in \mathbb{R}$ be such that $s \geq F_{q+0}^{*}(x)$ and let us prove that $s \geq F_{q}^{*}(x)$ or, equivalently, that for every $p \in X^{*}$ one has $F(p, q) \geq p . x-s$. Since $F$ is right lower regular we can find a sequence $\left(\left(p_{n}, q_{n}\right)\right) \rightarrow(p, q)$ such that $F(p, q) \geq \liminf _{n} F\left(p_{n}, q_{n}\right)$ and $q_{n}>q$ for each $n$. Now, for every $n \in \mathbb{N}$, we have $F\left(p_{n}, q_{n}\right) \geq F\left(p_{n}, q+0\right) \geq p_{n} . x-s$. Thus, passing to the limit, $F(p, q) \geq p . x-s$, so that $s \geq F_{q}^{*}(x), F_{q+0}^{*} \geq F_{q}^{*}$ and $F^{\sharp \sharp} \leq F$ by the preceding proposition. The equivalence is then a consequence of the proposition and of the fact that $F_{q+0}^{*} \leq F_{q}^{*}$ when $F$ is nondecreasing in its second variable and of the fact that $F_{q}$ is the support function of $\left[F_{q}^{*} \leq 0\right]$ when $F_{q}$ is sublinear and weak* l.s.c.

Corollary 2.15. A function $F$ on $X^{*} \times \mathbb{R}$ is equal to $F^{\% \%}$ iff $F_{r+0}^{*}=F_{r}^{*}$ for every $r \in \mathbb{R}$ and if there exists a proper (or identically $-\infty$ ) function $G$ which is weak* l.s.c., sublinear in its first variable, is nondecreasing in its second variable and such that for every $(p, q) \in X^{*} \times \mathbb{R}$ one has $G_{q+0}^{*}=G_{q}^{*}$ and $F(p, q)=\sup _{r \leq q}(G(p, r)-r)$.

Proof. Again, the necessary condition stems from Lemma 2.2. Let us show it is sufficient. Setting $f:=G^{\sharp}$, the preceding corollary ensures that $G=f^{\sharp}$. Then, for every $(p, q) \in X^{*} \times \mathbb{R}$ one has $F(p, q)=$ $\sup _{r \leq q}(G(p, r)-r)=\sup _{r \leq q}\left(f^{\sharp}(p, r)-r\right)=f^{\%}(p, q)$. Moreover, $F^{\%}=f^{\% \%} \leq f$, hence $F^{\% \%} \geq f^{\%}=F$.

Let us observe that the notion of right lower regularity is a weakening of the notion of right upper epicontinuity which means that for every $q \in \mathbb{R}$ one has $F_{q} \geq e-\lim \sup _{r \rightarrow q_{+}} F_{r}$ or (see section 8 below)

$$
\text { epi } F_{q} \subset \liminf _{r \rightarrow q_{+}} \text {epi } F_{r} \text {, }
$$

i.e. for any $(p, q) \in X^{*} \times \mathbb{R}$, any $s \geq F(p, q)$ and any sequence $\left(q_{n}\right) \rightarrow q_{+}$there exists a sequence $\left(p_{n}, s_{n}\right) \rightarrow(p, s)$ such that $s_{n} \geq F\left(p_{n}, q_{n}\right)$ for each $n \in \mathbb{N}$. In fact, right lower regularity means that $F_{q} \geq e_{w}-\liminf _{r \rightarrow q_{+}} F_{r}$ or

$$
\text { epi } F_{q} \subset \limsup _{r \rightarrow q_{+}} \text {epi } F_{r} \text {. }
$$

For a function $F: X^{*} \times \mathbb{R} \rightarrow \overline{\mathbb{R}}$ which is nondecreasing in its second variable, the two notions are equivalent. If moreover $F$ is jointly weak* l.s.c. in its two variables, right upper epicontinuity coincides with right epicontinuity (i.e. epi $F_{q}=\lim _{r \rightarrow q_{+}}$epi $F_{r}$ for every $q \in \mathbb{R}$ ).

We can deduce from what precedes a characterization of the equality $F^{\sharp \sharp}=F$.

Proposition 2.16. ( [95], [98]) Suppose $X$ is reflexive. Then, for a function $F: X^{*} \times \mathbb{R} \rightarrow \overline{\mathbb{R}}$, the following assertions are equivalent:

(a) $F^{\sharp \sharp}=F$;

(b) there exists a l.s.c. quasiconvex function $f$ on $X$ such that $f^{\sharp}=F$;

(c) there exists a function $f$ on $X$ such that $f^{\sharp}=F$;

(d) $F$ is right epi-continuous, weak* l.s.c. and sublinear in its first variable and nondecreasing in its second variable;

(e) $F$ is right lower regular, weak* l.s.c. and sublinear in its first variable and nondecreasing in its second variable.

Proof. The implications (a) $\Rightarrow(\mathrm{b}) \Rightarrow(\mathrm{c}),(\mathrm{d}) \Rightarrow(\mathrm{e})$ are obvious. (e) $\Rightarrow(\mathrm{d})$ and $(\mathrm{e}) \Rightarrow(\mathrm{a})$ have just been observed. (c) $\Rightarrow$ (b) is obtained by noting that for the l.s.c. quasiconvex hull $h$ of $f$ one has $h^{\sharp}=f^{\sharp}$. (b) $\Rightarrow$ (d) is a consequence of the continuity of the Fenchel conjugate and of the fact that for every $r \in \mathbb{R}$ one has $[f \leq r]=\lim _{s \backslash r}[f \leq s]$, hence $\iota_{[f \leq r]}=$ epi $-\lim _{s \backslash r} \iota_{[f \leq s]}$.

We deduce from Corollary 2.14 the following consequence.

Corollary 2.17. If $f$ is a l.s.c. quasiconvex function $f$ on $X$ and if $F=f^{\sharp}$, then for every $q \in \mathbb{R}$ one has $F_{q+0}^{*}=F_{q}^{*}$. In other words, for any $x \in X$, the function $q \mapsto F_{q}^{*}(x)$ is l.s.c.

Calculus rules for the dualities we described may be useful when dealing with the explicit formulas we study. The rules with the classical Legendre-Fenchel transform are well known. The ones concerning the quasiconvex dualities are less familiar ( [94], [107]). Let us present a sample. 
Lemma 2.18. (a) Let $\left(f_{i}\right)_{i \in I}$ be a family of functions on $X$ and let $f:=\inf _{i \in I} f_{i}$. Then $f^{b}=\sup _{i \in I} f_{i}^{b}$. If for each $x \in X$ the infimum $\inf _{i \in I} f(x)$ is attained, then $f^{\sharp}=\sup _{i \in I} f_{i}^{\sharp}$.

(b) Let $\left(F_{i}\right)_{i \in I}$ be a family of functions on $Y$ and let $F:=\inf _{i \in I} F_{i}$. Then $F^{b}=\sup _{i \in I} F_{i}^{b}=\sup _{i \in I} F_{i}^{\sharp}=F^{\sharp}$.

(c) In particular, for any $f \in \overline{\mathbb{R}}^{X}, F \in \overline{\mathbb{R}}^{Y}, c \in \mathbb{R}$ one has $(f \wedge c)^{\sharp}=f^{\sharp} \vee c^{\sharp}$ with $c^{\sharp}(p, q)=-\infty$ for $q<c$, $c^{\sharp}(p, q)=+\infty$ for $p \neq 0, q \geq c, c^{\sharp}(0, q)=0$ for $q \geq c$ and $(f \wedge c)^{b}=f^{b} \vee c^{b}$ with $c^{b}(p, q)=-\infty$ for $q \leq c$, $c^{b}(p, q)=+\infty$ for $p \neq 0, q \geq c, c^{b}(0, q)=0$ for $q \geq c$ while $(F \wedge c)^{b}=(F \wedge c)^{\sharp}=+\infty$ for $c<0,(F \wedge c)^{\sharp}=F^{\sharp}$ for $c>0$.

Proof. Assertion b) and the first part of assertion a) have already been noted. The second part of a) follows from the fact that, under its assumption, for any $(p, q) \in Y$ one has $[f \leq q]=\bigcup_{i \in I}\left[f_{i} \leq q\right]$.

In the following rules for composition we use the lower and upper quasi-inverses $\varphi^{e}, \varphi^{h}$ of a nondecreasing function $\varphi: \overline{\mathbb{R}} \rightarrow \overline{\mathbb{R}}$; they are given by

$$
\begin{aligned}
\varphi^{e}(s) & :=\sup \{r \in \overline{\mathbb{R}}: \varphi(r)<s\} \quad \text { for } s \in \overline{\mathbb{R}} \\
\varphi^{h}(s) & :=\inf \{t \in \overline{\mathbb{R}}: \varphi(t)>s\} \quad \text { for } s \in \overline{\mathbb{R}}
\end{aligned}
$$

with the usual convention $\inf \varnothing=+\infty, \sup \varnothing=-\infty$. If $\varphi$ is defined on $\mathbb{R}$ the similar definitions in which $r$ and $t$ are taken in $\mathbb{R}$ give the same quasi-inverses as in extending $\varphi$ by $\varphi(-\infty)=-\infty, \varphi(+\infty)=+\infty$. One easily sees that $\varphi^{e} \leq \varphi^{h}$. Moreover, since for any $s \in \overline{\mathbb{R}}$ one has $] \varphi^{e}(s), \varphi^{h}(s)\left[\subset \varphi^{-1}(s)\right.$, one gets that when $\varphi: \overline{\mathbb{R}} \rightarrow \mathbb{R}$ is (strictly) increasing then $\varphi^{e}$ and $\varphi^{h}$ coincide and coincide on $\varphi(\overline{\mathbb{R}})$ with $\varphi^{-1}$ considered as a function.

Lemma 2.19. ([106] Prop. 5.3) Let $f=\varphi \circ g$ where $\varphi: \overline{\mathbb{R}} \rightarrow \overline{\mathbb{R}}$ is nondecreasing and $g: X \rightarrow \overline{\mathbb{R}}$ is convex proper. If $\left.\varphi^{e}(r) \in\right] \inf g, \infty[$ then

$$
f^{b}\left(x^{*}, r\right)=g^{b}\left(x^{*}, \varphi^{e}(r)\right)=g^{\sharp}\left(x^{*}, \varphi^{e}(r)\right),
$$

and if $\left.\varphi^{h}(r) \in\right] \inf g, \infty[$ one has

$$
f^{\sharp}\left(x^{*}, r\right)=g^{\sharp}\left(x^{*}, \varphi^{h}(r)\right)=g^{b}\left(x^{*}, \varphi^{h}(r)\right) .
$$

Lemma 2.20. Let $F: X \times \mathbb{R} \rightarrow \overline{\mathbb{R}}$ be nondecreasing in its second variable and let $G: Y \rightarrow \overline{\mathbb{R}}$ be given by $G(p, q)=F(p, \psi(q))$ where $\psi: \mathbb{R} \rightarrow \mathbb{R}$ is nondecreasing. Then one has $G^{\sharp} \leq \psi^{h} \circ F^{\sharp}$. When $\psi$ is increasing one has $G^{b}=G^{\sharp}=\psi^{e} \circ F^{\sharp}=\psi^{h} \circ F^{\sharp}$.

Proof. Let $x \in X$ and let $s:=F^{\sharp}(x)$. When $s=+\infty$ we have $\psi(s)=+\infty$ and the inequality is valid, so that we may suppose $s<+\infty$. For every $t \in \mathbb{R}$ such that $\psi(t)>s$ we have $G_{t}^{*}(x)=F_{\psi(t)}^{*}(x) \leq 0$, hence $G^{\sharp}(x) \leq t$. Taking the infimum over $t \in \psi^{-1}(] s,+\infty[)$, we get $G^{\sharp}(x) \leq \psi^{h}(s)$. On the other hand, when $r \in \mathbb{R}$ satisfies $\psi(r)<s$, we have $G_{t}^{*}(x)=F_{\psi(t)}^{*}(x)>0$, hence $G^{\sharp}(x) \geq t$. Taking the supremum over $r \in \psi^{-1}(]-\infty, s[)$, we get $G^{\sharp}(x) \geq \psi^{e}(s)$. When $\psi$ is increasing one has $\psi^{e}=\psi^{h}$ since otherwise one could find $q<q^{\prime}$ and $s \in \overline{\mathbb{R}}$ such that $\psi^{e}(s)<q<q^{\prime}<\psi^{h}(s)$.

Let us give a means to detect transforms of convex functions among transforms of l.s.c. quasiconvex functions.

Proposition 2.21. (a) If $f: X \rightarrow \overline{\mathbb{R}}$ is convex, then $f^{b}, f^{\sharp}$ and $f^{\div}, f^{\%}$ are concave and nondecreasing in their second variable.

(b) If $F: X^{*} \times \mathbb{R} \rightarrow \overline{\mathbb{R}}$ is concave and nondecreasing in its second variable, then $f:=F^{b}=F^{\sharp}$ and $g:=F^{\div}=F^{\%}$ are convex.

(c) A l.s.c. quasiconvex function $f$ is convex if, and only if, $f^{b}$ (resp. $f^{\sharp}, f^{\div}, f^{\%}$ ) are concave in their second variable. 
Proof. (a) Given $p \in X^{*}, q_{0}, q_{1} \in \mathbb{R}$ and $t \in[0,1]$, for every $x_{0} \in\left[f \leq q_{0}\right], x_{1} \in\left[f \leq q_{1}\right]$ one has $x_{t}:=(1-t) x_{0}+t x_{1} \in\left[f \leq(1-t) q_{0}+t q_{1}\right]$, hence

$$
\begin{aligned}
f^{\sharp}\left(p,(1-t) q_{0}+t q_{1}\right) & \geq p \cdot x_{t}=(1-t) p \cdot x_{0}+t p \cdot x_{1}, \\
f^{\%}\left(p,(1-t) q_{0}+t q_{1}\right) & \geq p \cdot x_{t}-f\left(x_{t}\right) \geq(1-t)\left(p \cdot x_{0}-f\left(x_{0}\right)\right)+t\left(p \cdot x_{1}-f\left(x_{1}\right)\right) .
\end{aligned}
$$

Taking the supremum over $x_{0}$ and $x_{1}$, we get $f^{\sharp}\left(p,(1-t) q_{0}+t q_{1}\right) \geq(1-t) f^{\sharp}\left(p, q_{0}\right)+t f^{\sharp}\left(p, q_{1}\right)$ and $f^{\%}(p,(1-$ t) $\left.q_{0}+t q_{1}\right) \geq(1-t) f^{\%}\left(p, q_{0}\right)+t f^{\%}\left(p, q_{1}\right)$. The proofs for $f^{b}$ and $f \div$ are similar.

(b) Let $x_{0}, x_{1} \in X$ and $t \in[0,1]$ and let $x_{t}:=(1-t) x_{0}+t x_{1}$. For any $r_{i}>f\left(x_{i}\right)$ one has $F_{r_{i}}^{*}\left(x_{i}\right) \leq 0$ for $i=0,1$. Thus, for every $p \in X^{*}$ one has $p . x_{i} \leq F\left(p, r_{i}\right)$, hence, by concavity, $p . x_{t} \leq F\left(p,(1-t) r_{0}+t r_{1}\right)$ and $F_{q}^{*}\left(x_{t}\right) \leq 0$ for $q \geq(1-t) r_{0}+t r_{1}$. This shows that $f\left(x_{t}\right) \leq(1-t) r_{0}+t r_{1}$. Thus $f$ is convex.

Now let $r_{i}>g\left(x_{i}\right)(i=0,1)$ and let $r_{t}:=(1-t) r_{0}+t r_{1}$. Since $F_{r_{i}}^{*}\left(x_{i}\right) \leq r_{i}$, for every $p \in X^{*}$ we have $p . x_{i}-r_{i} \leq F\left(p, r_{i}\right)$. By concavity of $F(p, \cdot)$ we get $p x_{t}-r_{t} \leq F\left(p, r_{t}\right)$. Thus $F_{r_{t}}^{*}\left(x_{t}\right) \leq r_{t}$ and $F^{\%}\left(x_{t}\right) \leq r_{t}$. Thus $g:=F^{\%}$ is convex.

(c) The assertion follows from (a), (b) and the fact that $f=f^{b b}=f^{\sharp \sharp}$.

The dualities described above give rise to various notions of subdifferentials, following a general process devised by Martínez-Legaz and Singer in a series of papers ( [81]- [84]). This process is extended to quasidualities in [94]. We refer to these papers for this general construction and for the links with subdifferentials which are classical in generalized convexity theory.

\section{Some Notions of SOlution}

It is crucial to define a concept of generalized solution of equation (1) and a concept of solution for the system (1)-(2). Several concepts have been introduced. Obviously, the best uniqueness results are obtained by using a notion as loose as possible while the best existence results are those which adopt a definition as stringent as possible.

The usual concepts of solution are the notions of generalized solution ( [60], [13], [51]...) and of viscosity solution ( [40], [39], [37], [16], [13], [51]...); the notion of bilateral solution (or lsc solution) due to Barron and Jensens ( [19]) is also important. Generalized solutions essentially use locally Lipschitzian functions, so that the Rademacher theorem ensures the existence of the derivative almost everywhere when $X$ is finite dimensional; when $X$ is a separable Banach space, the use of Haar null sets or of Gaussian null sets would probably provide extensions. However, the central notion is the notion of viscosity solution; it uses a comparison function $\varphi$, and for this reason it is in the spirit of what is done with test functions for the study of partial differential equations. We will rather use the closely related notion of subdifferential which is central in nonsmooth analysis. Another approach consists in taking generalized directional derivatives, for instance of Hadamard (or contingent or Dini) type (see [70], [55]- [54], [78]). This last approach cannot be related to methods using subdifferentials such as the ones in generalized convexity theory, or the Fréchet subdifferential, the limiting Fréchet subdifferential or the Ioffe subdifferential. For this reason, we use an unspecified subdifferential $\partial^{\text {? }}$ considered as a mapping $\partial^{?}: \mathcal{F}(X) \times X \rightarrow 2^{X^{*}}$ where $\mathcal{F}(X)$ is some function space on $X$. Let us present the subdifferentials we will use.

Recall that the lower (or contingent) Hadamard derivative of a function $f: Z \rightarrow \overline{\mathbb{R}}$ on a normed vector space $Z$ at some $z \in f^{-1}(\mathbb{R})$ is given by

$$
f^{\prime}(z, w):=\liminf _{(t, v) \rightarrow\left(0_{+}, w\right)} \frac{1}{t}[f(z+t v)-f(z)]
$$

and that the Hadamard (or contingent) subdifferential of $f$ at $z$ is given by

$$
\partial f(z):=\left\{z^{*} \in Z^{*}: z^{*} \leq f^{\prime}(z, \cdot)\right\} .
$$


This set contains the Fréchet subdifferential of $f$ at $z$ which is the set given by

$$
\partial^{-} f(z):=\left\{z^{*} \in Z^{*}: \liminf _{\|u\| \rightarrow 0_{+}} \frac{1}{\|u\|}\left[f(z+u)-f(z)-\left\langle z^{*}, u\right\rangle\right] \geq 0\right\} .
$$

Note that $\partial^{-} f(z)$ may be empty while $\partial f(z)$ is nonempty. For any normed vector space, $\partial^{-} f(z)$ contains the viscosity subdifferential of $f$ at $z$ which is the set of derivatives at $z$ of smooth functions $\varphi$ such that $f-\varphi$ attains a local minimum at $z$; in spaces with a smooth bump function, in particular in finite dimensional spaces, $\partial^{-} f(z)$ coincides with the viscosity subdifferential of $f$ at $z$.

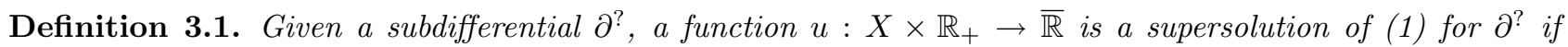
for any $(x, t) \in X \times \mathbb{P}$ and any $\left(x^{*}, t^{*}\right) \in \partial^{?} u(x, t)$ one has $t^{*}+H\left(x^{*}, u(x, t)\right) \geq 0$. Similarly, $u$ is called a subsolution for $\partial^{?}$ if for $(x, t) \in X \times \mathbb{P}$ and any $\left(x^{*}, t^{*}\right) \in-\partial^{?}(-u)(x, t)$ one has $t^{*}+H\left(x^{*}, u(x, t)\right) \leq 0$. It is a Crandall-Lions solution if it is both a supersolution and a subsolution (for $\left.\partial^{\text {? }}\right)$.

The following observation is an immediate consequence of the definition.

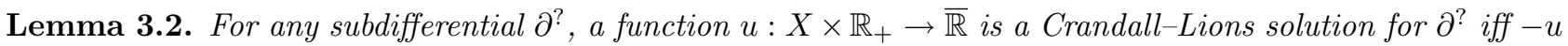
is a Crandall-Lions solution for the Hamiltonian $\widetilde{H}$ given by $\widetilde{H}(p, q)=-H(-p,-q)$.

Of course, in such a case, one takes $-g$ as initial data.

When $\partial^{\text {? }}$ is the Hadamard (or contingent) subdifferential $\partial$ we say that $u$ is a Hadamard solution or a contingent solution. The preceding observations show that any Hadamard solution is a viscosity solution; the converse is true in smooth spaces. Clearly, if $u$ is a Hadamard solution, then, for each $(x, t)$ at which $u$ is Hadamard differentiable one has

$$
\frac{\partial u}{\partial t}(x, t)+H\left(D u_{t}(x), u(x, t)\right)=0 .
$$

Although the preceding notions seem to be the most important concepts, it may be useful to consider other ones.

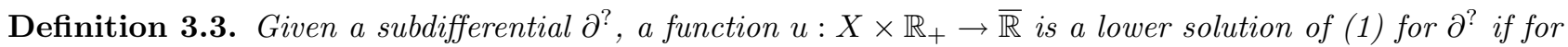
any $(x, t) \in X \times \mathbb{P}$ and any $\left(x^{*}, t^{*}\right) \in \partial^{?} u(x, t)$ one has $t^{*}+H\left(x^{*}, u(x, t)\right) \leq 0$. It is an upper solution of $(1)$ for $\partial^{?}$ if for any $(x, t) \in X \times \mathbb{P}$ and any $\left(x^{*}, t^{*}\right) \in-\partial^{?}(-u)(x, t)$ one has $t^{*}+H\left(x^{*}, u(x, t)\right) \geq 0$.

Any convex upper solution of (1) for a subdifferential $\partial^{\text {? }}$ larger than the Hadamard subdifferential is easily seen to be a supersolution for $\partial^{\text {? }}$. Such a fact explains why upper solutions are not considered in the literature. However, any convex subsolution for $\partial^{\text {? }}$ is also a lower solution for $\partial^{?}$ and subsolutions have been given more attention than lower solutions.

Note that if $u$ is a lower solution and a supersolution for $\partial^{\text {? }}$, then for any $(x, t) \in X \times \mathbb{P}$ and any $\left(x^{*}, t^{*}\right) \in$ $\partial^{?} u(x, t)$ one has $t^{*}+H\left(x^{*}, u(x, t)\right)=0$. Then one says that $u$ is a bilateral solution or a subdifferential solution for $\partial^{\text {? }}$. This notion may depend on the choice of the subdifferential. For instance, if $f: X \rightarrow \mathbb{R}$ is nowhere Hadamard subdifferentiable but at 0 and if $f$ is Hadamard differentiable at 0 but not Fréchet subdifferentiable there, the function $u$ given by $u(x, t):=f(x)-t c$ with $c \in \mathbb{R}, c \neq H(D f(0))$ is a Fréchet subdifferential solution of (1)-(2) for $g:=f$, but it is not a Hadamard subdifferential solution. It may also happen that a Crandall-Lions solution for the Hadamard subdifferential is not a lower solution.

Example 3. Let $g$ and $H$ be given by $g(x)=\|x\|, H(p)=-\|p\|$. Then $v$ given by $v(x, t):=\|x\|+t$ is a Crandall-Lions solution for the Hadamard subdifferential and $v(\cdot, 0)=g$; moreover, for each $t>0$ and each $x$ such that $\|\cdot\|$ is differentiable at $x$ one has $\frac{\partial}{\partial t} v(x, t)+H\left(D v_{t}(x)\right)=0$; however $v$ is not a Hadamard lower solution since $(p, 1) \in \partial v(0, t)$ for each $p$ in the unit ball of $X^{*}$ and for each $t \geq 0$. However, for each $(x, t) \in X \times \mathbb{P}$ one has

$$
\inf \{q+H(p):(p, q) \in \partial v(x, t)\}=0 .
$$

We observe that $f$ is lower semicontinuous (l.s.c.) at $z$ whenever $\partial^{-} f(z)$ is nonempty; the nonemptiness of the Hadamard subdifferential $\partial f(z)$ entails just a directional lower semicontinuity. Nevertheless, a lack of 
semicontinuity of a potential solution $u$ at some point $(x, t)$ is not an obstacle: it just means that the condition of the preceding definition is automatically satisfied at $(x, t)$.

Example 4. Let $X=\mathbb{R}$ and let $g$ and $H$ be given by $H(p)=|p|, g(x)=\chi_{\mathbb{R}_{+}}(x)$ where $\chi_{\mathbb{R}_{+}}$is the characteristic function of the set $\mathbb{R}_{+}$of nonnegative real numbers: $\chi_{\mathbb{R}_{+}}(x)=0$ for $x<0, \chi_{\mathbb{P}}(x)=1$ for $x \geq 0$. Then $u$ given by $u(x, t)=\chi_{T}(x)$ where $T$ is the interval $[t,+\infty[$ of $\mathbb{R}$ is a bilateral solution to (1) for the Hadamard subdifferential which is not l.s.c..

The following theorem is close in spirit to results of Barron and Jensen ( [19], [18, Thm 16]) and Frankowska [55] (see also [54], [56], [57]). In that statement, a Banach space is said to be Fréchet smooth if it carries a non null Fréchet differentiable function which is Lipschitzian and has a bounded support.

Theorem 3.4. Suppose $H$ is l.s.c., convex, and $X$ is a Fréchet smooth reflexive Banach space. Then, any l.s.c. Fréchet lower solution u to (1) is a Fréchet subsolution to (1). Conversely any u.s.c. Fréchet subsolution to (1) is a Fréchet lower solution u to (1).

When $H$ is concave and u.s.c., an analogous result holds: any u.s.c. Fréchet upper solution is a Fréchet supersolution and conversely, any l.s.c. Fréchet supersolution is a Fréchet upper solution.

Proof. Let $(x, t) \in X \times \mathbb{P}$ and let $(p, q) \in-\partial^{-}(-u)(x, t)$. Theorem 3.6.5 of [28] asserts that for every $\varepsilon>0$ one can find some $\left(p^{\prime}, q^{\prime}\right)$ in the convex hull of $\partial^{-} u(B((x, t), \varepsilon))$ such that $\left\|\left(p^{\prime}, q^{\prime}\right)-(p, q)\right\| \leq \varepsilon$. Thus, there exist a finite family $\left(\left(x_{i}, t_{i}\right)\right)_{i \in I}$ in $B((x, t), \varepsilon)$, corresponding $\left(p_{i}, q_{i}\right) \in \partial^{-} u\left(x_{i}, t_{i}\right)$ and a family $\left(\lambda_{i}\right)_{i \in I}$ of $[0,1]$ with sum 1 such that

$$
\left(p^{\prime}, q^{\prime}\right)=\sum_{i \in I} \lambda_{i}\left(p_{i}, q_{i}\right)
$$

Since $u$ is a Fréchet lower solution of (1), one has $q_{i}+H\left(p_{i}\right) \leq 0$ for each $i \in I$. Then, by convexity, $q^{\prime}+H\left(p^{\prime}\right) \leq 0$. Since $\varepsilon>0$ is arbitrary, by lower semicontinuity of $H$ we deduce that $q+H(p) \leq 0$. The converse is similar. Given $(p, q) \in \partial^{-} u(x, t)$, setting $v:=-u$ we have $(-p,-q) \in-\partial^{-}(-v)(x, t)$. Then we can find $\left(p^{\prime}, q^{\prime}\right) \in B((-p,-q), \varepsilon)$ and $\left(\lambda_{i}\right)_{i \in I},\left(p_{i}, q_{i}\right) \in \partial^{-} v\left(x_{i}, t_{i}\right)$ such that (13) holds. Then $-q_{i}+H\left(-p_{i}\right) \leq 0$. By convexity and lower semicontinuity of $H$ we obtain $-q^{\prime}+H\left(-p^{\prime}\right) \leq 0$ and then $q+H(p) \leq 0$.

One can also define a concept of solution using other subdifferentials, for instance, the Fenchel-Moreau subdifferential of convex analysis given by

$$
\partial^{c} f(z):=\left\{z^{*} \in Z^{*}: \forall w \in Z\left\langle z^{*}, w-z\right\rangle \leq f(w)-f(z)\right\}
$$

or the lower subdifferential of Plastria given by

$$
\partial^{<} f(z):=\left\{z^{*} \in Z^{*}: \forall w \in f^{-1}(f(z)-\mathbb{P})\left\langle z^{*}, w-z\right\rangle \leq f(w)-f(z)\right\},
$$

or the infra-differential of Gutiérrez [59] given by

$$
\partial^{\leq} f(z):=\left\{z^{*} \in Z^{*}: \forall w \in f^{-1}\left(f(z)-\mathbb{R}_{+}\right)\left\langle z^{*}, w-z\right\rangle \leq f(w)-f(z)\right\} .
$$

We will also use the following variants of the Greensberg-Pierskalla subdifferential

$$
\partial^{\circledast} f(z)=N^{r}([f<f(z)], z), \quad \partial^{\nu} f(z)=N^{r}([f \leq f(z)], z),
$$

where $N^{r}(S, z)$ is the polar cone $\left(T^{r}(S, z)\right)^{0}$ of the radial tangent cone $T^{r}(S, z)$ to $S$ at $z$ given by

$$
T^{r}(S, z):=\{w \in X: \exists \varepsilon \in \mathbb{P} \quad \forall t \in] 0, \varepsilon[\quad z+t w \in S\} .
$$

Note that since $T^{r}(S, z)$ is contained in the tangent cone $T(S, z):=\lim \sup _{t \rightarrow 0_{+}} t^{-1}(S-z)$, the radial normal cone $N^{r}(S, z)$ is larger than the usual normal cone $N(S, z):=(T(S, z))^{0}$. When $S$ is convex, both cones coincide 
with the polar cone to the tangent cone and $T(S, z)=\operatorname{cl} T^{r}(S, z)=\operatorname{cl}\left(\mathbb{R}_{+}(S-z)\right)$. Therefore, for a quasiconvex function $f, \partial^{\circledast} f(z)$ and $\partial^{\nu} f(z)$ coincide with the subdifferentials studied in [93], [94]:

$$
\begin{aligned}
& \partial^{\circledast} f(z)=\left\{z^{*} \in Z^{*}: \forall w \in[f<f(z)]\left\langle z^{*}, w-z\right\rangle \leq 0\right\}, \\
& \partial^{\nu} f(z)=\left\{z^{*} \in Z^{*}: \forall w \in[f \leq f(z)]\left\langle z^{*}, w-z\right\rangle \leq 0\right\} .
\end{aligned}
$$

Moreover

$$
\mathbb{R}_{+} \partial^{<} f(z) \subset \partial^{\circledast} f(z), \quad \mathbb{R}_{+} \partial^{\leq} f(z) \subset \partial^{\nu} f(z) \subset \partial^{\circledast} f(z) .
$$

When $f$ is convex, $f$ finite at $z$, most subdifferentials, in particular $\partial f(z)$ and $\partial^{-} f(z)$, coincide with $\partial^{c} f(z)$. That is not the case for $\partial^{<} f(z)$ and $\partial^{\leq} f(z)$ : if $z$ is not a minimizer of $f$, then $\partial^{<} f(z)=\partial^{\leq} f(z)=\left[1,+\infty\left[\partial^{c} f(z)\right.\right.$. Let us observe that for any function $f$ finite at $z$ one also has

$$
\mathbb{R}_{+} \partial f(z) \subset \partial^{\nu} f(z)
$$

in fact, for any $z^{*} \in \partial f(z)$ and any $v \in T^{r}(S, z)$ with $S:=[f \leq f(z)]$, or even for any $v$ in the tangent cone $T(S, z)$ to $S$ at $z$ there exist sequences $\left(r_{n}\right) \rightarrow 0_{+},\left(v_{n}\right) \rightarrow v$ such that $z+r_{n} v_{n} \in S$ for each $n$, so that $\left\langle z^{*}, v\right\rangle \leq f^{\prime}(z, v) \leq 0$. It ensues that the following concept of normal supersolution is stronger than the concept of Hadamard supersolution (and a fortiori stronger than the notion of viscosity supersolution).

Definition 3.5. A function $u$ on $X \times \mathbb{R}$ is a normal supersolution (resp. a normal lower solution) for (1) if for any $(x, t) \in X \times \mathbb{P}$ and any $\left(x^{*}, t^{*}\right) \in \partial^{\nu} u(x, t):=N^{r}([u \leq u(x, t)],(x, t))$ one has $t^{*}+H\left(x^{*}, u(x, t)\right) \geq 0$ $\left(\right.$ resp. $\left.t^{*}+H\left(x^{*}, u(x, t)\right) \leq 0\right)$.

Since our approach relies on a notion of subdifferential, we can invoke the known rules for the calculus of subdifferentials in order to get properties of solutions. The following results are simple instances of such a means; we refer to [94] and [107] for the convex case.

Proposition 3.6. Let $\left(u_{i}\right)_{i \in I}$ be a family of supersolutions (resp. lower solutions) for the Hadamard subdifferential $\partial$ of (1) and let $u=\inf _{i} u_{i}$ be an exact infimum, i.e. be such that for every $(x, t) \in$ dom $u$ there exists $j \in I$ for which $u_{j}(x, t)=u(x, t)$. Then $u$ is a supersolution (resp. is a lower solution) for the Hadamard subdifferential. If each $u_{i}$ is a subsolution for $\partial$, then $v=\sup _{i} u_{i}$ is a subsolution for $\partial$ if the supremum is exact (i.e. attained at each point of dom u).

Similar results hold for any other subdifferential $\partial^{\text {? }}$ which is homotone in the sense of [96]: given a n.v.s. $Z$, $z \in Z$ and two functions $u \leq v$ on $Z$ with $u(z)=v(z)$, one has $\partial^{?} u(z) \subset \partial^{?} v(z)$. In fact, given $(x, t) \in X \times \mathbb{P}$ and $(p, q) \in \partial^{?} u(x, t)$, for any $j \in I$ for which $u_{j}(x, t)=u(x, t)$ we have $(p, q) \in \partial^{?} u_{j}(x, t)$, hence $q+H(p) \geq 0$ (resp. $q+H(p) \leq 0)$ if $u_{j}$ is a supersolution (resp. a lower solution). The proof for a subsolution is similar since $-\partial^{?}(-v)(x, t) \subset-\partial^{?}\left(-u_{j}\right)(x, t)$ when $u_{j}(x, t)=v(x, t):=\sup _{i \in I} u_{i}(x, t)$. Under the (stringent) assumptions of [13] Proposition 2.13 p. 44 one can conclude that $u=\inf _{i} u_{i}$ is a subsolution when each $u_{i}$ is a subsolution and $H$ is convex and weak* lower semicontinuous. These assumptions can be relaxed when $u_{i}$ is convex for each $i \in I$; see the works of Laurent [73], Correa-Seeger, Valadier, Lions-Souganidis [77], Jofre...

Stability results can be deduced from [47], [90], [130]; see also [30], [111].

Let us rather present two results connected with composition rules. Here we assume the subdifferential satisfies natural chain rules for the precomposition with a continuously differentiable and for the postcomposition with a differentiable increasing fonction from $\mathbb{R}$ to $\mathbb{R}$. Such rules is obviously satisfied with the Fréchet and the Hadamard subdifferentials, for instance.

Lemma 3.7. Let $\varphi: \mathbb{R} \rightarrow \mathbb{R}$ be a differentiable increasing function with a differentiable inverse $\psi$. Let $\widehat{u}: X \times \mathbb{R} \rightarrow \mathbb{R}$ be a supersolution (resp. lower solution) to the equation $\frac{\partial}{\partial t} \widehat{u}(x, t)+\widehat{H}(x, D \widehat{u}(x, t), \widehat{u}(x, t))=0$. Then $u:=\varphi \circ \widehat{u}$ is a supersolution (resp. lower solution) of the equation $\frac{\partial}{\partial t} u(x, t)+H(x, D u(x, t), u(x, t))=0$, where $H(x, p, r)=\varphi^{\prime}(\psi(r)) \widehat{H}\left(x, \psi^{\prime}(r) p, \psi(r)\right)$. 
Note in particular, that $H=\widehat{H}$ when $\widehat{H}$ is positively homogeneous in its second variable.

A similar result holds for lower solutions and subsolutions. Moreover, when $\widehat{H}$ is positively homogeneous in its second variable, one has $H(x, p, r)=\widehat{H}(x, p, \psi(r))$.

Proof. The result follows from the chain rule

$$
\partial^{?} u(x, t)=\varphi^{\prime}(u(x, t)) \partial^{?} \widehat{u}(x, t) .
$$

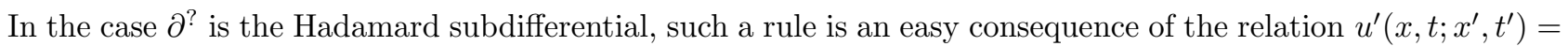
$\varphi^{\prime}(u(x, t)) \widehat{u}^{\prime}\left(x, t ; x^{\prime}, t^{\prime}\right)$.

Lemma 3.8. Let $W, \widehat{W}$ be open subsets of Banach spaces $X, \widehat{X}$ respectively and let $f: W \rightarrow \widehat{W}$ be a continuously differentiable function such that $f(W)=\widehat{W}$. Let $H: W \times X^{*} \times \mathbb{R} \rightarrow \mathbb{R}$ and $\widehat{H}: \widehat{W} \times \widehat{X}^{*} \times \mathbb{R} \rightarrow \mathbb{R}$ be such that

$$
H\left(x, \widehat{p} \circ f^{\prime}(x), r\right)=\widehat{H}(f(x), \widehat{p}, r) \quad \forall x \in W, \widehat{p} \in X^{*}, r \in \mathbb{R} .
$$

If $u:=\widehat{u} \circ f$ is a supersolution (resp. lower solution) to $\frac{\partial}{\partial t} u(x, t)+H(x, D u(x, t), u(x, t))=0$ then $\widehat{u}$ is a supersolution (resp. lower solution) to $\frac{\partial}{\partial t} \widehat{u}(x, t)+\widehat{H}(x, D \widehat{u}(x, t), \widehat{u}(x, t))=0$. The converse holds when, for every $w \in W, f^{\prime}(w)$ is surjective

PROof. Under our assumptions the contingent derivatives of $u$ and $\widehat{u}$ are related by

$$
u^{\prime}\left(z, z^{\prime}\right)=\widehat{u}^{\prime}\left(\bar{f}(z), \bar{f}^{\prime}(z) z^{\prime}\right) \quad \forall z \in W \times \mathbb{P}, z^{\prime} \in Z:=X \times \mathbb{R}
$$

where $\bar{f}(x, t)=(f(x), t)$ for $z:=(x, t) \in W \times \mathbb{P}$. It follows that $\partial \widehat{u}(\bar{f}(z)) \circ \bar{f}^{\prime}(z) \subset \partial u(z)$. Conversely, if $z^{*} \in \partial u(z)$, for each $z^{\prime} \in \operatorname{ker} \bar{f}^{\prime}(z)$ we have $z^{*} \cdot z^{\prime} \leq u^{\prime}\left(z, z^{\prime}\right)=\widehat{u}^{\prime}\left(\bar{f}(z), \bar{f}^{\prime}(z) z^{\prime}\right) \leq 0$. Since $\bar{f}^{\prime}(z)$ is surjective, there exists some $\widehat{z}^{*} \in \widehat{X}^{*} \times \mathbb{R}$ such that $z^{*}=\widehat{z}^{*} \circ \bar{f}^{\prime}(z)$ and we observe that $\widehat{z}^{*} \in \partial \widehat{u}(\bar{f}(z))$. Thus $\partial \widehat{u}(\bar{f}(z)) \circ \bar{f}^{\prime}(z)=$ $\partial u(z)$ or $(p, q) \in \partial u(x, t)$ iff there exists $(\widehat{p}, \widehat{q}) \in \partial \widehat{u}(f(x), t)$ such that $p=\widehat{p} \circ f^{\prime}(x), q=\widehat{q}$. The result follows easily.

\section{The Hopf-Lax type formula in the CONVEX CASE}

In order to inject some versatility in the search of solutions to (1), we introduce a function $F: X^{*} \rightarrow$ $\mathbb{R} \cup\{+\infty\}$ which is comparable to $H$ if not $H$ itself and a function $G: X^{*} \rightarrow \mathbb{R} \cup\{+\infty\}$ which is meant to replace the Fenchel conjugate $g^{*}$ of $g$. We make the assumption that

$$
\operatorname{dom} F \cap \operatorname{dom} G \neq \emptyset
$$

where $\operatorname{dom} F:=F^{-1}(\mathbb{R})$ and $\operatorname{dom} G:=G^{-1}(\mathbb{R})$. It is satisfied if $F$ is everywhere finite and if $G:=g^{*}$, with $g$ bounded below by a continuous affine function and finite somewhere. In this section we deal with the Hopf-Lax type formula:

$$
v(x, t):=(G+t F)^{*}(x):=\sup _{p \in \operatorname{dom} F \cap \operatorname{dom} G}(p \cdot x-G(p)-t F(p)),
$$

for $(x, t) \in X \times \mathbb{R}_{+}$, where, for $t=0$, the product $t F(p)$ is interpreted as 0 if $F(p)<+\infty$, and $+\infty$ if $F(p)=+\infty$. We set $v(x, t)=+\infty$ for $(x, t) \in X \times(-\mathbb{P})$. This interpretation of formula (16) shows that $-\infty<v(\cdot, 0)=\left(G+\iota_{\operatorname{dom} F}\right)^{*}$ whenever (15) holds and that $v$ is the supremum of the continuous linear functions $(x, t) \mapsto p \cdot x-G(p)-\operatorname{tr}$ for $p \in \operatorname{dom} F \cap \operatorname{dom} G, r \geq F(p)$, so that the following lemma ensues, its last assertion being a consequence of the Brøndsted-Rockafellar [31] Theorem. Here $\iota_{\operatorname{dom} F}$ is the indicator function $\iota_{S}$ of $S:=\operatorname{dom} F$. Note that if assumption (15) is not satisfied the function $v$ is identically $-\infty$ on $X \times \mathbb{R}_{+}$, so that the following statements are trivial. 
Lemma 4.1. When assumption (15) is satisfied, the function $v$ is convex, lower semicontinuous (l.s.c.) and bounded below by the function $(x, t) \mapsto a \cdot x-G(a)-t F(a)$ for every $a \in \operatorname{dom} G \cap \operatorname{dom} F$. If moreover $\operatorname{dom} G^{*} \neq \emptyset$, or, more generally, $\operatorname{dom}\left(G+\iota_{\operatorname{dom} F}\right)^{*} \neq \emptyset$, then $v$ is a l.s.c. proper convex function of $(x, t)$ and, when $X$ is complete, there exists a dense subset $D$ of $\operatorname{dom} v$ containing $\operatorname{int}(\operatorname{dom} v)$ such that $\partial v(x, t) \neq \emptyset$ for each $(x, t) \in D$.

In the classical case $F=H, G=g^{*}, g$ is supposed to be Lipschitz and convex and $H$ is supposed to be finite and continuous ( $[12],[50],[51],[60],[76] \ldots)$ or boundedness and uniform continuity assumptions are made ( $[40],[37],[39] \ldots$.$) . The fact that H$ is not supposed to be finite everywhere allows to deal with cases such as $H(p)=-\sqrt{1-p^{2}}$ for $p \in[-1,1], H(p)=+\infty$ for $p \in \mathbb{R} \backslash[-1,1]$. Other examples follow; in them we assume $G=g^{*}, F=H$.

Example 5. Let $G:=g:=\iota_{\{a\}}, F:=H$. Then formula $(16)$ gives $v(x, t)=t H^{*}((x-a) / t)$ for $t>0$, $v(x, 0)=\iota_{\text {dom } H}^{*}(x-a)$.

Example 6. Let $g:=\min (\|\cdot\|, 1), H(p, q)=c\|p\|$. Then formula $(16)$ gives $v(x, t)=-t H(0)$.

These examples show that some assumptions are required in order that (16) gives a realistic solution to the system (1)-(2).

The proof of the first result we present is simpler than the ones given in [94] Cor. 12.9 and [104] Prop. 2.1; it is partly inspired by the proof of [4] Theorem 2.5 and the statement of [63] Prop. 1.2. Note that since we use the Fenchel conjugacy associated with the pairing between $X$ and $X^{*}$, the assumption $G=G^{* *}$ in the following statement means that $G$ is a convex function on $X^{*}$ which is l.s.c. for the weak* topology. It is satisfied when $G=g^{*}$, so that, with such a choice and $F=H$, no assumption is required (but (15) ensuring finiteness which is in force in all the present section).

Theorem 4.2. (a) Assume $F \leq H$ and $\left(G+\iota_{\operatorname{dom} F}\right)^{* *} \geq G$ (or, a fortiori, $G^{* *}=G$, what occurs when $\left.G=g^{*}\right)$. Then for any $(x, t) \in X \times \mathbb{P}$ and any $(p, q) \in \partial v(x, t)$ one has $q+H(p) \geq 0$ : the function $v$ given by (16) is a Hadamard supersolution to (1).

(b) Assume $F^{* *} \leq H$ and $(G+t F)^{* *}(p)=G^{* *}(p)+t F^{* *}(p) \in \mathbb{R}$ for some $t \in \mathbb{P}$ and some $p \in \operatorname{dom} F \cap \operatorname{dom} G$. Then for any $x \in X$ and any $q \in \mathbb{R}$ such that $(p, q) \in \partial v(x, t)$ one has $q+H(p) \geq 0$.

Proof. Let $(x, t) \in X \times \mathbb{P}$ and let $(p, q) \in \partial v(x, t)$. Since $v$ is convex, $\partial v(x, t)=\partial^{c} v(x, t)$, so that, for any $s \in \mathbb{R}_{+}, w \in X$ one has

(a) Then, for $s=0$, we get

$$
v(w, s) \geq v(x, t)+p \cdot(w-x)+q(s-t) .
$$

$$
\left(G+\iota_{\operatorname{dom} F}\right)^{*}(w)-p \cdot w+p \cdot x+q t \geq v(x, t)
$$

Taking the infimum on $w$ and using the assumption $\left(G+\iota_{\operatorname{dom} F}\right)^{* *} \geq G$ we get

$$
p . x-G(p)+q t \geq v(x, t) \geq p . x-G(p)-t F(p) .
$$

The first inequality shows that $G(p)<\infty$; it follows that $q+F(p) \geq 0$, hence $q+H(p) \geq 0$ when $H \geq F$.

(b) Observing that

we get from (17) with $s=0$

$$
(G+t F)^{* *}(p) \geq p . x-v(x, t)
$$

$$
v(w, 0)-p . w+(G+t F)^{* *}(p) \geq-q t .
$$

Using our assumption about $(G+t F)^{* *}(p)$ and taking the infimum over $w \in X$ we obtain

$$
-\left(G+\iota_{\operatorname{dom} F}\right)^{* *}(p)+G^{* *}(p)+t F^{* *}(p) \geq-q t
$$

Since $G^{* *}(p) \leq\left(G+\iota_{\operatorname{dom} F}\right)^{* *}(p) \leq\left(G+\iota_{\operatorname{dom} F}\right)(p)<\infty$ by assumption, we get $H(p) \geq F^{* *}(p) \geq-q$.

Under mild assumptions $v$ is a lower solution. 
Proposition 4.3. (a) Assume (15), $\operatorname{dom} G^{*} \neq \emptyset, F^{* *} \geq H$. Then for any $(x, t) \in X \times \mathbb{P}$ and any $(p, q) \in \partial v(x, t)$ one has $q+H(p) \leq 0$.

(b) Assume (15), $F \geq H$, and that, for every $s \in \mathbb{P}$ large enough, the function $G+s F$ is a $w^{*}$-l.s.c. proper convex function. Then for any $(x, t) \in X \times \mathbb{P}$ and any $(p, q) \in \partial v(x, t)$ one has $q+H(p) \leq 0: v$ is a Hadamard lower solution.

Proof. (a) Fixing $s \in \mathbb{P}$ and taking the supremum on $w \in X$ in (17) we get

$$
\begin{aligned}
p . x+q(t-s)-v(x, t) & \geq \sup _{w \in X}\left(p \cdot w-(G+s F)^{*}(w)\right)=(G+s F)^{* *}(p) \\
& \geq G^{* *}(p)+s F^{* *}(p) .
\end{aligned}
$$

It follows that $G^{* *}(p)<+\infty$; since $\operatorname{dom} G^{*} \neq \emptyset, G^{* *}(p)>-\infty$ and since $s$ can be arbitrarily large, we get $-q \geq F^{* *}(p)$. Thus, $q+H(p) \leq 0$ when $F^{* *} \geq H$.

(b) When $G+s F$ is a l.s.c. proper convex function, the right-hand side $(G+s F)^{* *}(p)$ of $(18)$ is $(G+s F)(p)$. Then $G(p)$ is finite and since $s$ can be arbitrarily large, we get $-q \geq F(p)$.

Gathering assertions a) of Theorem 4.2 and Proposition 4.3 we get the following result.

Corollary 4.4. Suppose $F=H=H^{* *}, G=g^{*}$ with $\operatorname{dom} g \neq \emptyset$ and (15) holds. Then $v$ given by (16) is a Hadamard solution i.e. a solution to (1) for the Hadamard subdifferential $\partial$ : for any $(x, t) \in X \times \mathbb{P}$ and any $(p, q) \in \partial v(x, t)$ one has $q+H(p)=0$.

In general, without convexity assumptions on $H$, the function $v$ is not a subsolution.

Example 7. ( $[4$, Remark 2.6]) Let $g:=\|\cdot\|, H:=-\|\cdot\|$. Then $v(x, t)=\|x\|+t$ and $(0,1) \in \partial v(0, t)$ but $1+H(0)>0$.

The following lemma, which will help to give conditions ensuring that $v$ is a subsolution, has an independent interest. Moreover, we observe that relation (19) below can be another interpretation of a notion of solution.

Lemma 4.5. Let $(x, t) \in X \times \mathbb{P}$ be such that the conjugate in the definition of $v$ is exact at $x$, i.e. there exists $\bar{p} \in \operatorname{dom} G \cap \operatorname{dom} F$ such that $\bar{p}$ is a maximizer of $\langle\cdot, x\rangle-G-t F$ and suppose $F(\bar{p})=H(\bar{p})$. Then

$$
(\bar{p},-H(\bar{p})) \in \partial v(x, t) .
$$

Moreover, for any $(p, q) \in \partial^{+} v(x, t):=-\partial(-v)(x, t)$ one has $q+H(p)=0$ and, when $\partial^{+} v(x, t)$ is nonempty, $v$ is Gâteaux-differentiable at $(x, t)$.

Proof. The assumption ensures that $v(x, t)=\langle\bar{p}, x\rangle-G(\bar{p})-t F(\bar{p})$, so that, for any $(w, s) \in X \times \mathbb{P}$ we have

$$
\begin{aligned}
v(w, s)-v(x, t) & \geq(\bar{p} \cdot w-G(\bar{p})-s F(\bar{p}))-(\bar{p} \cdot x-G(\bar{p})-t F(\bar{p})) \\
& \geq \bar{p} \cdot(w-x)-(s-t) F(\bar{p}) .
\end{aligned}
$$

Since $X \times \mathbb{P}$ is a neighborhood of $(x, t)$, this inequality is enough to ensure that $(\bar{p},-F(\bar{p})) \in \partial^{c} v(x, t)=\partial v(x, t)$. Thus, when $F(\bar{p})=H(\bar{p}),(\bar{p},-H(\bar{p})) \in \partial v(x, t)$. Moreover, the convexity of $v$ ensures that whenever $\partial(-v)(x, t)$ is nonempty, the function $v$ is Gâteaux-differentiable at $(x, t)$. Thus, one has $(p, q)=(\bar{p},-H(\bar{p}))$ for any $(p, q) \in-\partial(-v)(x, t)$ and $p=\bar{p}, q=-H(\bar{p})=-H(p)$.

Proposition 4.6. Suppose that for some $(x, t) \in X \times \mathbb{P}$ and some $\bar{p} \in \partial v(\cdot, t)(x)$ one has $G(\bar{p})=G^{* *}(\bar{p})$, $F(\bar{p})=H(\bar{p})=F^{* *}(\bar{p})$. Then $\bar{p}$ is a maximizer of $\langle\cdot, x\rangle-G-t F,(\bar{p},-H(\bar{p})) \in \partial v(x, t)$ and for any $q$ such that $(\bar{p}, q) \in \partial v(x, t)$ one has $q+H(\bar{p})=0$. Moreover for any $(p, q) \in-\partial(-v)(x, t)$, and any $(p, q) \in \partial v(x, t)$ one has $p=\bar{p}, q+H(p)=0$ and $v$ is Gâteaux-differentiable at $(x, t)$. 
Proof. The assumption $\bar{p} \in \partial v(\cdot, t)(x)$ is equivalent to $v(x, t)=\bar{p} \cdot x-(G+t F)^{* *}(\bar{p})$. Since for any function $f$ having a continuous affine minorant the greatest lower semicontinuous convex function majorized by $f$ is $f^{* *}$, we have

$$
(G+t F)(\bar{p}) \geq(G+t F)^{* *}(\bar{p}) \geq G^{* *}(\bar{p})+t F^{* *}(\bar{p})
$$

and, when $G(\bar{p})=G^{* *}(\bar{p}), F^{* *}(\bar{p})=F(\bar{p})=H(\bar{p})$, these relations are equalities. Then, we see that the definition of $v$ entails that $\bar{p}$ is a maximizer of $\langle\cdot, x\rangle-G-t F$ : for every $p \in X^{*}$

$$
\bar{p} . x-(G+t F)(\bar{p})=\bar{p} \cdot x-(G+t F)^{* *}(\bar{p})=v(x, t) \geq p \cdot x-(G+t F)(p) .
$$

The preceding lemma yields $(\bar{p},-H(\bar{p})) \in \partial v(x, t)$. Let $(\bar{p}, q) \in \partial v(x, t)$. Since $G(\bar{p})=G^{* *}(\bar{p})$ the proofs of Theorem 4.2 a) and Proposition 4.3 a) show that $q+H(\bar{p}) \geq 0$ and $q+H(\bar{p}) \leq 0$ respectively. The remaining conclusions follow from Proposition 4.2 and the preceding lemma.

Let us now deal with conditions ensuring that $v$ is a subsolution without assuming the convexity assumptions of the preceding proposition. We will use the following assumptions:

(A1) $g$ is bounded above on a ball centered at 0 in $X$ with radius $r>0$ and $G=g^{*}$;

(A1') $g$ is bounded above on bounded subsets of $X$ and $G=g^{*}$;

(A2) $F=H$ is weak* lower semicontinuous (l.s.c.) and $c:=\liminf _{\|p\| \rightarrow \infty} H(p) /\|p\|>-\infty$;

(A2') $F=H$ is weak* ${ }^{*}$ l.s.c. and hyper-coercive $(H(p) /\|p\| \rightarrow \infty$ as $\|p\| \rightarrow \infty)$.

Let us note that assumption (A1) holds whenever $g$ is upper semicontinuous and finite at 0 with $G=g^{*}$; its strengthening (A1') is satisfied whenever $X$ is finite dimensional, $G=g^{*}, g$ is convex (or convex up to a power of the norm, in particular, when $g$ is paraconvex or semiconvex) and finite on $X$. Assumption (A2) is satisfied whenever $H$ is weak* l.s.c. proper convex. However, neither (A1) nor (A2) involve an explicit convexity assumption. In particular, it is known that certain integral functionals are weakly lower semicontinuous without being convex ([32], [52], [53], [64], [65], [89]).

Theorem 4.7. Suppose assumptions (A1), (A2) are satisfied. Then for each $(x, t) \in X \times \mathbb{P}$ such that $\|x\|-t c<r$ and each $(p, q) \in \partial^{+} v(x, t):=-\partial(-v)(x, t)$ one has $q+H(p)=0$ and, if $\partial^{+} v(x, t)$ is nonempty, $v$ is Gâteauxdifferentiable at $(x, t)$. In particular, if (A1'), (A2) or if (A1), (A2') are satisfied, $v$ is a subsolution and an upper solution to (1).

Proof. Let $(x, t) \in X \times \mathbb{P}$ be such that $\|x\|-t c<r$. By the preceding lemma, it suffices to show that the conjugate in the definition of $v$ is exact at $(x, t)$. Let $c^{\prime} \in \mathbb{R}$ be such $c^{\prime}<c$ and $\|x\|-t c^{\prime}<r$. Let $s>0$ be such that $H(p) \geq c^{\prime}\|p\|$ for $p \in X^{*} \backslash s B_{X^{*}}$. Then, for each $p \in X^{*} \backslash s B_{X^{*}}$, setting $m:=\sup \left\{g(w): w \in r B_{X}\right\}$, we have

$$
\begin{aligned}
g^{*}(p)+t H(p) & \geq \sup _{w \in B(0, r)}(p \cdot w-m)+t c^{\prime}\|p\| \\
& \geq\left(r+t c^{\prime}\right)\|p\|-m
\end{aligned}
$$

hence

$$
-p \cdot x+g^{*}(p)+t H(p) \geq\left(r+t c^{\prime}-\|x\|\right)\|p\|-m
$$

Therefore the function $p \mapsto-p . x+g^{*}(p)+t H(p)$ is weak* l.s.c. and coercive. Thus it attains its infimum and Lemma 4.5 applies.

When (A1'), (A2) are satisfied, given an arbitrary pair $(x, t) \in X \times \mathbb{P}$, we choose $r>\|x\|-t c$ with $c$ given by (A2) and use the fact that $g$ is bounded above on $r B_{X}$. When (A1), (A2') are satisfied, given $(x, t) \in X \times \mathbb{P}$ and $r$ as in (A1), we pick $c^{\prime}>t^{-1}(\|x\|-r)$ and $s>0$ such that $H(p) \geq c^{\prime}\|p\|$ for $p \in X^{*} \backslash s B_{X^{*}}$ and the same estimates are valid.

When (A2) holds, $G=g^{*}$ and $g$ is bounded above on some ball $B(\bar{x}, r)$ the relation $q+H(p)=0$ holds for every $(p, q) \in \partial^{+} v(x, t)$ with $(x, t) \in X \times \mathbb{P}$ such that $\|x-\bar{x}\|-t c<r$. 


\section{The Hopf formulas in the QuASiconvex CASE}

In the present section, we suppose $H$ is defined on $X^{*} \times \mathbb{R}$ with values in $\overline{\mathbb{R}}$ and we use some functions $F$, $G: X^{*} \times \mathbb{R} \rightarrow \overline{\mathbb{R}}$ (and not just in $\mathbb{R} \cup\{+\infty\}$ ) as substitutes for $H$ and some conjugate of $g$ respectively. Again, we set $\operatorname{dom} H:=\left\{(p, q) \in X^{*} \times \mathbb{R}: H(p, q)<+\infty\right\}$ and we assume that

$$
F(X \times \mathbb{R}) \subset \mathbb{R} \cup\{+\infty\}, \quad \operatorname{dom} F \cap \operatorname{dom} G \neq \emptyset .
$$

We will consider the following functions as possible candidates to solutions of (1):

$$
\begin{aligned}
v_{\sharp}(x, t) & :=v_{b}(x, t):=(G+t F)^{\sharp}(x) \text { for }(x, t) \in X \times \mathbb{R}_{+}, \\
v_{\%}(x, t) & :=v_{\div}(x, t):=(G+t F)^{\%}(x) \text { for }(x, t) \in X \times \mathbb{R}_{+},
\end{aligned}
$$

with the convention that $(-\infty)+r=-\infty$ for any $r \in \overline{\mathbb{R}}$ and $0(-\infty)=-\infty, 0(+\infty)=+\infty$. Without assumption $(20)$, both $v_{\sharp}$ and $v_{\%}$ would be identically $-\infty$, a case without interest. Note that these formulas coincide with the ones obtained by using the dualities $b$ and $\div$ respectively, but since $G$ can stand for $g^{b}$ or $g^{\div}$instead of $g^{\sharp}$ and $g^{\%}$ respectively, four cases are at hand; the use of $G$ allows to reduce them to two cases. The following lemma justifies our choice of the value of $v(\cdot, 0)$.

Lemma 5.1. For any functions $F, G$, the functions $v$ defined by the preceding formulas are quasiconvex and lower semicontinuous in $(x, t)$.

Proof. Let us consider the case of the musical duality, the other case being similar. Let $r, r^{\prime} \in[0,1]$, with $r+r^{\prime}=1, x, x^{\prime} \in X, t, t^{\prime} \in \mathbb{P}$ and let $x^{\prime \prime}:=r x+r^{\prime} x^{\prime}, t^{\prime \prime}:=r t+r^{\prime} t^{\prime}$. Suppose $v\left(x^{\prime \prime}, t^{\prime \prime}\right)>m:=$ $\max \left(v(x, t), v\left(x^{\prime}, t^{\prime}\right)\right)$. By definition of the sharp quasi-duality, there exists $q>m$ such that $\left(G_{q}+t^{\prime \prime} F_{q}\right)^{*}\left(x^{\prime \prime}\right)>0$. Thus, there exists $p \in X^{*}$ such that

$$
p . x^{\prime \prime}-G(p, q)-t^{\prime \prime} F(p, q)>0 .
$$

However, the choice of $m$ ensures that

$$
\begin{aligned}
p . x-G(p, q)-t F(p, q) & \leq 0, \\
p . x^{\prime}-G(p, q)-t^{\prime} F(p, q) & \leq 0 ;
\end{aligned}
$$

multiplying both sides of these relations by $r$ and $r^{\prime}$ respectively and summing we get a contradiction. When $t^{\prime}=0$, the last relation has to be replaced with

$$
p . x^{\prime}-G(p, q) \leq 0, \quad F(p, q)<\infty,
$$

and we get again a contradiction since in that case $t^{\prime \prime}=r t$. The lower semicontinuity of $v$ stems from the fact that when $v\left(x_{0}, t_{0}\right)>m$ with $\left(x_{0}, t_{0}\right) \in X \times \mathbb{P}$ there exist $q>m$ and $p \in X^{*}$ such that

$$
p . x_{0}-G(p, q)-t_{0} F(p, q)>0 .
$$

For $t_{0}>0$, we have $F(p, q)<\infty$, so that for $(x, t) \in X \times \mathbb{P}$ close enough to $\left(x_{0}, t_{0}\right)$ we still have $p . x-G(p, q)-$ $t F(p, q)>0$. When $t_{0}=0$, relation (23) has to be replaced by $p . x_{0}-G(p, q)>0, F(p, q)<\infty$ and again $p . x-G(p, q)-t F(p, q)>0$ for $(x, t) \in X \times \mathbb{R}_{+}$close enough to $\left(x_{0}, t_{0}\right)$.

Proposition 5.2. Suppose $F \leq H, G^{\% \%} \geq G$ (resp. $G^{\div \div} \geq G$ ), assumption (20) holds and for each $t>0$ the function $K:=G+t F$ is right lower regular. Then the function $v:=v_{\%}:=v_{\div}$given by formula (22) is a supersolution to (1) for the infradifferential $\partial \leq$ (resp. the lower subdifferential $\left.\partial^{<}\right)$: for any $(x, t) \in X \times \mathbb{P}$ and any $(p, q) \in \partial^{\leq} v(x, t)\left(\right.$ resp. $\left.(p, q) \in \partial^{<} v(x, t)\right)$ one has $q+H(p, v(x, t)) \geq 0$. 
Proof. We first consider the case $v=v_{\%}$ as given by $(22)$. Let $(x, t) \in X \times \mathbb{P}, r:=v(x, t)$ and $(p, q) \in$ $\partial \leq v(x, t)$; assuming that $G^{\% \%}(p, r) \geq G(p, r)$ we will prove that $q+F(p, r) \geq 0$, hence $q+H(p, r) \geq 0$ when $F \leq H$. Suppose on the contrary that $\varepsilon:=-(q+F(p, r))>0$. For any $z \in X$ such that $G^{\%}(z) \leq r$ Lemma 2.5 ensures that $v(z, 0):=\left(G+\iota_{\operatorname{dom} F}\right)^{\%}(z) \leq G^{\%}(z) \leq r$ hence, by the definition of the infradifferential,

$$
G^{\%}(z)-p . z \geq v(z, 0)-p . z \geq v(x, t)-p . x-q t .
$$

Taking the infimum over $z \in\left[G^{\%} \leq r\right]$, we get

$$
-G^{\% \%}(p, r) \geq v(x, t)-p . x-q t .
$$

Since $G^{\% \%}(p, r) \geq G(p, r)$ it follows that

$$
p . x-G(p, r)-t F(p, r) \geq r-q t-t F(p, r)=r+t \varepsilon .
$$

Our right regularity assumption ensures that there exist sequences $\left(p_{n}\right) \rightarrow p,\left(r_{n}\right) \rightarrow r_{+}$such that

$$
p . x-G\left(p_{n}, r_{n}\right)-t F\left(p_{n}, r_{n}\right)>r+t \varepsilon / 2
$$

hence

$$
p_{n} . x-G\left(p_{n}, r_{n}\right)-t F\left(p_{n}, r_{n}\right)>r_{n}
$$

for $n$ large enough. Therefore, we get

$$
v(x, t) \geq r_{n} \wedge\left(G\left(\cdot, r_{n}\right)+t F\left(\cdot, r_{n}\right)\right)^{*}(x) \geq r_{n} \wedge\left(p_{n} . x-G\left(p_{n}, r_{n}\right)-t F\left(p_{n}, r_{n}\right)\right)>r
$$

a contradiction.

For the case $v=v_{\div}$, we take $z \in\left[G^{\div}<r\right]$, where $r:=v(x, t)$, so that taking the infimum on $z$, similarly to inequality (25), we get

$$
-G^{\div \div}(p, r) \geq v(x, t)-p \cdot x-q t
$$

and obtain again a contradiction to the assumption $\varepsilon:=-(q+F(p, r))>0$.

The following result is more interesting as it involves the Hadamard subdifferential.

Theorem 5.3. Suppose $F \leq H, G^{\sharp \sharp} \geq G$ (resp. $G^{b b} \geq G$ ), assumption (20) holds and for each $t>0$ the function $K:=G+t F$ is right lower regular, or more generally, that $K_{q+0}^{*} \geq K_{q}^{*}$ for every $q \in \mathbb{R}$. Then the function $v:=v_{\sharp}$ given by formula (21) is a supersolution to (1) for the normal subdifferential $\partial^{\nu}$ (resp. the star subdifferential $\left.\partial^{\circledast}\right)$, hence is a Hadamard supersolution.

Proof. Let $(x, t) \in X \times \mathbb{P}, r:=v(x, t)$ and $(p, q) \in \partial^{\nu} v(x, t)$. For any $z \in\left[G^{\sharp} \leq r\right]$ one has $v(z, 0) \leq$ $G^{\sharp}(z) \leq r$, hence, by the definition of $\partial^{\nu}$ and the fact that $v$ is quasiconvex, one gets $p \cdot(z-x)-q t \leq 0$, hence $G(p, r) \leq G^{\sharp \sharp}(p, r) \leq q t+p . x$. Since $K_{r+0}^{*} \geq K_{r}^{*}$ and $K_{r+0}^{*}(x) \leq 0$, one has $K_{r}^{*}(x) \leq 0$, hence $G(p, r)+t F(p, r) \geq p . x$. Then, the estimate $q t+p . x \geq G(p, r)$ yields $q+F(p, r) \geq 0$. In the case $(p, q) \in \partial^{\circledast} v(x, t)$, for any $z \in\left[G^{b}<r\right]$ one has $v(z, 0) \leq G^{b}(z)<r$, hence, by the definition of $\partial^{\circledast}$ one gets $p .(z-x)-q t \leq 0$. and $G(p, r) \leq G^{b b}(p, r) \leq q t+p . x$. Since $K_{r+0}^{*} \geq K_{r}^{*}$ and $K_{r+0}^{*}(x) \leq 0$, one has $K_{r}^{*}(x) \leq 0$, hence again $G(p, r)+t F(p, r) \geq p . x$. Then, the estimate $q t+p . x \geq G(p, r)$ yields $q+F(p, r) \geq 0$.

The next result can serve as a criteria for the assumptions of the preceding theorem; here we say that $g$ is inf-compact if for every $r \in \mathbb{R}$ the sublevel set $[g \leq r]$ is compact.

Lemma 5.4. Suppose $g$ is inf-compact for (a topology $\mathcal{T}$ on $X$ which is at least as strong as) the weak topology. Then, the function $G:=g^{\sharp}$ (resp. $G:=g^{\%}$ ) is upper semicontinuous (u.s.c.) and for each $p \in X^{*}$ the function $G(p, \cdot)$ is nondecreasing. Moreover one has $G^{\sharp \sharp}=G$. In particular these conclusions hold when $X$ is reflexive and $g$ is a l.s.c. coercive quasiconvex function. 
Proof. The function $g^{\sharp}(p, \cdot)$ is clearly nondecreasing. Suppose $g^{\sharp}$ is not u.s.c. at some $(p, q) \in X \times \mathbb{R}$. Then there exist some real number $r>g^{\sharp}(p, q)$ and some sequence $\left(\left(p_{n}, q_{n}\right)\right)$ with limit $(p, q)$ such that $g^{\sharp}\left(p_{n}, q_{n}\right)>r$ for each $n$. Let $x_{n} \in\left[g \leq q_{n}\right]$ be such that $p_{n} . x_{n} \geq r$. Since $\left(x_{n}\right)$ is contained in a compact set for the topology $\mathcal{T}$, the sequence $\left(x_{n}\right)$ has a cluster point $x_{\infty}$. Since $g$ is l.s.c. for $\mathcal{T}$, one has $g\left(x_{\infty}\right) \leq q$. Moreover, $\mathcal{T}$ being stronger than the weak topology, we have $p . x_{\infty} \geq r$, a contradiction with $r>g^{\sharp}(p, q)$. The proof for $g^{\%}$ is similar : we take $x_{n} \in\left[g \leq q_{n}\right]$ such that $p_{n} \cdot x_{n}-g\left(x_{n}\right) \geq r$ and we get $p . x_{\infty}-g\left(x_{\infty}\right) \geq r$, a contradiction with $r>g^{\%}(p, q)$ and $x_{\infty} \in[g \leq q]$.

The relation $G^{\sharp \sharp}=G\left(\right.$ resp. $\left.G^{\% \%}=G\right)$ stems from Lemma 2.6(c).

Using the fact that the sum of a right lower regular function and of an u.s.c. function is right lower regular, we deduce from Theorem 5.3 and the preceding lemma more handable criteria.

Corollary 5.5. Let $F \leq H, G:=g^{\sharp}$ where $g$ is inf-compact for the weak topology and let $F$ be right lower regular. Then $v_{\sharp}$ given by (21) is a supersolution to (1) for the normal subdifferential $\partial^{\nu}$.

Using the same argument and Proposition 2.16 in order to have $G^{\sharp \sharp}=G$ when $G=g^{\sharp}$, we obtain another consequence.

Corollary 5.6. Suppose $X$ is reflexive, $g$ is an arbitrary function, $G:=g^{\sharp}$ and $F=H$ is nondecreasing in its second variable and jointly u.s.c. at each point of $\operatorname{dom} G$. Then the function $v_{\sharp}$ given by (21) is a (normal) supersolution to (1).

Now let us examine conditions ensuring $v:=v_{b}=v_{\sharp}$ is a subsolution.

Proposition 5.7. Suppose $F^{\mathrm{bb}} \geq H$ and that $F$ and $G$ are nondecreasing functions of their second variable. Then for any $(x, t) \in X \times \mathbb{P}$ and for any $(p, q) \in-\partial^{\nu}(-v)(x, t)$ one has $q+H(p, v(x, t)) \leq 0$.

Proof. Let $r:=v(x, t)$. In view of the inequality $F^{b b} \geq H$ it suffices to show that

$$
p . z \leq-q \quad \forall z \in\left[F^{b}<r\right] .
$$

Let $z \in\left[F^{b}<r\right]$. The definition of $F^{b}$ ensures that for any $s>F^{b}(z)$ one has $\left(F_{s}\right)^{*}(z) \leq 0$. Similarly, the definition of $(G+t F)^{b}$ entails that for any $\left.r^{\prime} \in\right] F^{b}(z), r\left[\right.$ there exists $s \in\left[r^{\prime}, r\right]$ such that $\left(G_{s}+t F_{s}\right)^{*}(x)>0$. Thus, there exists some $p^{\prime} \in X^{*}$ such that

$$
p^{\prime} . x-G\left(p^{\prime}, s\right)-t F\left(p^{\prime}, s\right)>0 .
$$

Since $\left(F_{s}\right)^{*}(z) \leq 0$ we have $p^{\prime} . z-F\left(p^{\prime}, s\right) \leq 0$. Combining this inequality with the preceding one, we get, for every $\left.t^{\prime} \in\right] 0, t[$,

$$
p^{\prime} .\left(x-t^{\prime} z\right)-G\left(p^{\prime}, s\right)-\left(t-t^{\prime}\right) F\left(p^{\prime}, s\right)>0,
$$

hence $\left(G_{s}+\left(t-t^{\prime}\right) F_{s}\right)^{*}\left(x-t^{\prime} z\right)>0$. Therefore, $v\left(x-t^{\prime} z, t-t^{\prime}\right) \geq s \geq r^{\prime}$ for any $\left.r^{\prime} \in\right] F^{b}(z), r[$; it follows that $v\left(x-t^{\prime} z, t-t^{\prime}\right) \geq r$. Since this inequality is valid for any $\left.t^{\prime} \in\right] 0, t\left[\right.$ and since $(-p,-q) \in N^{r}([v \geq r],(x, t))$, we get $(-p) \cdot(-z)+(-q)(-1) \leq 0:(26)$ is established.

A similar result holds for solution obtained via the $\div$ duality.

Proposition 5.8. Let $v:=v_{\div}$. Suppose $F \div \div \quad \geq H$ and that $F$ and $G$ are nondecreasing functions of their second variable. Then $v$ is a subsolution: for any $(x, t) \in X \times \mathbb{P}$ and for any $(p, q) \in-\partial(-v)(x, t)$ one has $q+H(p, v(x, t)) \leq 0$.

Proof. Let $r:=v(x, t)$ and $(p, q) \in-\partial(-v)(x, t)$. Then $r$ is finite. In view of the inequality $F \div \div \geq H$ it suffices to show that

$$
p . z-F \div(z) \leq-q \quad \forall z \in[F \div<r] .
$$


Let $z \in[F \div<r]$ and let $r_{z}:=F \div(z)$. Let $\left(t_{n}\right)$ be a sequence in $\left.\left.] 0,1\right] \cap\right] 0, r-r_{z}\left[\right.$ with limit 0 and let $r_{n}:=r-t_{n}^{2}$. The definition of $(G+t F) \div$ entails that there exists $\left.s_{n} \in\right] r_{n}, r\left[\right.$ such that $\left(G_{s_{n}}+t F_{s_{n}}\right)^{*}(x)>r_{n}$. Thus, there exists some $p_{n} \in X^{*}$ such that

$$
p_{n} . x-G\left(p_{n}, s_{n}\right)-t F\left(p_{n}, s_{n}\right)>r_{n} .
$$

Since $s_{n}>r_{z}:=F \div(z)$, the definition of $F \div$ ensures that we have $\left(F_{s_{n}}\right)^{*}(z) \leq r_{z}$, hence $p_{n} . z-F\left(p_{n}, s_{n}\right) \leq r_{z}$. Combining this inequality with the preceding one, we get

$$
p_{n} \cdot\left(x-t_{n} z\right)-G\left(p_{n}, s_{n}\right)-\left(t-t_{n}\right) F\left(p_{n}, s_{n}\right)>r_{n}-t_{n} r_{z}
$$

hence $\left(G_{s_{n}}+\left(t-t_{n}\right) F_{s_{n}}\right)^{*}\left(x-t_{n} z\right)>r_{n}-t_{n} r_{z}$. Thus we see that $v\left(x-t_{n} z, t-t_{n}\right) \geq s_{n} \wedge\left(r_{n}-t_{n} r_{z}\right) \geq$ $\left(r-t_{n}^{2}\right) \wedge\left(r-t_{n}^{2}-t_{n} r_{z}\right)$. Since $(-p,-q) \in \partial(-v)(x, t)$, we get

$$
\begin{aligned}
(-p) \cdot(-z)+(-q)(-1) & \leq \liminf _{n \rightarrow \infty}\left(1 / t_{n}\right)\left[(-v)\left(x-t_{n} z, t-t_{n}\right)-(-v)(x, t)\right] \\
& \leq \liminf _{n \rightarrow \infty}\left(1 / t_{n}\right)\left[\left(-r+t_{n}^{2}\right) \vee\left(-r+t_{n}^{2}+t_{n} r_{z}\right)+r\right] \leq r_{z} .
\end{aligned}
$$

Therefore (27) is proved.

In the examples which follow, we take $F=H$ and $G=g^{\sharp}$ (resp. $G=g^{\%}$ ) when using formula (21) (resp. formula (22)).

Example 8. Let $g:=\iota_{\{a\}}$. Then formula (21) yields $v_{\sharp}(x, t)=H^{\sharp}((x-a) / t)$ and formula $(22)$ gives $v_{\%}(x, t)=$ $\max \left(H^{\sharp}((x-a) / t), 0\right)$.

Example 9. Let $g:=\min (\|\cdot\|, 1), H(p, q)=c\|p\|$. Then formulae (21), (22) yield $v(x, t)=\min \left((\|x\|-c t)_{+}, 1\right)$.

Example 10. Let $g:=\ln \|\cdot\|-c, H(p, q)=e^{q}\|p\|$. Then formula (21) yields $v(x, t)=\ln \|x\|-\ln \left(t+e^{c}\right)$.

\section{The LaX-Oleinik solution in the CONVEX CASE}

In the present section, given $g: X \rightarrow \overline{\mathbb{R}}$ and $H: X^{*} \rightarrow \overline{\mathbb{R}}$, we consider a Lax-Oleinik type formula defined with the help of the classical infimal convolution operator $\square$ given for $f, g \in \overline{\mathbb{R}}^{X}, x \in X$ by

$$
(f \square g)(x):=\inf \{f(u)+g(v): u, v \in X, u+v=x\} .
$$

Here we use a kind of "preconjugate" $h$ of $H$, i.e. a function $h$ on $X$ whose conjugate $h^{*}$ can be compared with $H$. Namely, we set for $(x, t) \in X \times \mathbb{P}$

$$
u(x, t):=\left(g \square h_{t}\right)(x):=\inf _{w \in X}\left(g(x-w)+h_{t}(w)\right),
$$

where $h_{t}:=t h\left(t^{-1}\right)$, so that $h_{t}=(t H)^{*}$ when $h=H^{*}$ (considered as a function on $X$ ), the usual case. We also assume that

$$
\operatorname{dom} g \neq \emptyset, \quad \operatorname{dom} h \neq \emptyset
$$

The assumption $\operatorname{dom} h \neq \emptyset$ is satisfied in particular when $H(\cdot) \geq\langle\cdot, a\rangle-b$ for some $a \in X, b \in \mathbb{R}$ and $h \leq H^{*}$; if $h=H^{*}$ it is equivalent to the later condition. In the classical case, $H$ is supposed to be convex (or concave), $h=H^{*}$ and $g$ is supposed to be Lipschitz, or $H$ is supposed to be Lipschitz, convex and $g$ is supposed to be continuous ( [60], [75], [50], [12], [122] ...).

When $h^{*} \leq F$ (in particular when $\left.h=H^{*}, H \leq F\right), g^{*} \leq G$ we observe that since $g^{*}+t h^{*} \leq G+t F$, we have

In fact

$$
u(\cdot, t)=g \square h_{t} \geq\left(g \square h_{t}\right)^{* *}=\left(g^{*}+t h^{*}\right)^{*} \geq(G+t F)^{*}=v(\cdot, t) .
$$

$$
(u(\cdot, t))^{* *}=\left(g \square h_{t}\right)^{* *}=\left(g^{*}+t h^{*}\right)^{*} \geq(G+t F)^{*}=v(\cdot, t),
$$

with equality when $G=g^{*}, F=h^{*}$. We will make use of the following fact. 
Lemma 6.1. For any $r, s>0$ and any function $h$ one has $h_{r} \square h_{s} \leq h_{r+s}$. When $h$ is convex one has the semi-group relation

$$
h_{r} \square h_{s}=h_{r+s} .
$$

When $g$ and $h$ are both convex, $u$ is convex.

Proof. For $x \in X, t:=r+s$ one has

$$
\begin{aligned}
h_{t}(x) & =\operatorname{th}\left(t^{-1} x\right)=r h\left(r^{-1} r t^{-1} x\right)+s h\left(s^{-1} s t^{-1} x\right) \\
& =h_{r}\left(r t^{-1} x\right)+h_{s}\left(s t^{-1} x\right) \geq\left(h_{r} \square h_{s}\right)(x) .
\end{aligned}
$$

Moreover, when $h$ is convex one observes that

$$
\begin{aligned}
\left(h_{r} \square h_{s}\right)(x) & \geq t \inf _{w \in X}\left(t^{-1} r h\left(r^{-1} w\right)+t^{-1} \operatorname{sh}\left(s^{-1}(x-w)\right)\right) \\
& \geq t \inf _{w \in X} h\left(t^{-1} w+t^{-1}(x-w)\right)=h_{t}(x) .
\end{aligned}
$$

When $g$ and $h$ are convex, $u$ is convex as the performance function of the function $(w, x, t) \mapsto g(x-w)+$ $\operatorname{th}\left(t^{-1} w\right)$ in view of the relation

$$
r^{\prime} t^{\prime} h\left(\frac{x^{\prime}}{t^{\prime}}\right)+r^{\prime \prime} t^{\prime \prime} h\left(\frac{x^{\prime \prime}}{t^{\prime \prime}}\right) \geq \operatorname{th}\left(\frac{1}{t}\left(r^{\prime} x^{\prime}+r^{\prime \prime} x^{\prime \prime}\right)\right)
$$

valid for $r^{\prime}, r^{\prime \prime} \geq 0, r^{\prime}+r^{\prime \prime}=1, t:=r^{\prime} t^{\prime}+r^{\prime \prime} t^{\prime \prime}$. It follows that $\partial u(x, t)=\partial^{c} u(x, t)$.

In the absence of growth conditions, the function $u$ may take the value $-\infty$.

Example 11. ( [4, Lemma 5.3]) Let $X$ be a Hilbert space and let $g:=-(1 / 2)\|\cdot\|^{2}, h=H^{*}$ with $H:=(1 / 2)\|\cdot\|^{2}$. Then for $(x, t) \in X \times] 0,1\left[\right.$ one has $u(x, t)=-\|x\|^{2} / 2(1-t)$ and $u(0,1)=0, u(x, 1)=-\infty$ for $x \in X \backslash\{0\}$.

Let us note that the estimate (30) implies that $u$ does not take the value $-\infty$ when condition (15) holds with $F \geq h^{*}, G \geq g^{*}$. This condition can be rewritten

$$
\exists(\bar{p}, \bar{q}) \in X^{*} \times \mathbb{R}: \forall x \in X \quad h(x) \geq \bar{p} \cdot x+\bar{q}, g(x) \geq \bar{p} \cdot x+\bar{q} .
$$

We will impose stronger growth conditions.

In the following statement, we say that a function $k: X \rightarrow \overline{\mathbb{R}}$ is quasicoercive if there exists a bounded subset $B$ of $X$ such that inf $k(B)=\inf k(X)$. This condition is obviously satisfied if $k$ is coercive (i.e. $\lim _{\|x\| \rightarrow \infty} k(x)=$ $+\infty)$ or if $k$ attains its infimum or if $k$ is semicoercive in the sense of [92], i.e. $\liminf _{\|x\| \rightarrow \infty} k(x)>\inf k(X)$. In order to get a coercivity property, we will make the following assumption:

(C) $\liminf _{\|x\| \rightarrow \infty} h(x) /\|x\|>-\liminf _{\|x\| \rightarrow \infty} g(x) /\|x\|$.

This assumption is satisfied whenever one of the following conditions holds:

(C') there exists $b \in \mathbb{R}_{+}, b>-\liminf _{\|x\| \rightarrow \infty} g(x) /\|x\|$ such that $h^{*}$ is bounded above on the ball $B(0, b)$ of center 0 and radius $b$;

(C") $h \geq H^{*}$ with $H$ bounded above on $B(0, b)$ and $g$ is bounded below by $d-c\|\cdot\|$ for some $c<b$ and some $d \in \mathbb{R}$.

This assertion is a consequence of the following lemma which implies [36, Prop. 2.4] when $h$ is bounded below by an affine function.

Lemma 6.2. For any function $h$ on $X$ and for any $a \in \mathbb{R}, b \in \mathbb{R}_{+}$the following assertions are equivalent:

a) $h(x) \geq b\|x\|-a$ for each $x \in X$;

b) $h^{*}(p) \leq$ a for each $p \in B(0, b)$;

c) $h^{* *}(x) \geq b\|x\|-a$ for each $x \in X$. 
Proof. If a) holds, for each $p \in B(0, b)$ we have $h^{*}(p) \leq \sup \{p \cdot x-b\|x\|+a: x \in X\} \leq a$ since $p \cdot x \leq b\|x\|$. If b) holds, for each $x \in X$ we have $h^{* *}(x) \geq \sup \{p \cdot x-a: p \in B(0, b)\}=b\|x\|-a$. Clearly, c) implies a).

Thus, if (C') holds, setting $a:=\sup h^{*}(B(0, b))$ we have $\liminf _{\|x\| \rightarrow \infty} h(x) /\|x\| \geq b$ and (C) is satisfied. Since $h^{*} \leq H$ when $h \geq H^{*}$ condition (C") implies condition (C').

Now we observe that, whenever $(\mathrm{C})$ is satisfied, for every $(x, t) \in X \times \mathbb{P}$ the function $k: w \mapsto g(x-w)+$ $\operatorname{th}\left(t^{-1} w\right)$ is coercive since, for some $c, c^{\prime} \in \mathbb{R}$ with $c+c^{\prime}>0$, we have, for $\|w\|$ large enough, $g(x-w) \geq$ $c\|w\|-|c|\|x\|, \operatorname{th}\left(t^{-1} w\right) \geq c^{\prime}\|w\|$.

Theorem 6.3. Suppose $h^{*} \leq H$ (or, a fortiori, that $\left.h=H^{*}\right)$.

(a) If for some $(x, t) \in X \times \mathbb{P}$ the function $k: w \mapsto g(x-w)+t h\left(t^{-1} w\right)$ is quasicoercive then for every $(p, q) \in \partial^{-} u(x, t)$ one has $q+H(p) \geq 0$. In particular, when $h^{*} \leq H$ and $(C)$ holds, $u$ is a Fréchet supersolution.

(b) If the inf-convolution in the definition of $u$ is exact at $(x, t) \in X \times \mathbb{P}$, then for every $(p, q) \in \partial u(x, t)$ one has $q+H(p) \geq 0$. Under assumption $(C)$, the exactness of the inf-convolution occurs whenever $X$ is a dual space and $g$ and $h$ are weak ${ }^{*}$ l.s.c.; then $u$ is a Hadamard supersolution.

(c) If $g$ and $h$ are convex, then $u$ is a Hadamard supersolution.

Proof. (a) Let $B$ be a bounded subset of $X$ such that inf $k(B)=\inf k(X)$, with $k(w)=g(x-w)+t h\left(t^{-1} w\right)$. For each $s \in] 0, t\left[\right.$ let us pick $z_{s} \in B$ such that $k\left(z_{s}\right)<\inf k(X)+s^{2}=u(x, t)+s^{2}$. Then, we have

$$
\begin{aligned}
u\left(x-s t^{-1} z_{s}, t-s\right) & =\left(h_{t-s} \square g\right)\left(x-s t^{-1} z_{s}\right) \\
& \leq h_{t-s}\left(z_{s}-s t^{-1} z_{s}\right)+g\left(x-z_{s}\right) \\
& \leq(t-s) h\left(t^{-1} z_{s}\right)+g\left(x-z_{s}\right) \\
& \leq u(x, t)-\operatorname{sh}\left(t^{-1} z_{s}\right)+s^{2} .
\end{aligned}
$$

It follows that for each $(p, q) \in \partial^{-} u(x, t)$ one can find a function $\varepsilon: \mathbb{R}_{+} \rightarrow \mathbb{R}_{+}$with limit 0 at 0 such that

$$
-p . s t^{-1} z_{s}-s q-\varepsilon(s) s \leq u\left(x-s t^{-1} z_{s}, t-s\right)-u(x, t) \leq s^{2}-s h\left(t^{-1} z_{s}\right) .
$$

Therefore, when $H \geq h^{*}$ we obtain

$$
q+H(p) \geq q+p \cdot t^{-1} z_{s}-h\left(t^{-1} z_{s}\right) \geq-s-\varepsilon(s) .
$$

Taking the limit as $s \rightarrow 0_{+}$, we get $q+H(p) \geq 0$.

(b) When the inf-convolution in the definition of $u$ is exact at $(x, t)$ we can take for $B$ a singleton $\{z\}$ of $X$ and the same calculation can be performed with $(p, q) \in \partial u(x, t)$. The second assertion is a consequence of the fact that, under its assumptions, the function $k: w \mapsto g(x-w)+t h\left(t^{-1} w\right)$ is weakly* l.s.c. and coercive.

(c) When $g$ and $h$ are convex, $u$ is convex and $\partial u(x, t)$ coincides with the Fenchel subdifferential $\partial^{c} u(x, t)$ : given $(p, q) \in \partial u(x, t)$, for any $(w, s) \in X \times \mathbb{P}$ the following inequality holds

$$
u(w, s) \geq u(x, t)+\langle p, w-x\rangle+q(s-t) .
$$

Thus, for any $w, z \in X, s>0$, we have

$$
g(w-z)+h_{s}(z) \geq u(x, t)+\langle p, w-z\rangle+\langle p, z\rangle-\langle p, x\rangle+q(s-t),
$$

hence, rearranging terms, taking suprema on $w^{\prime}:=w-z$ and then on $z$, we get

$$
-s h^{*}(p)=-h_{s}^{*}(p) \geq u(x, t)+g^{*}(p)-\langle p, x\rangle+q(s-t) .
$$

Since (29) ensures that there exists some $z \in X$ such that $h_{s}(z)<\infty$, hence $h_{s}^{*}(p)>-\infty$, we deduce from (34) that $g^{*}(p)<\infty$; moreover, since the domain of $g$ is nonempty, $g^{*}(p)>-\infty$ and $g^{*}(p)$ is finite. Since 
$-h_{s}^{*}(p)<+\infty$ by $(29)$, it also follows from (34) that $h_{s}^{*}(p)=s h^{*}(p)$ is finite. Since $s$ can be arbitrarily close to 0 , and since by (30) and $h^{*} \leq H$ we have $u(x, t) \geq\left(g^{*}+t H\right)^{*}(x)$, we deduce from (34) that

$$
\begin{aligned}
q t+\langle p, x\rangle & \geq u(x, t)+g^{*}(p) \\
& \geq \sup _{p^{\prime} \in X^{*}}\left(\left\langle p^{\prime}, x\right\rangle-g^{*}\left(p^{\prime}\right)-t H\left(p^{\prime}\right)\right)+g^{*}(p) \\
& \geq\langle p, x\rangle-t H(p),
\end{aligned}
$$

hence $q+H(p) \geq 0$.

Remark. The proof of assertion c) shows that for any $(x, t) \in X \times \mathbb{P}$ and for any $(p, q) \in \partial^{c} u(x, t)$, one has $q+H(p) \geq 0$ whatever $g$ and $h$ are, provided $h^{*} \leq H$.

Corollary 6.4. Suppose $X$ is finite dimensional, $h^{*} \leq H$ and $(C)$ holds. Then $u$ is a Hadamard supersolution.

When $h^{*} \geq H$, since $h_{s}^{*}(p)=s h^{*}(p) \geq s H(p)$, and since $s$ can be arbitrarily large in relation (34), we get $q+h^{*}(p) \leq 0$, hence $q+H(p) \leq 0$. In the following statement we obtain that conclusion without assuming $g$ is convex or that $\partial^{c} u$ is substituted to $\partial u$.

Theorem 6.5. Suppose $h$ is convex and $h^{*} \geq H$. Then $u$ given by (28) is a Hadamard lower solution and a Hadamard subsolution.

Since in the Lax formula $H$ enters through its conjugate when we take $h=H^{*}, u$ is also determined by $H^{* *}$ in that case. Therefore, assuming that $H=H^{* *}$ is not a restrictive assumption when one takes $h=H^{*}$.

Proof. Let $(x, t) \in X \times \mathbb{R}$ and let $(p, q) \in \partial u(x, t)$. For any $s>0$ and $w \in X$, by relation (32) and the associativity of the infimal convolution we have

$$
\begin{aligned}
u(x+s w, t+s) & =\left(\left(g \square h_{t}\right) \square h_{s}\right)(x+s w) \\
& \leq u(x, t)+\operatorname{sh}(w) .
\end{aligned}
$$

It follows that

$$
\limsup _{s \rightarrow 0_{+}} \frac{1}{s}(u(x+s w, t+s)-u(x, t)) \leq h(w) .
$$

Thus

and we get

$$
\langle p, w\rangle+q 1 \leq h(w)
$$

$$
q+H(p) \leq q+\sup _{w \in X}(\langle p, w\rangle-h(w)) \leq 0 .
$$

Now let us show that $q+H(p) \leq 0$ for any $(p, q) \in-\partial(-u)(x, t)$. Since $H \leq h^{*}$, it suffices to prove that $\langle p, w\rangle-h(w) \leq-q$ for every $w \in X$. For each $s \in] 0, t[$, relation (32) yields

$$
\begin{aligned}
u(x, t) & =\left(\left(g \square h_{t-s}\right) \square h_{s}\right)(x) \\
& \leq\left(g \square h_{t-s}\right)(x-s w)+s h(w),
\end{aligned}
$$

so that

$$
s^{-1}((-u)(x-s w, t-s)-(-u)(x, t)) \leq h(w) .
$$

Taking the limit inferior we obtain

$$
\langle-p,-w\rangle+(-q)(-1) \leq \liminf _{s \rightarrow 0_{+}} s^{-1}((-u)(x-s w, t-s)-(-u)(x, t)) \leq h(w),
$$

as expected. 
Remark. The first part of the preceding proof shows that the relation $q+H(p) \leq 0$ is valid whenever $(p, q) \in \partial^{r} u(x, t)$, where $\partial^{r} u(x, t)$ is the set of $(p, q) \in X^{*} \times \mathbb{R}$ such that

$$
\forall(w, r) \in X \times \mathbb{R} \quad\langle p, w\rangle+q r \leq \limsup _{s \rightarrow 0_{+}} s^{-1}(u(x+s w, t+s r)-u(x, t)) .
$$

This set is larger than $\partial u(x, t)$ since the radial (or Dini) upper derivative in the right hand side is larger than the lower derivative.

Similarly, the second part of the proof shows that the inequality $q+H(p) \leq 0$ is valid whenever $(-p,-q) \in$ $\partial^{r}(-u)(x, t)$. Moreover, when $h$ is u.s.c., it suffices that for each $(w, r) \in X \times \mathbb{R}$ one has

$$
\langle p, w\rangle+q r \geq \liminf _{(s, z) \rightarrow\left(0_{+}, w\right)} s^{-1}(u(x+s z, t+r s)-u(x, t)) .
$$

Corollary 6.6. Suppose $H$ is a $w^{*}$-lsc proper convex function and $h=H^{*}$. Then $u$ given by (28) is a Hadamard lower solution and a Hadamard subsolution. If moreover one of the following conditions holds, then $u$ is a subdifferential solution:

(a) for every $(x, t) \in X \times \mathbb{P}$, the inf-convolution in the definition of $u$ is exact at $(x, t)$;

(b) $X$ is reflexive, condition $(C)$ holds and $g$ is weakly l.s.c.;

(c) $X$ is finite dimensional and condition $(C)$ holds;

(d) $g$ is convex.

Proof. The assertions are consequence of the previous results, taking into account the inequality $h^{*}=$ $H^{* *}=H$ and the fact that the Fréchet subdifferential coincides with the Hadamard subdifferential when $X$ is finite dimensional.

Corollary 6.7. Suppose $g$ is convex and $h=H^{*}$. Then $u$ is convex and for any $(x, t) \in X \times \mathbb{P}$, for any $(p, q) \in \partial u(x, t)$ one has $q+H(p) \geq 0$. If moreover $H$ is a l.s.c. proper convex function on $X^{*}$ then for any $(x, t) \in X \times \mathbb{P}$ and for any $(p, q) \in \partial u(x, t)$ one has $q+H(p)=0$.

\section{The LaX-Oleinik Formulas in the QuASICONVEX CASE}

In this section, we closely follow [127], [128] and [18]- [23]; however our assumptions and conclusions are slightly more general. Here $g$ is not assumed to be convex or quasiconvex and $H$ is not necessarily sublinear in its first variable. Moreover $g$ and $H$ may take the value $-\infty$. Again, we introduce some versatility in using a function $\ell$ as a substitute to a conjugate of $H$. We set $\ell_{t}:=\ell\left(t^{-1}\right.$.) and we use the sublevel convolution operation given for two functions $f, g$ by

$$
(f \diamond g)(x)=\inf \{f(u) \vee g(v): u, v \in X, u+v=x\} \quad f, g \in \overline{\mathbb{R}}^{X}, \quad x \in X .
$$

We observe that for any $r, s \in \mathbb{P}$ and for any $x \in X$ one has $\ell_{r+s}(x)=\ell_{r}\left(r(r+s)^{-1} x\right)=\ell_{s}\left(s(r+s)^{-1} x\right)$ and $r(r+s)^{-1} x+s(r+s)^{-1} x=x$, so that $\ell_{r} \diamond \ell_{s} \leq \ell_{r+s}$. If $\ell$ is quasiconvex equality holds, since for any $u, v, x \in X$ with $u+v=x$ one has

$$
\ell_{r+s}(x)=\ell\left(\frac{r}{r+s} \frac{x}{r}+\frac{s}{r+s} \frac{x}{s}\right) \leq \ell\left(\frac{x}{r}\right) \vee \ell\left(\frac{x}{s}\right)=\ell_{r}(x) \vee \ell_{s}(x)
$$

Thus, the semigroup property $\ell_{r} \diamond \ell_{s}=\ell_{r+s}$ for $r, s \in \mathbb{P}$ is satisfied if $\ell$ is quasiconvex, in particular if $\ell:=H^{b}=$ $H^{\sharp}$ (see [128] Lemma 19). Note that assuming the property $\ell=H^{\sharp}\left(=H^{\mathrm{b}}\right)$ ensures that $\ell^{b} \leq H$ but does not imply that $\ell^{\sharp} \leq H$, although this occurs when $H$ is lower right regular (with equality when moreover $H$ is l.s.c. and sublinear in its first variable; see Propositions 2.13, 2.16). 
In this section we define $u:=u_{\sharp}:=u_{b}$ by

$$
u(\cdot, t)=g \triangleright \ell_{t},
$$

The relation $\ell_{r} \diamond \ell_{s} \leq \ell_{t}$ for any $r, s \in \mathbb{P}, t=r+s$ yields

$$
u(\cdot, t) \geq\left(g \triangleright \ell_{t-s}\right) \triangleright \ell_{s}=u(\cdot, t-s) \triangleright \ell_{s},
$$

with equality when $\ell$ is quasiconvex. We also observe that for any functions $f, g$ on $X$ one has $[f \leq r]+[g \leq$ $r] \subset[f \diamond g \leq r]$ and $[f<r]+[g<r]=[f \diamond g<r]$, so that, with the conventions of section 5 ,

$$
\begin{aligned}
& (f \diamond g)^{\sharp} \geq f^{\sharp}+g^{\sharp}, \\
& (f \diamond g)^{b}=f^{b}+g^{b},
\end{aligned}
$$

and, when $\ell^{b} \leq H$ and $g^{b} \leq G$ we have

$$
u(\cdot, t) \geq u(\cdot, t)^{b b}=\left(g^{b}+t \ell^{b}\right)^{b} \geq(G+t H)^{b}=v_{b}(\cdot, t) .
$$

Since for a quasiconvex function $f$ the function $f^{\text {bb }}$ (resp. $f^{\sharp \sharp}$ ) is the l.s.c. hull of $f$ by Proposition 2.8, and since $u(\cdot, t)$ is quasiconvex when $g$ and $\ell$ are quasiconvex, we get parts (a) and (b) of the following result. In part (d) we use the fact that $(f \diamond g)^{\sharp}=f^{\sharp}+g^{\sharp}$ when the sublevel convolution $f \diamond g$ is exact, which means that the infimum in the definition of $f \diamond g$ is attained.

Proposition 7.1. (a) Suppose $H=\ell^{b}>-\infty, G=g^{b}$ with $g, \ell$ quasiconvex. Then, for every $t \in \mathbb{P}$, the function $v_{b}(\cdot, t)=(G+t H)^{b}$ is the l.s.c. hull of $u(\cdot, t)$.

(b) If moreover, for some $(x, t) \in X \times \mathbb{P}$ the function $u(\cdot, t)$ is l.s.c. at $x$, then $u(x, t)=v_{b}(x, t)$.

(c) Suppose $-\infty<H \leq \ell^{\sharp}, G \leq g^{\sharp}$ with $g, \ell$ quasiconvex. Then, for every $t \in \mathbb{P}$, the function $v_{\sharp}(\cdot, t):=$ $(G+t H)^{\sharp}$ is greater than or equal to the l.s.c. hull of $u(\cdot, t)$.

(d) If $-\infty<H=\ell^{\sharp}, G=g^{\sharp}$ with $g, \ell$ quasiconvex, if for some $t \in \mathbb{P}$ the sublevel convolution $\ell_{t} \diamond g$ is exact, and if the function $u(\cdot, t)$ is l.s.c. at $x$, then $u(x, t)=v_{\sharp}(x, t)$.

Proof. (c) When $G \leq g^{\sharp}$ and $H \leq \ell^{\sharp}$, since $\ell_{t}^{\sharp}=t \ell^{\sharp}$ for every $t \in \mathbb{P}$, we have

$$
v_{\sharp}(\cdot, t):=(G+t H)^{\sharp} \geq\left(g^{\sharp}+t \ell^{\sharp}\right)^{\sharp} \geq\left(g \searrow \ell_{t}\right)^{\sharp \sharp}:=u(\cdot, t)^{\sharp \sharp} .
$$

(d) When $u(\cdot, t)$ is l.s.c. at $x$, we obtain $u(x, t)=v_{\sharp}(x, t)$. Moreover, if the sublevel convolution $g \triangleright \ell_{t}$ is exact at $x \in X$, we have $\left(g \triangleright \ell_{t}\right)^{\sharp}=g^{\sharp}+\ell^{\sharp}$, so that the preceding inequalities are equalities when $H=\ell^{\sharp}, G=g^{\sharp}$.

The following example presents a situation in which $u$ coincides with the Lax formula of the convex case. A sufficient condition in order that such a coincidence occurs will be given in section 9 .

Example 12. Let $H$ be given by $H(p, q)=c\|p\|$, with $c>0$, so that its quasiconvex conjugate $\ell:=H^{\sharp}=H^{b}$ is the valley function given by $\ell(x)=-\infty$ for $x \in \bar{B}(0, c), \ell(x)=+\infty$ for $x \in X \backslash \bar{B}(0, c)$. Then, formula (35) gives $u(x, t)=\inf \{g(x-y): y \in \bar{B}(0, c t)\}$, as with the convex Lax formula.

Our first result is similar to Theorem 6.5; the arguments of its proof are inspired by [128] and [94].

Proposition 7.2. (a) Suppose $\ell^{\sharp} \geq H$ and $\ell$ is quasiconvex. Then $u$, as defined in (35), is a normal lower solution to (1), hence a Hadamard lower solution to (1).

(b) If $\ell^{b} \geq H$ and $\ell$ is quasiconvex, $u$ as defined in (35), is a normal subsolution and a subsolution to (1). In particular, these conclusions hold when $\ell=H^{b}, H$ is l.s.c., is a proper (or identically $-\infty$ ) sublinear function of its first variable and is nondecreasing in its second variable.

Proof. (a) Let us prove that $u$ is a normal lower solution when $\ell^{\sharp} \geq H$. Let $(x, t) \in X \times \mathbb{P}, r:=u(x, t)$, and let $(p, q) \in \partial^{\nu} u(x, t)$. Since $\ell$ is quasiconvex, for any $s>0$ and $w \in[\ell \leq r]$, we have

$$
u(x+s w, t+s)=\left(u_{t} \triangleright \ell_{s}\right)(x+s w) \leq u(x, t) \vee \ell_{s}(s w)=u(x, t),
$$


hence $(w, 1) \in T^{r}([u \leq r],(x, t))$, so that $p . w+q \leq 0$. Taking the supremum over $w \in[\ell \leq r]$, and using the assumption that $\ell^{\sharp} \geq H$ (or a fortiori that $\ell^{b} \geq H$ ), we get $H(p, r)+q \leq 0$.

(b) Let $(x, t) \in X \times \mathbb{P}$ and let $(p, q) \in-\partial^{\nu}(-u)(x, t)$. For any $w \in[\ell<r]$, with $r:=u(x, t)$, and any $\left.s \in\right] 0, t[$ we have $u(\cdot, t)=u(\cdot, t-s) \triangleright \ell_{s}$, hence

$$
r:=u(x, t) \leq u(x-s w, t-s) \vee \ell_{s}(s w) .
$$

Thus $u(x, t) \leq u(x-s w, t-s)$ for $s \in[0, t[$. It follows that

$$
(-p) \cdot(-w)+(-q)(-1) \leq 0 .
$$

Therefore, taking the supremum over $w \in[\ell<r]$, and using our assumption that $\ell^{b} \geq H$, we get $H(p, r)+q \leq 0$.

Our next result presents some analogy with Theorem 6.3.

Theorem 7.3. Suppose that for every $(p, r) \in X^{*} \times \mathbb{R}$ one has $\liminf _{s \rightarrow r_{+}} \ell^{b}(p, s) \leq H(p, r)$. If for any $(x, t) \in X \times \mathbb{P}$ with $r:=u(x, t)$ finite the function $k: w \mapsto g(x-w) \vee \ell\left(t^{-1} w\right)$ is quasicoercive, then $u$ is a Fréchet supersolution.

Proof. Let $(x, t) \in X \times \mathbb{P}$ with $r:=u(x, t)$ finite and let $(p, q) \in \partial^{-} u(x, t)$. Let $\left(r_{n}\right) \rightarrow r_{+}$be such that $\lim \ell^{b}\left(p, r_{n}\right) \leq H(p, r)$ and let $\left(s_{n}\right) \rightarrow 0_{+}$be such that $\ell^{b}\left(p, r_{n}\right)<H(p, r)+s_{n}^{2}$. By assumption, there exists a bounded sequence $\left(z_{n}\right)$ of $X$ such that $k\left(z_{n}\right)<\min \left\{r_{n}, r+s_{n}^{2}\right\}$ where $k(w)=g(x-w) \vee \ell\left(t^{-1} w\right)$ for $w \in X$. Then, we have

$$
\begin{aligned}
u\left(x-s_{n} t^{-1} z_{n}, t-s_{n}\right) & =\left(g \triangleright \ell_{t-s_{n}}\right)\left(x-s_{n} t^{-1} z_{n}\right) \\
& \leq g\left(x-z_{n}\right) \vee \ell\left(\left(t-s_{n}\right)^{-1}\left(z_{n}-s_{n} t^{-1} z_{n}\right)\right) \\
& \leq g\left(x-z_{n}\right) \vee \ell\left(t^{-1} z_{n}\right)=k\left(z_{n}\right) \\
& \leq u(x, t)+s_{n}^{2}
\end{aligned}
$$

Since $\left(z_{n}\right)$ is bounded, by definition of $\partial^{-} u(x, t)$, we can find a sequence $\left(\varepsilon_{n}\right) \rightarrow 0_{+}$such that

$$
-p . s_{n} t^{-1} z_{n}-s_{n} q-\varepsilon_{n} s_{n} \leq u\left(x-s_{n} t^{-1} z_{n}, t-s_{n}\right)-u(x, t) \leq s_{n}^{2} .
$$

Therefore, since $\ell\left(t^{-1} z_{n}\right) \leq k\left(z_{n}\right)<r_{n}$,

$$
\ell^{b}\left(p, r_{n}\right) \geq p \cdot t^{-1} z_{n} \geq-s_{n}-\varepsilon_{n}-q
$$

and, by our choices of $\left(r_{n}\right),\left(s_{n}\right)$, we obtain

$$
q+H(p, r) \geq q+\lim _{n} \ell^{b}\left(p, r_{n}\right) \geq 0
$$

Remark. Instead of assuming that for every $(p, r) \in X^{*} \times \mathbb{R}$ one has $\liminf _{s \rightarrow r_{+}} \ell^{b}(p, s) \leq H(p, r)$ one can suppose that for every $(p, r) \in X^{*} \times \mathbb{R}$ one has $e-\lim \sup _{n} \ell^{b}\left(p, r_{n}\right) \leq H(p, r)$ for some sequence $\left(r_{n}\right) \rightarrow r_{+}$in the sense defined in the next section.

Theorem 7.4. Let $u$ be defined by (35) for some arbitrary function $\ell$ on $X$. When the sublevel convolution is exact and $H \geq \ell^{\sharp}$, $u$ is a normal supersolution to (1). If moreover $\ell$ is quasiconvex and $H=\ell^{\sharp}$, then $u$ is such that $q+H(p, u(x, t))=0$ for each $(x, t) \in X \times \mathbb{P}$ and each $(p, q) \in \partial^{\nu} u(x, t)$. 
Proof. Given $(x, t) \in X \times \mathbb{P}$ and $(p, q) \in \partial^{\nu} u(x, t)$, let $z \in X$ be such that $r:=u(x, t)=\ell_{t}(z) \vee g(x-z)$. For any $s \in] 0, t\left[\right.$, setting $z(s):=t^{-1}(t-s) z$ one has $\ell_{t-s}(z(s))=\ell_{t}(z) \leq r$ hence

$$
u(x+z(s)-z, t-s) \leq g(x-z) \vee \ell_{t-s}(z(s)) \leq r .
$$

Since $s^{-1}(z(s)-z)=-t^{-1} z$, it follows that $\left(-t^{-1} z,-1\right)$ is tangent to the sublevel set $[u \leq r]$, hence

$$
p \cdot\left(-t^{-1} z\right)+q(-1) \leq 0 .
$$

Then, when $H \geq \ell^{\sharp}$, since $t^{-1} z \in[\ell \leq r]$, one gets

$$
H(p, r) \geq \ell^{\sharp}(p, r) \geq p .\left(t^{-1} z\right) \geq-q .
$$

Thus $u$ is a normal supersolution.

When $\ell$ is quasiconvex and $H=\ell^{\sharp}$, then, by Proposition 7.2, $u$ is also a normal lower solution, so that $q+H(p, r) \leq 0$.

Corollary 7.5. Suppose $X$ is reflexive. Under one of the following assumptions, the function u defined by (35) is a normal supersolution to (1):

(a) $\ell:=H^{\sharp}, H^{\sharp \sharp}$ is finite, $H^{\sharp \sharp} \leq H$, and $g$ is weakly l.s.c.;

(b) $H \geq \ell^{\sharp}, g$ and $\ell$ are weakly l.s.c. and $g$ is quasicoercive.

If moreover $H=\ell^{\sharp}$ (in particular if $\ell=H^{\sharp}$ and $H$ is sublinear, l.s.c. in its first variable and nondecreasing and right epicontinuous in its second variable) then $u$ is a normal subdifferential solution to (1).

Proof. (a) When $\ell^{\sharp}=H^{\sharp \sharp}$ is finite, the function $\ell$ is coercive (as any sublevel set of $\ell$ is weakly bounded, hence bounded) and weakly l.s.c. If moreover $g$ is weakly l.s.c., the sublevel convolution is exact.

(b) Again, the function $w \mapsto g(w) \vee \ell\left(t^{-1}(x-w)\right)$ is quasicoercive and weakly l.s.c. and so it attains its infimum.

The criteria ensuring $H=H^{\sharp \sharp}$ has been given in Proposition 2.16.

\section{INITIAL CONDITIONS}

It is easy to find solutions to equation (1) alone, even for a badly behaved function $H$. For instance, whenever $H$ is finite at some point $a \in X^{*}$ one can take $u$ given by $u(x, t):=a . x-t H(a)+b$, with $b \in \mathbb{R}$ arbitrary. However the fitting with the initial data is a severe difficulty. If one is willing to accept mild assumptions, one cannot expect that the initial conditions are satisfied in a simple naive sense. The first contributions in this respect are due to Barles and Perthame [17]; cf [19] too. These contributions were not known to the authors when they wrote [128], [94] which were inspired by the developments of variational convergence they were aware of. Let us give a short account of the basic definitions of epi-convergence (see the monographs [6], [8], [29], [45], [113] for instance for much more).

Given a family $\left(f_{t}\right)_{t>0}$ of functions on $X$ parametrized by $\left.\mathbb{P}:=\right] 0, \infty$, its strong epi-limit inferior and its (bounded) weak epi-limit inferior and its are given respectively by

$$
\begin{aligned}
\left(e-\liminf _{t \rightarrow 0_{+}} f_{t}\right)(x) & =\sup _{W \in \mathcal{N}(x)} \liminf _{t \rightarrow 0_{+}} \inf _{w \in W} f_{t}(w)=\liminf _{(w, t) \rightarrow\left(x, 0_{+}\right)} f_{t}(w) \\
\left(e_{w}-\liminf _{t \rightarrow 0_{+}} f_{t}\right)(x) & =\inf _{r>0} \sup _{W \in \mathcal{W}(x)} \liminf _{t \rightarrow 0_{+}} \inf _{w \in W \cap r B} f_{t}(w)=\text { weak } \liminf _{(w, t) \rightarrow\left(x, 0_{+}\right)} f_{t}(w),
\end{aligned}
$$


where $\mathcal{N}(x)$ (resp. $\mathcal{W}(x)$ ) denotes the family of strong (resp. weak) neighborhoods of $x$ and $r B$ is the closed ball with center 0 and radius $r$. Its epi-limit superior is given by

$$
\left(e-\limsup _{t \rightarrow 0_{+}} f_{t}\right)(x):=\sup _{W \in \mathcal{N}(x)} \limsup _{t \rightarrow 0_{+}} \inf _{w \in W} f_{t}(w) .
$$

The family $\left(f_{t}\right)_{t>0}$ is said to Mosco epi-converge to a function $g$ on $X$ if $e_{w}-\liminf _{t \rightarrow 0_{+}} f_{t}=g=e-$ $\lim \sup _{t \rightarrow 0_{+}} f_{t}$; if the weak epi-limit inferior is replaced by the strong epi-limit inferior in this definition, one speaks of epi-convergence. This notion can be given a simple interpretation in terms of set-convergence of epigraphs and is of great importance when dealing with duality questions. It is related to level-convergence, an adapted concept when dealing with quasiconvex functions ( [26], [124]).

Proposition 8.1. Suppose $v$ is given by the Hopf formula $(16): v(x, t):=(G+t F)^{*}(x)$ where $F, G: X^{*} \rightarrow$ $\mathbb{R} \cup\{\infty\}$ are given functions satisfying condition (15)

(a) One has $v_{0}:=v(\cdot, 0) \leq e_{w}-\liminf _{t \rightarrow 0_{+}} v(\cdot, t)$.

(b) One has $v_{0}:=v(\cdot, 0) \leq G^{*}$; if $G^{*} \leq g$, in particular if $G=g^{*}$, one has $v_{0} \leq g^{* *}$.

(c) If $\operatorname{dom} F^{*} \neq \emptyset$ i.e. if $F$ is bounded below by a weak continuous affine function $\langle\cdot, a\rangle-b$, then $v(\cdot, t)$ Mosco epi-converges to $v_{0}: v(\cdot, 0)=e-\lim _{t \rightarrow 0_{+}} v(\cdot, t)$.

(d) If $F$ is bounded below, then $v(\cdot, t)$ pointwise converges to $v_{0}=v(\cdot, 0)$ as $t \rightarrow 0$.

(e) If $\operatorname{dom} G \subset \operatorname{dom} F$, then $v_{0}=G^{*}$; if moreover $G=g^{*}$, one has $v_{0}=g^{* *}$. In particular, if $G^{*}=g$ and $\operatorname{dom} G \subset \operatorname{dom} F$ one has $v_{0}=g$.

(f) If $\operatorname{dom} F^{*} \neq \emptyset$, and if $G^{*}$ is u.s.c. at $x$ (resp. $g$ is u.s.c. at $x$ and $G^{*} \leq g$ ), then one has

$$
\limsup _{(w, t) \rightarrow\left(x, 0_{+}\right)} v(w, t) \leq G^{*}(x) \quad(\text { resp. } g(x)) .
$$

(g) If $\operatorname{dom} G \subset \operatorname{dom} F$, if $\operatorname{dom} F^{*} \neq \emptyset$, and if $G^{*}$ is u.s.c. at $x$ (resp. $g$ is u.s.c. at $x$ and $G^{*}=g$ ), then the restriction of $v$ to $X \times \mathbb{R}_{+}$is continuous at $(x, 0)$ and $v(x, 0)=G^{*}(x)$ (resp. $g(x)$ ).

Proof. (a) If $\operatorname{dom} F \cap \operatorname{dom} G$ is empty, $v_{0}=-\infty^{X}$, the constant function with value $-\infty$, and the inequality is obvious. If $\operatorname{dom} F \cap \operatorname{dom} G$ is nonempty, the definition of $v$ ensures that $v(x, t) \geq p \cdot x-G(p)-t F(p)$ for any $(x, t) \in X \times \mathbb{P}$ and any $p \in \operatorname{dom} F \cap \operatorname{dom} G$, so that

$$
\liminf _{(z, t) \rightarrow\left(x, 0_{+}\right)} v(z, t) \geq \sup \{p \cdot x-G(p): p \in \operatorname{dom} F \cap \operatorname{dom} G\}=\left(G+\iota_{\operatorname{dom} F}\right)^{*}(x):=v_{0}(x) .
$$

Another argument has been given in Lemma 4.1.

(b) Since $G+\iota_{\operatorname{dom} F} \geq G$ we get $v(\cdot, 0):=\left(G+\iota_{\operatorname{dom} F}\right)^{*} \leq G^{*}$, hence $v_{0} \leq g$ when $G^{*} \leq g$.

(c) When $F \geq\langle\cdot, a\rangle-b$, for every $t>0$ we have $t F \geq \iota_{\operatorname{dom} F}+\langle\cdot, t a\rangle-t b$, hence

$$
v(w, t) \leq\left(G+\iota_{\operatorname{dom} F}\right)^{*}(w-t a)+t b \quad \forall w \in X .
$$

Thus, for any $x \in X$ and any sequence $\left(t_{n}\right) \rightarrow 0_{+}$, taking $\left(w_{n}\right):=\left(x+t_{n} a\right)$ which converges to $x$, we get

$$
\left(e-\lim \sup v\left(\cdot, t_{n}\right)\right)(x) \leq \limsup _{n} v\left(w_{n}, t_{n}\right) \leq \lim _{n}\left(\left(G+\iota_{\operatorname{dom} F}\right)^{*}(x)+t_{n} b\right)=v(x, 0) .
$$

In fact, the estimates $v(\cdot, 0) \leq v(\cdot, t)$ and $(36)$ show that $v(\cdot, t)$ boundedly converges to $v(\cdot, 0)$ in the sense of [109].

(d) When $F$ is bounded below, we can take $a=0$ in what precedes, so that $v(x, t) \leq\left(G+\iota_{\operatorname{dom} F}\right)^{*}(x)+t b=$ $v(x, 0)+t b$. Taking (a) into account we get that $v(x, t) \rightarrow v(x, 0)$ as $t \rightarrow 0_{+}$.

(e) If $\operatorname{dom} G \subset \operatorname{dom} F$ we have $G+\iota_{\operatorname{dom} F}=G$, hence, when $G^{*}=g$,

$$
v(\cdot, 0)=\left(G+\iota_{\operatorname{dom} F}\right)^{*}=G^{*}=g .
$$


(f) If $\operatorname{dom} F^{*} \neq \emptyset$ and if $G^{*}$ is u.s.c. at $x$, from (36) we obtain

$$
\limsup _{(w, t) \rightarrow\left(x, 0_{+}\right)} v(w, t) \leq \limsup _{(w, t) \rightarrow\left(x, 0_{+}\right)}\left(G+\iota_{\operatorname{dom} F}\right)^{*}(w-t a)+t b \leq \limsup _{(w, t) \rightarrow\left(x, 0_{+}\right)}\left(G^{*}(w-t a)+t b\right) \leq G^{*}(x) .
$$

When $G^{*} \leq g$ and $g$ is u.s.c. at $x$, one can replace $G^{*}(x)$ by $g(x)$ in the last inequality.

(g) It is a consequence of (e) and (f).

In the following proposition which deals with the Lax solution, we use the asymptotic function of $h$ and the incident asymptotic function of $h$ given respectively by

$$
\begin{aligned}
& h_{\infty}(z):=\left(e_{w}-\liminf _{t \rightarrow \infty} h_{t}\right)(z)=\text { weak }-\liminf _{(w, t) \rightarrow(z, \infty)} t^{-1} h(t w), \\
& h_{\infty}^{i}(z):=e-\limsup _{t \rightarrow \infty} t^{-1} h(t \cdot)(z) \text { for } z \in X,
\end{aligned}
$$

where $h_{t}(w):=t^{-1} h(t w)$ and where the convergence on $X$ is the bounded weak convergence and the strong convergence respectively. Thus $h_{\infty}(z)$ is the infimum of the limits of $t_{i}^{-1} h\left(t_{i} z_{i}\right)$ over the family of nets $\left(t_{i}, z_{i}\right)_{i \in I}$ such that $\left(t_{i}\right) \rightarrow 0_{+},\left(z_{i}\right)_{i \in I} \rightarrow z$ weakly, $\left(z_{i}\right)_{i \in I}$ being bounded. Let us note that when $h$ is a l.s.c. proper convex function one has $h_{\infty}^{i}=h_{\infty}$. These functions can be defined with the help of the asymptotic cones of the epigraph of $h$. We say that $g$ is calm at $x \in X$ with rate $c \in \mathbb{R}_{+}$if there exists a neighborhood $W$ of $x$ such that

$$
\forall w \in W \quad g(x-w) \geq g(x)-c\|w\| .
$$

Assertion (e) of the next proposition is inspired by [4] Theorem 5.2. It uses the following well-known fact; we prove it for the sake of completeness.

Lemma 8.2. Let $g: X \rightarrow \mathbb{R} \cup\{+\infty\}$ be l.s.c. at $x \in X$, such that $\liminf _{\|x\| \rightarrow \infty} g(x) /\|x\|>-\infty$ and bounded below on each ball. Let $g_{n}$ be the Baire regularized function of g given by $g_{n}(x):=\inf _{w \in X}(g(w)+n\|x-w\|)$. Then $\left(g_{n}\right) \rightarrow g$ pointwise.

Proof. By assumption, there exist $b \in \mathbb{R}, c \in \mathbb{R}_{+}$such that $g(w) \geq b-c\|w\|$. Let $a<g(x)$. Since $g$ is l.s.c. at $x$ we can find $r>0$ such that $g(w)>a$ for $w \in B(x, r)$. For $w \in X \backslash B(x, r)$ and for $n$ large enough, we have

$$
g(w)+n\|x-w\| \geq b-c\|w\|+n\|x-w\| \geq b-c\|x\|+(n-c) r>a,
$$

hence $g_{n}(x)>a$. Since $g_{n}(x) \leq g(x)$ for each $n$, we get that $\left(g_{n}(x)\right) \rightarrow g(x)$.

In the next statement we take $F:=h^{*}, G:=g^{*}, v(x, t):=(G+t F)^{*}(x), v_{0}(x):=\left(G+\iota_{\text {dom } F}\right)^{*}(x)$, and we say that $g$ is globally calm at $x$ with rate $c$ if for every $w \in X$ we have $g(x-w) \geq g(x)-c\|w\|$.

Proposition 8.3. Suppose $u$ is given by formula (28): $u(x, t):=\left(g \square h_{t}\right)(x)$ and (29) holds.

(a) One has $v_{0} \leq e_{w}-\liminf _{t \rightarrow 0_{+}} u(\cdot, t) \leq g \square h_{\infty} \leq g$ and $e-\limsup _{t \rightarrow 0_{+}} u(\cdot, t) \leq g \square h_{\infty}^{i} \leq g$. If $\operatorname{dom} g^{*} \subset \operatorname{dom} F$ one has $g^{* *} \leq e_{w}-\liminf _{t \rightarrow 0_{+}} u(\cdot, t)$.

(b) If $h(0)<\infty$, one has $e-\lim \sup _{t \rightarrow 0_{+}} u(\cdot, t) \leq \lim \sup _{t \rightarrow 0_{+}} u(\cdot, t) \leq g$; in particular this holds when $h \leq H^{*}$ and $H$ is bounded below.

(c) Suppose condition $(C)$ : $\liminf _{\|w\| \rightarrow \infty} h(w) /\|w\|>-\liminf _{\|w\| \rightarrow \infty} g(w) /\|w\|$. Suppose also that $X$ is reflexive and that the restriction of $g$ to any bounded set is weakly l.s.c. Then $e_{w}-\liminf _{t \rightarrow 0_{+}} u(\cdot, t)=g \square h_{\infty}$ and if $h_{\infty}^{i}=h_{\infty}$ then $u(\cdot, t)$ Mosco converges to $u_{0}:=g \square h_{\infty}$.

(d) Suppose $X$ is finite dimensional, $g$ is l.s.c., and

$$
g_{\infty}(-z)>-h_{\infty}(z) \quad \text { for each } z \in X \backslash\{0\} .
$$

Then $e-\liminf _{t \rightarrow 0} u(\cdot, t)=g \square h_{\infty}$. If moreover $h_{\infty}=h_{\infty}^{i}$ then $u(\cdot, t)$ epi-converges to $g \square h_{\infty}$ as $t \rightarrow 0_{+}$. 
(e) If $g$ and $h$ are bounded below on each ball and are such that $h(0)<\infty$,

$$
\liminf _{\|w\| \rightarrow \infty} g(w) /\|w\|>-\infty, \quad \liminf _{\|w\| \rightarrow \infty} h(w) /\|w\|=+\infty,
$$

and if $g$ is l.s.c. at $x$, then $u(x, t) \rightarrow g(x)$ as $t \rightarrow 0_{+}$.

(f) If $g$ is l.s.c. at $x$, if $h$ is bounded below and if there exist some nondecreasing functions $\varphi, \psi: \mathbb{R}_{+} \rightarrow \mathbb{R}$ such that $g \geq-\varphi(\|\cdot\|), h \geq \psi(\|\cdot\|)$ and such that for any $c \in \mathbb{R}_{+}, \delta>0 \lim _{t \backslash 0} \inf \left\{t \psi\left(t^{-1} r\right)-\varphi(r+c): r \geq \delta\right\}=+\infty$, then $u(x, t) \rightarrow g(x)$ as $t \rightarrow 0_{+}$.

(g) Suppose that $g$ is globally calm with rate $c>0$ at some $x \in X$ and that $h^{*}$ is bounded above on $B(0, c)$. Then $g(x)=\left(g \square h_{\infty}\right)(x)=e-\liminf _{t \rightarrow 0} u(\cdot, t)(x)$. If moreover $g$ is Lipschitzian with rate $c$, then $u(\cdot, t)$ epiconverges to $g$ as $t \rightarrow 0_{+}$.

Let us note that in assertion (f) we can take $\varphi(r)=a r^{s}-m, \psi(r)=b r^{s}-n$ with $s>1, a, b>0, m, n \in \mathbb{R}$.

Proof. (a) We have $v(x, t)=\left(g^{*}+t h^{*}\right)^{*}(x)=\left(g \square h_{t}\right)^{* *}(x) \leq u(x, t)$ so that assertion (a) of Proposition 8.1 yields

$$
v_{0} \leq e_{w}-\liminf _{t \rightarrow 0_{+}} v(\cdot, t) \leq e_{w}-\liminf _{t \rightarrow 0_{+}} u(\cdot, t) .
$$

Given $(x, y) \in X \times X$ one can find a bounded net $\left(\left(y_{i}, t_{i}\right)\right)_{i \in I} \rightarrow\left(y, 0_{+}\right)$in the weak topology such that $h_{\infty}(y)=\lim _{i \in I} t_{i} h\left(y_{i} / t_{i}\right)$. Then one has

$$
\begin{aligned}
e_{w}-\liminf _{t \rightarrow 0_{+}} u(\cdot, t)(x) & \leq \liminf _{i \in I} u\left(x-y+y_{i}, t_{i}\right) \\
& \leq \liminf _{i \in I}\left(g(x-y)+t_{i} h\left(\frac{y_{i}}{t_{i}}\right)\right)=g(x-y)+h_{\infty}(y) .
\end{aligned}
$$

Taking the infimum on $y \in X$, one gets $e_{w}-\liminf _{t \rightarrow 0_{+}} u(\cdot, t) \leq g \square h_{\infty}$. Since for any $a \in \operatorname{dom} h$ one has $h_{\infty}(0) \leq h_{\infty}^{i}(0) \leq \lim _{t \rightarrow 0_{+}} t h\left(t^{-1} t a\right) \leq 0$, one gets $g \square h_{\infty} \leq g \square h_{\infty}^{i} \leq g$. Given $x \in X$ and $r>\left(g \square h_{\infty}^{i}\right)(x)$, one can pick $y \in X$ such that $g(x-y)+h_{\infty}^{i}(y)<r$. Then, as $h_{\infty}^{i}=e-\lim \sup _{t \rightarrow 0_{+}} h_{t}$, for any sequence $\left(t_{n}\right) \rightarrow 0_{+}$ one can find a sequence $\left(y_{n}\right) \rightarrow y$ such that $\lim \sup _{n} t_{n} h\left(y_{n} / t_{n}\right) \leq h_{\infty}^{i}(y)$ so that, for $x_{n}:=x-y+y_{n}$, and for $n$ large enough, one has

$$
u\left(x_{n}, t_{n}\right) \leq g\left(x_{n}-y_{n}\right)+t_{n} h\left(\frac{y_{n}}{t_{n}}\right)=g(x-y)+t_{n} h\left(\frac{y_{n}}{t_{n}}\right)<r .
$$

Since $\left(x_{n}\right) \rightarrow x$, we get $e-\lim \sup _{t \rightarrow 0_{+}} u(\cdot, t)(x) \leq r$. Hence $e-\limsup _{t \rightarrow 0_{+}} u(\cdot, t) \leq g \square h_{\infty}^{i} \leq g$. When $\operatorname{dom} g^{*} \subset \operatorname{dom} F$, Proposition 8.1 (e) with $F:=h^{*}, G:=g^{*}$, ensures that $v_{0}=g^{* *}$.

(b) The first inequality is a consequence of the definitions; the second one follows from $\left(g \square h_{t}\right)(x) \leq g(x)+$ th(0) for $(x, t) \in X \times \mathbb{P}$.

(c) Let $b>c>-\liminf _{\|x\| \rightarrow \infty} g(x) /\|x\|$, with $b<\liminf _{\|x\| \rightarrow \infty} h(x) /\|x\|$. We can find some $d \in \mathbb{R}_{+}$ such that $h(w) \geq b\|w\|, g(w) \geq-c\|w\|$ for each $w \in X \backslash B(0, d)$. If $e_{w}-\liminf _{t \rightarrow 0_{+}} u(\cdot, t)(x)=+\infty$, then $g \square h_{\infty}=+\infty$ by (a). Let $s \in \mathbb{R}$ be such that $s>e_{w}-\liminf _{t \rightarrow 0_{+}} u(\cdot, t)(x)$. There exist $r>0$ and nets $\left(t_{i}\right)_{i \in I} \rightarrow 0_{+},\left(x_{i}\right)_{i \in I} \rightarrow x$ in the weak topology with $x_{i} \in r B$ for each $i \in I$ such that $s>\liminf _{i \in I} u\left(x_{i}, t_{i}\right)$. Taking a subnet if necessary, we may assume that $s>u\left(x_{i}, t_{i}\right)$ for each $i \in I$ and we may pick some $w_{i} \in X$ such that

$$
s>g\left(x_{i}-w_{i}\right)+t_{i} h\left(w_{i} / t_{i}\right) .
$$

Then, if $\left(w_{i}\right)$ is unbounded, taking a further subnet if necessary, we have

$$
s>-c\left\|x_{i}-w_{i}\right\|+b\left\|w_{i}\right\| \geq(b-c)\left\|w_{i}\right\|-|c| r,
$$

for each $i$, a contradiction. Thus $\left(w_{i}\right)_{i \in I}$ is bounded and taking another subnet if necessary, we may suppose $\left(w_{i}\right)_{i \in I}$ converges to some $w$ in the weak topology. Then, as $g$ is weakly l.s.c. on bounded sets, we deduce from 
(39) that

$$
s \geq \liminf _{i \in I} g\left(x_{i}-w_{i}\right)+h_{\infty}(w) \geq g(x-w)+h_{\infty}(w) \geq\left(g \square h_{\infty}\right)(x) .
$$

In view of (a), the equality $\left(g \square h_{\infty}\right)(x)=e-\liminf _{t \rightarrow 0} u(\cdot, t)(x)$ holds. If moreover $h_{\infty}^{i}=h_{\infty}$, we have $e-\lim \sup _{t \rightarrow 0_{+}} u(\cdot, t) \leq g \square h_{\infty}$ by (a) and we get Mosco convergence.

(d) Suppose on the contrary that $e-\liminf _{t \rightarrow 0} u(\cdot, t)(x)<\left(g \square h_{\infty}\right)(x)$ for some $x \in X$ : there exist $r<$ $\left(g \square h_{\infty}\right)(x)$ and sequences $\left(t_{n}\right) \rightarrow 0_{+},\left(x_{n}\right) \rightarrow x$ such that $\left(u\left(x_{n}, t_{n}\right)\right) \rightarrow r$. The definition of an infimal convolution ensures that we can find a sequence $\left(w_{n}\right)$ such that

$$
g\left(x_{n}-w_{n}\right)+t_{n} h\left(t_{n}^{-1} w_{n}\right) \rightarrow r \quad \text { as } n \rightarrow \infty .
$$

Taking subsequences, we may assume that either $\left(w_{n}\right)$ has a limit $w$ or that $\left(s_{n}\right):=\left(\left\|w_{n}\right\|\right) \rightarrow \infty$ and $\left(z_{n}\right):=$ $\left(s_{n}^{-1} w_{n}\right)$ has a limit $z$ with norm 1 . In the first case we get

$$
r \geq g(x-w)+h_{\infty}(w) \geq\left(g \square h_{\infty}\right)(x)>r,
$$

a contradiction. In the second case, we get

$$
\begin{aligned}
g_{\infty}(-z)+h_{\infty}(z) & \leq \liminf _{n} s_{n}^{-1} g\left(s_{n}\left(s_{n}^{-1} x_{n}-z_{n}\right)\right)+\liminf _{n} s_{n}^{-1} t_{n} h\left(s_{n} t_{n}^{-1} z_{n}\right) \\
& \leq \liminf _{n} s_{n}^{-1}\left(g\left(x_{n}-w_{n}\right)+t_{n} h\left(t_{n}^{-1} w_{n}\right)\right)=0,
\end{aligned}
$$

a contradiction with our assumption. Therefore $e-\liminf _{t \rightarrow 0} u(\cdot, t) \geq g \square h_{\infty}$. The last assertion follows from that and from (a).

(e) By assumption, there exist $d \in \mathbb{R}, c \in \mathbb{R}_{+}$such that $g(\cdot) \geq d-c\|\cdot\|$ and for each $n \in \mathbb{N}$ there exists $m_{n} \in \mathbb{R}$ such that $h(\cdot) \geq n\|\cdot\|-m_{n}$ (this last condition is satisfied if $h \geq H^{*}$ and if $H^{* *}(p) \leq m_{n}$ for each $p \in B(0, n))$. Then

$$
u(x, t)=\inf _{w \in X}\left(g(x-w)+\operatorname{th}\left(\frac{w}{t}\right)\right) \geq \inf _{w \in X}\left(g(x-w)+n\|w\|-t m_{n}\right)=g_{n}(x)-t m_{n} .
$$

hence $\liminf \inf _{t \rightarrow 0} u(x, t) \geq g_{n}(x)$ for each $n \in \mathbb{N}$, and the preceding lemma entails $\liminf _{t \rightarrow 0} u(x, t) \geq g(x)$. When $h(0)<\infty$, assertion (b) gives $\lim _{\sup _{t \rightarrow 0}} u(x, t) \leq g(x)$, so that $u(x, t) \rightarrow g(x)$ as $t \rightarrow 0_{+}$.

(f) Given $\lambda \in \mathbb{R}$ less than $g(x)$ and $\varepsilon>0$ with $\lambda+2 \varepsilon<g(x)$, let $\delta>0$ be such that $g(x-y) \geq \lambda+\varepsilon$ for $y \in B(0, \delta)$. Let $m>0$ be such that $h \geq-m$. Then, if $t \in] 0, \varepsilon / m[$, for each $y \in B(0, \delta)$ we have

$$
g(x-y)+\operatorname{th}\left(t^{-1} y\right) \geq \lambda .
$$

For $r:=\|y\| \geq \delta, c:=\|x\|$, our assumption ensures that, for $t>0$ small enough,

$$
g(x-y)+t h\left(t^{-1} y\right) \geq-\varphi(r+c)+t \psi\left(t^{-1} r\right) \geq \lambda .
$$

Thus $\left(g \square h_{t}\right)(x) \geq \lambda$ for $t>0$ small enough.

(g) Suppose $g$ is calm at $x$ with rate $c$ and that for some $m<\infty$ and each $p \in B(0, c)$ we have $h^{*}(p) \leq m$ (or a fortiori $H(p) \leq m$ and $h^{*} \leq H$ or $H^{* *}(p) \leq m$ and $h \geq H^{*}$ ). Then, for each $w \in X$, we have

$$
h(w) \geq \sup _{p \in B(0, c)}\left(\langle p, w\rangle-h^{*}(p)\right) \geq c\|w\|-m .
$$

Using global calmness of $g$, we get

$$
u(x, t)=\inf _{w \in X}\left(g(x-w)+\operatorname{th}\left(\frac{w}{t}\right)\right) \geq g(x)-t m,
$$


so that $\liminf \inf _{t \rightarrow 0} u(x, t) \geq g(x)$. Moreover, if $\left(x_{i}\right)_{i \in I} \rightarrow x$ in norm and $\left(t_{i}\right)_{i \in I} \rightarrow 0_{+}$, we can pick $w_{i} \in X$ such that

$$
\begin{aligned}
u\left(x_{i}, t_{i}\right)+t_{i} & >g\left(x_{i}-w_{i}\right)+t_{i} h\left(\frac{w_{i}}{t_{i}}\right) \\
& \geq g(x)-c\left\|x_{i}-w_{i}-x\right\|+c\left\|w_{i}\right\|-t_{i} m \\
& \geq g(x)-c\left\|x_{i}-x\right\|-t_{i} m
\end{aligned}
$$

Thus $\liminf i_{i} u\left(x_{i}, t_{i}\right) \geq g(x)$. This proves that $e-\liminf _{t \rightarrow 0} u(\cdot, t)(x) \geq g(x)$ and (a) ensures equality. Now let us observe that our assumptions imply that $\left(g \square h_{\infty}\right)(x)=g(x)$ : since relation $(40)$ implies that $h_{\infty}(w) \geq c\|w\|$ for each $w \in X$, we get

$$
g(w)+h_{\infty}(x-w) \geq g(x)-c\|w-x\|+c\|x-w\|=g(x),
$$

hence $\left(g \square h_{\infty}\right)(x) \geq g(x)$.

If $g$ is Lipschitzian with rate $c$, using (a) and the relation $g \square h_{\infty}=g$ we have just proved, we get $g \geq$ $e-\lim \sup _{t \rightarrow 0_{+}} u(\cdot, t) \geq e-\liminf _{t \rightarrow 0} u(\cdot, t)(x) \geq g$.

Remarks. (a) The relation $h_{\infty}(-z)>-g_{\infty}(z)$ for each $z \in X \backslash\{0\}$ is a consequence of condition (C). In fact for each unit vector $z$ one has $g_{\infty}(z) \geq \liminf _{\|w\| \rightarrow \infty} g(w) /\|w\|, h_{\infty}(z) \geq \liminf _{\|w\| \rightarrow \infty} h(w) /\|w\|$

(b) It is observed in [63] that when $g$ and $h$ are l.s.c. proper convex functions the condition $g \square h_{\infty}=g$ is equivalent to the assumption $\operatorname{cl}\left(\operatorname{dom} g^{*}\right) \subset \operatorname{cl}\left(\operatorname{dom} h^{*}\right)$ (see [73] Théorème 6.8.5).

Combining the results of the preceding two propositions, we get new assertions.

Corollary 8.4. Let $u$ and $v$ be given by $u(x, t):=\left(g \square h_{t}\right)(x), v(x, t):=\left(g^{*}+t\right)^{*}(x)$. Suppose $F=h^{*}, G=g^{*}$, $g=g^{* *}$ (29) holds, and $\operatorname{dom} g^{*} \subset \operatorname{dom} h^{*}$.

(a) Then $u(\cdot, t)$ and $v(\cdot, t)$ Mosco converge to $g$.

(b) If moreover $h(0)<+\infty$, then $u(\cdot, t)$ and $v(\cdot, t)$ pointwise converge to $g$ as $t \rightarrow 0_{+}$.

Proof. (a) Since $v_{0}:=v(\cdot, 0)=g$ by assertion (e) of Proposition 8.1 in which we take $F:=h^{*}, G:=g^{*}$, we get, by (a) of Propositions 8.1, 8.3,

$$
\begin{aligned}
& g \leq e_{w}-\liminf _{t \rightarrow 0_{+}} v(\cdot, t) \leq e_{w}-\liminf _{t \rightarrow 0_{+}} u(\cdot, t) \leq e-\limsup _{t \rightarrow 0_{+}} u(\cdot, t) \leq g \\
& g \leq e_{w}-\liminf _{t \rightarrow 0_{+}} v(\cdot, t) \leq e-\limsup _{t \rightarrow 0_{+}} v(\cdot, t) \leq e-\limsup _{t \rightarrow 0_{+}} u(\cdot, t) \leq g
\end{aligned}
$$

These inequalities ensure that $v(\cdot, t)$ and $u(\cdot, t)$ Mosco converges to $g$.

(b) If moreover $h(0)<+\infty$ Proposition 8.3 (b) ensures that $e-\liminf _{t \rightarrow 0_{+}} u(\cdot, t)=g \square h_{\infty}$. Hence, by (a) we have $g \square h_{\infty}=g$ we obtain by (b) of Proposition 8.3

$$
\begin{aligned}
& g=e_{w}-\liminf _{t \rightarrow 0_{+}} v(\cdot, t) \leq \liminf _{t \rightarrow 0_{+}} v(\cdot, t) \leq \liminf _{t \rightarrow 0_{+}} u(\cdot, t) \leq \limsup _{t \rightarrow 0_{+}} u(\cdot, t) \leq g, \\
& g \leq \liminf _{t \rightarrow 0_{+}} v(\cdot, t) \leq \limsup _{t \rightarrow 0_{+}} v(\cdot, t) \leq \limsup _{t \rightarrow 0_{+}} u(\cdot, t) \leq g
\end{aligned}
$$

Thus $v(\cdot, t)$ and $u(\cdot, t)$ pointwise converges to $g$.

Now let us turn to the formulas of quasiconvex type. Let us start with Hopf type formulas.

The relation $v_{0} \leq e-\liminf _{t \backslash 0} v_{t}$ is a consequence of Lemma 23 .

Now let us consider the Lax-Oleinik formula using the sublevel convolution. We just quote the following result.

Proposition 8.5. ([94, Prop. 12.13], [105]) Suppose $g$ is weakly l.s.c., $H$ is finite and inf $g \geq \inf h$. Let $h_{t}:=(t H)^{b}=(t H)^{\sharp}$. Then the Lax solution $u_{t}:=g \diamond h_{t}$ epiconverges to $g$ as $t \rightarrow 0_{+}$. 


\section{Comparison and uniqueness Results}

The use of the substitutes $F, G, h$ to $H, g^{*}, H^{*}$ respectively in the preceding sections has not much interest if one is unable to compare solutions. Happily, such results have been obtained in the literature (see the comprehensive monograph [13] for instance). They lead to uniqueness results. Uniqueness results are strong and delicate points in the viscosity approach to Hamilton-Jacobi equations ( [13], [16], [39], [40]). Another notable uniqueness result due to Barron and Jensen concerns l.s.c. solutions or unilateral solutions ( [19], see also [16], [13]); however the technique used seems to be limited to the finite dimensional case.

Uniqueness properties can be obtained through comparison results. A classical means is to use the value function of the Bolza problem

$$
u_{B}(x, t):=\inf \left\{j_{x, t}(w): w \in W^{1,1}([0, t], X), w(t)=x\right\}
$$

where $L:=H^{*}$,

$$
j_{x, t}(w):=g(w(0))+\int_{0}^{t} L\left(w^{\prime}(s)\right) d s .
$$

Proposition 9.1. For any function $H$ the value function $u_{B}$ just defined coincides with the Lax solution $u$ given by (28) in which $h:=H^{*}$.

We give the proof for completeness; it suffices to check that the coercivity assumption on $H^{*}$ and the Lipschitz assumption of $g$ in [34, Thm 1.3.1], [51] can be avoided. It can also be seen that the value function can be defined with arcs of class $C^{1}$.

Proof. Given $x, y \in X, t \in \mathbb{P}$, setting $w(s):=y+s t^{-1}(x-y)$ for $s \in[0, t]$, we see that

$$
u_{B}(x, t) \leq j_{x, t}(w)=g(y)+t H^{*}\left(t^{-1}(x-y)\right)
$$

hence, taking the infimum over $y \in X, u_{B}(x, t) \leq u(x, t)$. Conversely, since for every $w \in W^{1,1}([0, t], X)$ satisfying $w(t)=x$, we have, for $y:=w(0)$ and an arbitrary $p \in \operatorname{dom} H$,

$$
\begin{aligned}
j_{x, t}(w) & :=g(y)+\int_{0}^{t} H^{*}\left(w^{\prime}(s)\right) d s \geq g(y)+\int_{0}^{t}\left(p \cdot w^{\prime}(s)-H(p)\right) d s \\
& \geq g(y)+p \cdot(x-y)-t H(p),
\end{aligned}
$$

hence $j_{x, t}(w) \geq g(y)+(t H)^{*}(x-y) \geq u(x, t)$ by taking the supremum over $p$ and then the infimum over $y$. Taking the infimum over $w \in W^{1,1}([0, \bar{t}], X)$, satisfying $w(t)=x$, we get $u_{B}(x, t) \geq u(x, t)$ and equality holds. $\square$

The value function can be used for more general Lagrangians and Hamiltonians; see [13], [24], [35], [46], [58], [78], [121], [123] and their references. In such a case different control problems, such as $L_{\infty}$ control problems, or differential games have to be considered.

The explicit forms of the Hopf and the Lax solutions entail easy comparison results:

$$
\begin{aligned}
g \leq g^{\prime}, h \leq h^{\prime} & \Longrightarrow g \square h_{t} \leq g^{\prime} \square h_{t}^{\prime} & & \forall t \in \mathbb{P}, \\
G \geq G^{\prime}, F \geq F^{\prime} & \Longrightarrow(G+t F)^{*} \leq\left(G^{\prime}+t F^{\prime}\right)^{*} & & \forall t \in \mathbb{P}, \\
& \Longrightarrow(G+t F)^{\sharp} \leq\left(G^{\prime}+t F^{\prime}\right)^{\sharp} & & \forall t \in \mathbb{P}
\end{aligned}
$$

and similar implications for the other Hopf formulas.

We start with a comparison between $v_{\%}$ and $v_{\sharp}$ and their variants which slightly completes [4, Thm 6.10].

Proposition 9.2. Suppose $H$ is nondecreasing in its second variable. Then, for every $t>0$ one has $v_{\%}(\cdot, t):=$ $\left(g^{\%}+t H\right)^{\%} \leq v_{\div}(\cdot, t):=\left(g^{\div}+t H\right)^{\div} \leq v_{\sharp}(\cdot, t):=\left(g^{\sharp}+t H\right)^{\sharp} \leq v_{b}(\cdot, t):=\left(g^{b}+t H\right)^{b}$. If moreover $g=g^{\% \%}$ is bounded below by a continuous affine function, if there exists some $r^{\prime}>r:=v_{\%}(x, t)$ such that for every 
$s \in] r, r^{\prime}$ [ the function $H(\cdot, s)$ is positively homogeneous, and one of the following assumptions holds, then these inequalities are equalities:

(a) for every $s \in] r, r^{\prime}[$ the function $H(\cdot, s)$ is u.s.c.;

(b) for every $s \in] r, r^{\prime}\left[\right.$ the function $H(\cdot, s)$ is sublinear and $\operatorname{dom} H(\cdot, s) \cap \operatorname{dom} g^{*} \neq \varnothing$;

(c) the sublevel set $\left[g \leq r^{\prime}\right]$ is bounded and $H$ is right lower regular.

Proof. Since $g^{\div} \leq g^{\%}$, we have $\left(g^{\%}+t H\right)^{\%}=\left(g^{\%}+t H\right) \div \leq(g \div+t H) \div$ and similarly $\left(g^{\sharp}+t H\right)^{\sharp} \leq\left(g^{b}+t H\right)^{b}$. Let us show that $v_{\%}(x, t) \leq v_{b}(x, t)$ for every $(x, t) \in X \times \mathbb{P}$. Let $q:=v_{b}(x, t)$. For every $s^{\prime}>s>q$ we have $\left(g_{s}^{b}+t H_{s}\right)^{*}(x) \leq 0$, or $g^{b}(p, s)+t H(p, s) \geq p . x$ for every $p \in X^{*}$, hence, since $g^{\div}\left(p, s^{\prime}\right) \geq g^{b}(p, s)-s$ by Lemma 2.2,

$$
g^{\circ}\left(p, s^{\prime}\right)+t H(p, s) \geq p . x-s .
$$

Taking the infimum over $s^{\prime}>s>q$, i.e. the limits as $s, s^{\prime} \rightarrow q_{+}$, we get

$$
g \div(p, q+0)+t H(p, q+0) \geq p \cdot x-q .
$$

Thus $\left(g_{\dot{q}+0}^{\dot{\bar{*}}}+t H_{q+0}\right)^{*}(x) \leq q$ and $v_{\div}(x, t) \leq q$ by Lemma 2.4 .

To prove that $v_{b}(x, t) \leq v_{\%}(x, t)$ under the additional assumptions, we follow [4, Thm 4.6]. Let $r:=v_{\%}(x, t)$; we may suppose $r<+\infty$. When $r \geq \sup g$, for all $p \in X^{*}$ we have $g^{\%}(p, r+0)=g^{*}(p)$, hence, by Lemma 2.4 and the definition of $v_{\%}(x, t)$,

$$
g^{*}(p)+t H_{r+0}(p)-p . x \geq-r .
$$

Since $g$ is bounded below by some continuous affine function, the domain of $g^{*}$ is nonempty. Thus, inequality (43) ensures that $H_{r+0}$ is not identically equal to $-\infty$ and since $H_{r+0}$ is positively homogeneous in the sense we have adopted, we have $H_{r+0}(0)=0$. On the other hand, since $\left[g<r^{\prime}\right]=X$ for every $r^{\prime}>r$, we have $g^{b}(p, r+0)=\iota_{\{0\}}(p)$, hence

$$
\left(g_{r+0}^{b}+t H_{r+0}\right)^{*}(x)=-H_{r+0}(0)=0 .
$$

Thus $v_{b}(x, t) \leq r$ in the case $r \geq \sup g$. When $r<\sup g$, we pick $\left.r^{\prime} \in\right] r, \sup g$ [, so that we can find $a \in X^{*}$ and $b \in \mathbb{R}$ such that $g(x) \geq a . x+b$ for every $x \in\left[g \leq r^{\prime}\right]$. Then, for $p \in X^{*}$ and $\left.\left.s, s^{\prime} \in\right] r, r^{\prime}\right]$ with $s<s^{\prime}$, we have

$$
g^{\%}(p, s) \leq \sup \left\{(p-a) \cdot x-b: x \in\left[g<s^{\prime}\right]\right\}=g^{b}\left(p-a, s^{\prime}\right)-b
$$

Then, the definition of $v_{\%}(x, t)$ yields

$$
g^{b}\left(p-a, s^{\prime}\right)-b+t H(p, s) \geq g^{\%}(p-a, s)+t H(p, s) \geq p . x-r .
$$

Replacing $p$ by $a+\lambda p^{\prime}$ with $\lambda \in \mathbb{P}, p^{\prime} \in X^{*}$ arbitrary and dividing by $\lambda$, we obtain

$$
g^{b}\left(p^{\prime}, s^{\prime}\right)-\lambda^{-1} b+t H\left(\lambda^{-1} a+p^{\prime}, s\right) \geq\left(\lambda^{-1} a+p^{\prime}\right) \cdot x-\lambda^{-1} r,
$$

as $H(\cdot, s)$ is positively homogeneous. When $H(\cdot, s)$ is u.s.c., taking the limit as $\lambda \rightarrow+\infty$, we get

$$
\left.\left.\forall s^{\prime} \in\right] r, r^{\prime}\right], \forall p^{\prime} \in X^{*}, \quad g^{b}\left(p^{\prime}, s^{\prime}\right)+t H\left(p^{\prime}, s^{\prime}\right) \geq p^{\prime} . x
$$

and $v_{b}(x, t) \leq r$. When $H(\cdot, s)$ is sublinear and finite at some point of $\operatorname{dom} g^{*}$, we pick $a \in \operatorname{dom} H(\cdot, s) \cap \operatorname{dom} g^{*}$ (with $b \leq-g^{*}(a)$, so that $g(x) \geq a . x+b$ for every $x \in X$ ) and we use the inequality $\lambda^{-1} H(a, s)+H\left(p^{\prime}, s\right) \geq$ $H\left(\lambda^{-1} a+p^{\prime}, s\right)$ to get the same conclusion. When $H$ is right lower regular, we pick sequences $\left(\lambda_{n}\right) \rightarrow \infty$, $\left(\left(p_{n}, s_{n}\right)\right) \rightarrow\left(p, r_{+}\right)$such that $H(p, r) \geq \lim H\left(p_{n}, s_{n}\right)$, we set $s=r_{n}, p_{n}^{\prime}:=p_{n}-\lambda_{n}^{-1} a$ and we use the fact that there exists some $k>0$ such that $\left|g^{b}\left(p_{n}^{\prime}, s^{\prime}\right)-g^{b}\left(p, s^{\prime}\right)\right| \leq k\left\|p_{n}^{\prime}-p\right\|$ for $s^{\prime} \in\left[r, r^{\prime}\right]$ to pass to the limit in the inequality

$$
g^{b}\left(p_{n}^{\prime}, s_{n}\right)-\lambda_{n}^{-1} b+t H\left(p_{n}, s_{n}\right) \geq\left(\lambda_{n}^{-1} a+p_{n}^{\prime}\right) \cdot x-\lambda_{n}^{-1} r
$$


and get

Thus, in all cases, we have $v_{b}(x, t) \leq r$.

$$
g^{b}\left(p, r_{+}\right)+t H(p, r) \geq p \cdot x
$$

The preceding comparison can be extended to a comparison with the Hopf formula of the convex case.

Proposition 9.3. For every $g: X \rightarrow \overline{\mathbb{R}}, H: X^{*} \rightarrow \overline{\mathbb{R}}$ and every $t>0$ one has $v(\cdot, t):=\left(g^{*}+t H\right)^{*} \leq v_{\%}(\cdot, t):=$ $\left(g^{\%}+t H\right)^{\%}$.

Proof. We first observe that for every $x \in X,(p, q) \in X^{*} \times \mathbb{R}$ we have $g^{\%}(p, q) \leq g^{*}(p)$, so that, when $q>r:=v_{\%}(x, t)$, we have $\left(g^{*}+t H\right)^{*}(x) \leq\left(g_{q}^{\%}+t H\right)^{*}(x) \leq r$.

Example 13. Let $X=\mathbb{R}, g: x \mapsto x_{-}:=\min (x, 0), H=0$. Then, for any $t>0$ one has $v(\cdot, t)=-\infty$ and $v_{\%}(\cdot, t)=g^{\% \%}=g$. The next example shows that the inequality of the preceding proposition may be strict, even when $g$ and $H$ are convex.

Example 14. Let $X=\mathbb{R}, g=0, H=c \in \mathbb{R}$. Then $v(x, t)=-c t$; since $g^{\%}(p, q)=\iota_{\{0\}}(p)$ for $q \geq 0$, $g^{\%}(p, q)=-\infty$ for $q<0$, one has $v_{\%}(\cdot, t)=-c t$ if $c \leq 0, v_{\%}(\cdot, t)=+\infty$ if $c>0$. The following coincidence result dealing with Lax solutions generalizes Example 12.

Proposition 9.4. Let $H: X^{*} \rightarrow \overline{\mathbb{R}}$ be l.s.c. and sublinear. Then, taking $\ell:=H^{b}=H^{\sharp}$, the Lax formula $u_{b}(\cdot, t):=g \diamond \ell_{t}$ coincides with the Lax formula $u(\cdot, t):=g \square(t H)^{*}$ of the convex case.

Proof. Since for any $(x, r) \in X \times \mathbb{R}$ one has $H^{\mathrm{b}}(x) \leq r$ if, and only if, $H^{*}(x) \leq 0$, the function $\ell:=H^{\mathrm{b}}$ is the valley function $v_{S}$ associated with $S:=\left[H^{*} \leq 0\right]$, i.e. $v_{S}(x)=-\infty$ for $x \in S, v_{S}(x)=+\infty$ else, so that $u_{b}(x, t)=\inf _{z \in S} g(x-t z)$. When $H$ is a proper $w^{*}$-l.s.c. sublinear function one has $H^{*}=\iota_{S}$ hence $u(x, t)=\inf _{z \in X}\left(g(x-t z)+\iota_{S}(z)\right)=\inf _{z \in S} g(x-t z)$.

Most comparison results use the local compactness of the space $X$ which is supposed to be finite dimensional ( [13], [18], [75], [119], [120]...). In the following result we use a mean value theorem for some subdifferential $\partial$ ? (see [91] and its references). It is valid provided $X$ satisfies some regularity condition close to the trustworthiness condition of Ioffe [64], [65] called $\partial^{\text {? }}$-reliability in [91]. This condition requires that for any l.s.c. function $f$ on $X$, for any Lipschitzian convex function $g$ on $X$, for any $\varepsilon>0$ and for any $x \in \operatorname{dom} f$ at which $f+g$ attains a local infimum there exist $u, v \in B(x, \varepsilon)$ such that $|f(u)-f(x)|<\varepsilon$ and $0 \in \partial^{?} f(u)+\partial g(v)+\varepsilon B^{*}$, where $B^{*}$ is the unit closed ball of $X^{*}$. This condition is satisfied when $X$ is an Asplund space and $\partial^{\text {? }}$ is the Fréchet subdifferential (or any larger subdifferential such as the Hadamard subdifferential $\partial$ ) or when $X$ has a smooth enough bump function and $\partial^{\text {? }}$ is the viscosity subdifferential.

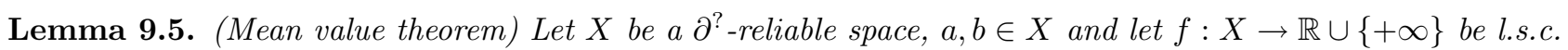
finite at $a \in X$. Then, for every $m \in \mathbb{R}, m<f(b)$ there exist $c \in\left[a, b\left[\right.\right.$ and sequences $\left(c_{n}\right)$, $\left(c_{n}^{*}\right)$ such that $\left(c_{n}\right) \rightarrow c,\left(f\left(c_{n}\right)\right) \rightarrow f(c), c_{n}^{*} \in \partial^{?} f\left(c_{n}\right)$ for each $n \in \mathbb{N}$ and

$$
m-f(a) \leq \liminf _{n}\left\langle c_{n}^{*}, b-a\right\rangle .
$$

Theorem 9.6. Suppose $X \times \mathbb{R}$ is reliable for $\partial^{\text {? }}$. Let $w: X \times \mathbb{P} \rightarrow \mathbb{R}$ be a l.s.c. lower solution to (1) for $\partial^{\text {? }}$ such that for each $x \in X$ one has $\liminf _{(y, t) \rightarrow\left(x, 0_{+}\right)} w(y, t) \leq g(x)$. Then $w \leq u$, the Lax-Oleinik solution with $h=H^{*}$.

Proof. Let $(x, t) \in X \times \mathbb{P}, m \in \mathbb{R}, m<w(x, t)$. For $\left(x^{\prime}, s\right) \in X \times \mathbb{P}$, close enough to $(x, 0)$ we have $m<w\left(x^{\prime}, t+s\right)$. Let us show that for any such $\left(x^{\prime}, s\right)$ and any $y \in X$ we have

$$
m \leq w\left(x^{\prime}-t y, s\right)+t H^{*}(y) .
$$

Since we may suppose $w\left(x^{\prime}-t y, s\right)<+\infty$, this relation follows from the mean value inequality

$$
m-w\left(x^{\prime}-t y, s\right) \leq \liminf _{n}\left(p_{n} . t y+q_{n} t\right)
$$


for some $\left(p_{n}, q_{n}\right) \in \partial^{?} w\left(z_{n}, r_{n}\right)$ where $\left(z_{n}, r_{n}\right)$ is a sequence converging to some $(z, r) \in\left[\left(x^{\prime}-t y, s\right),\left(x^{\prime}, t+s\right)[\right.$ and from the inequalities $q_{n} \leq-H\left(p_{n}\right), p_{n} . y-H\left(p_{n}\right) \leq H^{*}(y)$. Taking the (weak-) limit inferior when $\left(x^{\prime}, s\right) \rightarrow\left(x, 0_{+}\right)$in $(44)$ and using the assumption about the initial condition, we get

$$
m \leq g(x-t y)+t H^{*}\left(\frac{t y}{t}\right)
$$

Taking the infimum on $y$ we get $m \leq u(x, t)$, hence $w(x, t) \leq u(x, t)$.

Using Theorem 6.5, Proposition 8.3 (a) and the fact that $h_{\infty}(0)=0$ when $h$ is l.s.c. proper convex, in particular when $h=H^{*}$, with $H$ a l.s.c. proper convex function, we get the following consequence.

Corollary 9.7. Suppose $X \times \mathbb{R}$ is reliable for the Hadamard subdifferential, $H$ is l.s.c. proper convex and $\operatorname{dom} g^{*} \subset \operatorname{dom} H$. Then the Lax-Oleinik solution is the greatest Hadamard lower solution $w$ of (1) such that $\liminf _{(z, t) \rightarrow(x, 0)} w(z, t) \leq g(x)$.

The following corollary has been obtained in [63] under the additional assumption that dom $H^{*}$ is open. In the remaining part of this section, we suppose $F=H$ and $G=g^{*}$ when considering the convex Hopf-Lax solution.

Corollary 9.8. Suppose $X \times \mathbb{R}$ is reliable for the Hadamard subdifferential, $g$ and $H$ are l.s.c. proper convex functions and $\operatorname{dom} g^{*} \subset \operatorname{dom} H$. Then the Hopf solution is the greatest lower solution $w$ of (1) which is l.s.c.

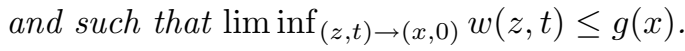

Proof. Since $g$ and $H$ are l.s.c. proper convex functions, the Hopf solution $v$ is the l.s.c. hull of the Lax solution $u$. Since $w \leq u$, and since $w$ is l.s.c., we also have $w \leq v$. Under the additional assumption that $\operatorname{dom} g^{*} \subset \operatorname{dom} H$ we know from Proposition 8.1 (e) that $\liminf _{(z, t) \rightarrow(x, 0)} v(z, t) \leq g(x)$.

The next results will use multi-directional mean value theorems. The simplest one is similar to [35, Thm 2.3 p. 114]; its proof is obtained by adding the use of the lop-sided Moreau minimax theorem to the one in this reference. Here we say that a function $f$ on a normed space $Z$ is tangentially convex if for any $z \in \operatorname{dom} f$ the Hadamard lower derivative $f^{\prime}(z, \cdot)$ is convex. This class contains usual marginal functions.

Lemma 9.9. Let $Z$ be a reflexive Banach space and let $f: Z \rightarrow \mathbb{R} \cup\{+\infty\}$ be a weakly l.s.c. function which is tangentially convex. Given $z_{0} \in Z$ and a bounded closed convex subset $Y$ of $Z$ there exist $z \in \operatorname{co}\left(z_{0}, Y\right)$ and $z^{*} \in \partial f(z)$ such that

$$
\min _{y \in Y} f(y)-f\left(z_{0}\right) \leq \min _{y \in Y} z^{*} \cdot\left(y-z_{0}\right)
$$

Theorem 9.10. Suppose $X$ is reflexive, $F=H: X \rightarrow \mathbb{R}$ is u.s.c. on $\operatorname{dom} g^{*}, G=g^{*}$ and such that $H(\cdot) \leq$ $b+c\|\cdot\|$ for some $b, c \in \mathbb{R}$. Let $w: X \times \mathbb{R}_{+} \rightarrow \mathbb{R}$ be a weakly l.s.c., tangentially convex, Hadamard supersolution to (1) such that $w(\cdot, 0) \geq g$. Then $w \geq v$, the Hopf solution.

Proof. By Proposition 8.1 (b) with $G=g^{*}$ we have $v(\cdot, 0) \leq g \leq w(\cdot, 0)$. Thus it suffices to prove that for any $(x, t) \in X \times \mathbb{P}$, and every fixed $p_{0} \in \operatorname{dom} g^{*}$ one has

$$
f(x, t):=w(x, t)-p_{0} \cdot x+g^{*}\left(p_{0}\right)+t H\left(p_{0}\right) \geq 0 .
$$

Suppose on the contrary that for some $p_{0} \in \operatorname{dom} g^{*}$ there is some $\left(x_{0}, t_{0}\right) \in X \times \mathbb{P}$ such that $f\left(x_{0}, t_{0}\right)<$ 0 . Let $\alpha \in] 0,-f\left(x_{0}, t_{0}\right)\left[\right.$. Since $\inf _{x \in X} f(x, 0) \geq 0$, by Lemma 9.9 , for each $r>0$ there exist $\left(x_{r}, t_{r}\right) \in$ $\operatorname{co}\left(\left(x_{0}, t_{0}\right), B\left(x_{0}, r\right) \times\{0\}\right)$ and $\left(p_{r}, q_{r}\right) \in \partial f\left(x_{r}, t_{r}\right)$ such that, for every $x \in B\left(x_{0}, r\right)$,

$$
\alpha \leq p_{r} \cdot\left(x-x_{0}\right)-q_{r} t_{0} .
$$

The inclusion $\left(p_{r}, q_{r}\right) \in \partial f\left(x_{r}, t_{r}\right)$ is equivalent to the relation $\left(p_{r}+p, q_{r}-H(p)\right) \in \partial w\left(x_{r}, t_{r}\right)$, so that $q_{r}-$ $H(p)+H\left(p_{r}+p\right) \geq 0$. Taking $x \in B\left(x_{0}, r\right)$ such that $p_{r} .\left(x-x_{0}\right)=-r\left\|p_{r}\right\|$, it follows that

$$
\alpha+r\left\|p_{r}\right\| \leq-q_{r} t_{0} \leq t_{0}\left(H\left(p_{r}+p\right)-H(p)\right) .
$$


Let $\rho>0$ be such that $H\left(p+p^{\prime}\right)-H(p)<\alpha / t_{0}$ for $p^{\prime} \in B(0, \rho)$. The preceding inequality ensures that $\left\|p_{r}\right\| \geq \rho$. Using our assumption $H(\cdot) \leq b+c\|\cdot\|$ on the growth of $H$, we get

$$
\alpha+r\left\|p_{r}\right\| \leq t_{0}\left(b+c\left\|p_{r}\right\|+c\|p\|-H(p)\right) .
$$

Thus, for $r>c t_{0}$ we get

$$
\left(r-c t_{0}\right) \rho \leq t_{0}(b+c\|p\|-H(p))-\alpha,
$$

an impossibility when $r$ is large enough.

Note that the growth condition on $H$ is satisfied when $H$ is Lipschitzian.

A more involved mean value inequality will give a variant of the preceding result in which the tangential convexity assumption is dropped.

Lemma 9.11. ([9, Thm 6.1], [66], [28, Thm 3.6.1]) Let $Z$ be a d-reliable Banach space and let $f: Z \rightarrow$ $\mathbb{R} \cup\{+\infty\}$ be a l.s.c. function. Suppose $Y$ is a closed convex subset $Y$ of $Z$ such that $f$ is bounded below on some enlargement $Y+\rho B(0, \varepsilon)$ of $Y$, with $\rho>0$. Given $\varepsilon>0, z_{0} \in Z$ there exist $z \in \operatorname{co}\left(z_{0}, Y\right)+B(0, \varepsilon)$ and $z^{*} \in \partial f(z)$ such that

$$
\sup _{\delta>0} \inf _{y \in Y+B(0, \delta)} f(y)-f\left(z_{0}\right) \leq z^{*} \cdot\left(y-z_{0}\right)+\varepsilon\left\|y-z_{0}\right\| \quad \forall y \in Y .
$$

The comparison result which follows improves Theorem 3.3 in [63] which assumes that $H$ is convex and globally Lipschitzian and that $X$ is a Hilbert space.

Theorem 9.12. Suppose $X$ is reflexive and reliable (for the Hadamard subdifferential), $H$ is u.s.c. on $\operatorname{dom} g^{*}$ and such that $H(\cdot) \leq b+c\|\cdot\|$ for some $b, c \in \mathbb{R}$. Let $w: X \times \mathbb{R}_{+} \rightarrow \mathbb{R}$ be a weakly l.s.c., Hadamard supersolution to (1) such that $w(\cdot, 0) \geq g$. Then $w \geq v$, the Hopf solution.

Proof. Again we prove (45) by supposing on the contrary that for some $p \in \operatorname{dom} g^{*}$ there is some $\left(x_{0}, t_{0}\right) \in$ $X \times \mathbb{P}$ such that $f\left(x_{0}, t_{0}\right)<0$, where, as above, $f(x, t):=w(x, t)-p \cdot x+g^{*}(p)+t H(p)$. Taking $\alpha>0$ with $3 \alpha \leq-f\left(x_{0}, t_{0}\right)$ and using the weak compactness of balls and the weak lower semicontinuity of $f$, for each $r>0$ we can find $s_{r}>0$ such that $f(x, s) \geq-\alpha$ for every $(x, s) \in B\left(x_{0}, r\right) \times\left[0, s_{r}\right]$. Then, taking $\left.\varepsilon \in\right] 0, s_{r}[$ such that $\varepsilon\left\|\left(x, s_{r}\right)-\left(x_{0}, t_{0}\right)\right\|<\alpha$ for every $x \in B\left(x_{0}, r\right)$, Lemma 9.11 yields some $\left(x_{r}, t_{r}\right) \in \operatorname{co}\left(\left(x_{0}, t_{0}\right), B\left(x_{0}, r\right) \times\right.$ $\left.\left\{s_{r}\right\}\right)+B(0, \varepsilon)$ and $\left(p_{r}, q_{r}\right) \in \partial f\left(x_{r}, t_{r}\right)$ such that

$$
\forall x \in B\left(x_{0}, r\right) \quad 2 \alpha \leq p_{r} .\left(x-x_{0}\right)-q_{r} t_{0}+\varepsilon\left\|\left(x, s_{r}\right)-\left(x_{0}, t_{0}\right)\right\| .
$$

Our choice of $\varepsilon$ ensures that $t_{r}>0$ and $\alpha \leq p_{r} .\left(x-x_{0}\right)-q_{r} t_{0}$. Thus, we can finish the proof as in the preceding proof.

Corollary 9.13. Suppose $X$ is reflexive and reliable (for the Hadamard subdifferential) and $H$ is a l.s.c. proper convex function. Suppose $H$ satisfies a linear growth condition: $H(\cdot) \leq b+c\|\cdot\|$ for some $b, c \in \mathbb{R}$. Let $w$ be a l.s.c. function on $X \times \mathbb{R}_{+}$which is convex in its first variable, satisfies $w(x, 0)=\lim _{(z, t) \rightarrow(x, 0)} w(z, t)=g(x)$ for each $x \in X$ and is a supersolution and a lower solution to (1). Then $w=v$, the Hopf solution.

Proof. Under our assumptions, we have $u \geq w \geq v$. Since for each $t>0$ the function $w(\cdot, t)$ is convex and l.s.c. we get $(u(\cdot, t))^{* *} \geq w(\cdot, t)$; since $H=H^{* *}$ we have $(u(\cdot, t))^{* *}=v(\cdot, t)$. It follows that $(u(\cdot, t))^{* *}=w(\cdot, t)=$ $v(\cdot, t)$.

\section{Stability RESUlts}

It is natural to see whether a solution $u$ to (1)-(2) is a limit of solutions to the same system in which $g_{n}, H_{n}$ is substituted to $g, H$ for given sequences $\left(g_{n}\right) \rightarrow g,\left(H_{n}\right) \rightarrow H$; one may also wish to know whether a sequence $\left(u_{n}\right)$ of solutions with given data $g_{n}, H_{n}$ converges to some solution of the system (1)-(2) for the limit data 
$g, H$ of $\left(g_{n}\right),\left(H_{n}\right)$. Such problems are dealt with in a number of places ( [1], [16], [17], [30], [39], [67], [111] ..). Because convergence can be considered in a number of different ways such problems have many different guises.

In this section we supplement the results in [63] by considering perturbation questions. We limit our treatment to the role of duality and we just give a sample of what can be done; see [111] for more. We retain from [63] the idea of considering the Fenchel conjugate of a function with respect to both variables $(x, t)$ :

$$
f^{\star}(p, q):=\sup _{(x, t) \in X \times \mathbb{R}}\{p \cdot x+q t-f(x, t)\} .
$$

Observe that we use a slightly different asterisk for this conjugacy. Let us note the following result which is a variant of [63] Proposition 1.1 in which we do not assume that $g$ and $H$ are l.s.c. proper convex.

Lemma 10.1. Let $v$ and $v_{*}$ be given by $v(x, t):=\left(g^{*}+t H\right)^{*}(x), v_{*}(p, q):=g^{*}(p)+\iota_{\text {epi } H}(p,-q)$. Then the conjugate $v_{*}^{\star}$ of $v_{*}$ for the above conjugacy is given by $v_{*}^{\star}=v$.

Proof. By definition, one has, for any $(x, t) \in X \times \mathbb{R}_{+}$,

$$
\begin{aligned}
v_{*}^{\star}(x, t) & =\sup \left\{p \cdot x+q t-g^{*}(p): p \in \operatorname{dom} H,-q \geq H(p)\right\} \\
& =\sup \left\{p \cdot x-t H(p)-g^{*}(p): p \in \operatorname{dom} H\right\}=v(x, t) .
\end{aligned}
$$

For $(x, t) \in X \times\left(\mathbb{R} \backslash \mathbb{R}_{+}\right)$we have $v_{*}^{\star}(x, t)=+\infty$.

When $H$ is proper l.s.c. convex and $\operatorname{dom} g^{*} \cap \operatorname{dom} H \neq \emptyset$ the function $v_{*}$ is proper l.s.c. convex and we recover [63] Proposition 1.1: $v_{*}(p, q)=v_{*}^{\star \star}(p, q)=v^{\star}(p, q)$. The preceding lemma can be used in addition to continuity results for the Fenchel transform to get some answers to the problems raised above in the case one uses explicit representations ( $[110],[111])$. As a sample of what can be obtained without using explicit representations, let us present the following result.

Proposition 10.2. Let $\left(H_{n}\right)$ be a sequence of proper l.s.c. convex functions on $X^{*}$ and let $H$ be such that $H \leq e-\liminf H_{n}$. Let $\left(w_{n}\right)$ be a sequence of proper l.s.c. convex functions, $w_{n}$ being a lower solution of the equation

$$
\frac{\partial w_{n}}{\partial t}(x, t)+H_{n}\left(D w_{n}(x, t), w_{n}(x, t)\right)=0
$$

Suppose that $\left(w_{n}\right)$ converges in the sense of Mosco to a function $w$. Then $w$ is a lower solution of (1).

In fact, it suffices to have $w \geq e-\lim \sup _{n} w_{n}$ and $w^{\star} \geq e-\lim \sup _{n} w_{n}^{\star}$ for the weak sequential convergence (while, by [6] Theorem 3.19, Mosco convergence amounts to $w \geq e-\lim \sup _{n} w_{n}$ and $w^{\star} \geq e-\lim _{\sup _{n}} w_{n}^{\star}$ for the strong convergence).

Proof. Let $(x, t) \in X \times \mathbb{P}$ and let $(p, q) \in \partial w(x, t)$. By [6] Theorem 3.67 there exists a sequence $\left(\left(x_{n}, t_{n}\right)\right)$ with weak limit $(x, t)$ and a sequence $\left(\left(p_{n}, q_{n}\right)\right) \rightarrow(p, q)$ such that $\left(p_{n}, q_{n}\right) \in \partial w_{n}\left(x_{n}, t_{n}\right)$. By assumption, we have $H_{n}\left(p_{n}\right) \leq-q_{n}$. Since $H \leq e-\liminf H_{n}$ we get $H(p) \leq-q$.

\section{Coincidence of the Hopf and of the Lax solutions}

The coincidence of the formulas giving explicit solutions to the system (1)-(2) is obviouly desirable. Here we restrict ourselves to the convex case; we refer to [4, Thms 6.9-6.11] for the quasiconvex case.

Let us first draw some consequences of the coincidence of $v$ and $u$ at some $(x, t) \in X \times \mathbb{P}$.

Proposition 11.1. Suppose that for some $(x, t) \in X \times \mathbb{P}$ one has $v(x, t)=u(x, t)$. Then, for each $(p, q) \in$ $\partial^{+} u(x, t):=-\partial^{-}(-u)(x, t)$, one has $(p, q) \in \partial^{+} v(x, t)$ and $q+H(p) \geq 0$. Moreover for each $(p, q) \in \partial v(x, t)$ (in particular for each $(p, q) \in \partial^{+} v(x, t)$ ) one has $(p, q) \in \partial u(x, t)$ and if $H(p)=H^{* *}(p)$, then $q+H(p)=0$. 
Proof. Since $u \geq v$, the inclusion $\partial^{+} u(x, t) \subset \partial^{+} v(x, t)$ holds whenever $v(x, t)=u(x, t)$. Since $v$ is convex, for any $(p, q) \in \partial^{+} u(x, t) \subset \partial^{+} v(x, t)$, we have $(p, q) \in \partial v(x, t)$, hence $q+H(p) \geq 0$ by Proposition 4.2.

Let $(p, q) \in \partial v(x, t)=\partial^{c} v(x, t) \subset \partial^{c} u(x, t) \subset \partial u(x, t)$. If $H(p)=H^{* *}(p)$ Theorem 6.6 yields $q+H(p) \leq 0$ while Proposition 4.2 ensures that $q+H(p) \geq 0$.

Now, let us present criteria ensuring that $u$ and $v$ coincide.

Proposition 11.2. Suppose $g$ is convex and $H$ is a l.s.c. proper convex function. Then for each $(x, t) \in X \times \mathbb{P}$ such that $\partial u(\cdot, t)(x) \neq \emptyset$ (or a fortiori $\partial u(x, t) \neq \emptyset$ ) one has $v(x, t)=u(x, t), \partial v(\cdot, t)(x)=\partial u(\cdot, t)(x)$ and $\partial v(x, t)=\partial u(x, t)$.

Proof. Under the assumptions on $g$ and $H$ the function $u$ is convex and $v(\cdot, t)=u(\cdot, t)^{* *}$. For each $(x, t) \in X \times \mathbb{P}$ and each $p \in \partial u(\cdot, t)(x)=\partial^{c} u(\cdot, t)(x)$ the function $u(\cdot, t)$ is lower semicontinuous at $x$, so that one has $u(\cdot, t)(x)=u(\cdot, t)^{* *}(x)=v(\cdot, t)(x)$ and $\partial u(\cdot, t)(x)=\partial^{c} u(\cdot, t)(x)=\partial^{c} u(\cdot, t)^{* *}(x)=\partial^{c} v(\cdot, t)(x)=\partial v(\cdot, t)(x)$. The last assertion is proved similarly.

Proposition 11.3. Suppose $X$ is reflexive, $g$ and $H$ are l.s.c. proper convex functions, and the cone $Z:=$ $\mathbb{R}_{+}\left(\operatorname{dom} g^{*}-\operatorname{dom} H\right)$ is closed and symmetric. Then for each $(x, t) \in X \times \mathbb{P}$ one has $v(x, t)=u(x, t)$. Moreover the infimal convolution in the definition of $u$ is exact.

Proof. This follows from a general result of Attouch and Brézis ( [7]) since in that case one has $\left(g^{*}+t H\right)^{*}=$ $g^{* *} \square(t H)^{*}=g \square h_{t}$. For other criteria in this line see [11].

\section{Conclusion}

We have made no attempt to be complete on the subject of the representation of solutions to first order evolution Hamilton-Jacobi equations. In particular, our treatment of comparison and uniqueness properties is very partial and does not incorporate the results of [39], [40], [120] and others. We have not mentioned any regularizing effect. Such effects have been extensively studied; see [5], [15], [75], [125], [126].

On the other hand, we have strived to shed light on the power of duality for such questions. We also endeavoured to show the analogies between different cases in which convexity or generalized convexity play some role. We hope that such a parallel study will lead to further developments.

Acknowledgements. The authors are most grateful to C. Zălinescu and an anonymous referee for their numerous helpful criticisms.

\section{REFERENCES}

[1] P. Acquistapace and A. Briani, Gamma-convergence for infinite dimensional optimal control problems, in "Evolution equations, Semigroups and Functional Analysis", Lorenzi, Alfredo (ed.) et al., Birkhäuser. Prog. Nonlinear Differ. Equ. Appl. 50, Birkhäuser, Basel (2002) 1-25.

[2] Adimurthi and V. Gowda, Hopf-Lax type formula for sub- and supersolutions, Adv. Differ. Equ. 5, No.1-3 (2000), 97-119.

[3] Adimurthi and V. Gowda, Hopf-Lax type formula for non monotonic autonomous Hamilton Jacobi equations, NoDEA, Nonlinear Differ. Equ. Appl. 11, No.3 (2004), 335-348.

[4] O. Alvarez, E.N. Barron and H. Ishii, Hopf-Lax formulas for semicontinuous data, Indiana Univ. Math. J. 48, No.3 (1999), 993-1035.

[5] M. Arisawa and A. Tourin, Regularizing effects for a class of first-order Hamilton-Jacobi equations, Nonlinear Anal., Theory Methods Appl. 29, No.12 (1997), 1405-1419.

[6] H. Attouch, Variational convergence for functions and operators, Pitman, Boston, (1984).

[7] H. Attouch and H. Brézis, Duality for the sum of convex functions in general Banach spaces. In: Aspects of Mathematics and its applications, J.A. Barroso ed., North Holland, Amsterdam (1986), 125-133.

[8] J.-P. Aubin and H. Frankowska, Set-valued analysis, Birkhäuser, Boston, 1990.

[9] D. Aussel, J.-N. Corvellec and M. Lassonde, Nonsmooth constrained optimization and multidirectional mean value inequalities, SIAM J. Optim. 9, No.3 (1999), 690-706.

[10] D. Azagra, J. Ferrera and F. López-Mesas, Nonsmooth analysis and Hamilton-Jacobi equations on Riemannian manifolds, J. Funct. Anal. 220, No.2 (2005), 304-361.

[11] D. Azé, Duality for the sum of convex functions in general normed spaces, Arch. Math. 62 (1994), 554-561. 
[12] M. Bardi and L.C. Evans, On Hopf's formulas for solutions of Hamilton-Jacobi equations, Nonlinear Anal. 8 (1984), 13731381.

[13] M. Bardi and I. Capuzzo-Dolcetta, Optimal control and viscosity solutions of Hamilton-Jacobi-Bellman equations, Birkhäuser, Basel (1998).

[14] M. Bardi and S. Fiaggian, Hopf-type estimates and formulas for nonconvex nonconcave Hamilton-Jacobi equations, SIAM J. Math. Anal. 29 (1998), 1067-1086.

[15] G. Barles, Regularity results for first-order Hamilton-Jacobi equations, Diff. Integ. Equat. 3 (1990), 103-125.

[16] G. Barles, Solutions de viscosité des équations de Hamilton-Jacobi, Math. et Appl. \#17, Springer, Berlin (1994).

[17] G. Barles and B. Perthame, Discontinuous solutions of deterministic optimal stopping time problems, RAIRO Math. Modeling and Numer. Anal. 21 (1987), 557-579.

[18] E.N. Barron, Viscosity solutions and analysis in $L^{\infty}$, in Nonlinear Analysis, Differential Equations and Control, F.H. Clarke and R.J. Stern (eds.), Kluwer, Dordrecht (1999), 1-60.

[19] E.N. Barron, R. Jensen, Semicontinuous viscosity solutions of Hamilton-Jacobi equations with convex Hamiltonians, Comm. Partial Diff. Eq. 15 (1990), 1713-1742.

[20] E.N. Barron, R. Jensen and W. Liu, Hopf-Lax formula for $v_{t}+H(v, D v)=0$, J. Differ. Eq. 126 (1996), 48-61.

[21] E.N. Barron, R. Jensen and W. Liu, Hopf-Lax formula for $v_{t}+H(v, D v)=0$, II, Comm. P.D.E. 22 (1997), 1141-1160.

[22] E.N. Barron, R. Jensen and W. Liu, Explicit solution of some first order pde's, J. Dynamical and Control Systems 3 (1997), $1-16$.

[23] E.N. Barron and W. Liu, Calculus of variations in $L^{\infty}$, Applied Math. Opt. 35 (1997), 237-243.

[24] E.N. Barron and W. Liu, Semicontinuous solutions for Hamilton-Jacobi equations and the $L^{\infty}$ control problem, Appl. Math. Optim. 34 (1996), 325-360.

[25] E.N. Barron, R. Jensen and W. Liu, Applications of the Hopf-Lax formula for $v_{t}+H(v, D v)=0$, SIAM J. Math. Anal. 29 (1998), 1022-1039.

[26] G. Beer, R.T. Rockafellar and Roger J.-B. Wets, A characterization of epi-convergence in terms of convergence of level sets, Proc. Am. Math. Soc. 116, No.3 (1992), 753-761.

[27] J.M. Borwein and Q.J. Zhu, Viscosity solutions and viscosity subderivatives in smooth Banach spaces with applications to metric regularity, SIAM J. Control Optim. 34 (1996), 1568-1591.

[28] J.M. Borwein and Q.J. Zhu, Techniques of variational analysis, CMS Books in Maths. 20, Canadian Math. Soc., Springer, New York (2005).

[29] A. Braides, $\Gamma$-convergence for beginners, Oxford Lecture Series in Mathematics and its Applications 22, Oxford University Press, Oxford (2002).

[30] A. Briani, Sequences of $L_{\infty}$ optimal control problems: G-convergence and Hamilton-Jacobi equations, preprint, Univ. Pisa (2004).

[31] A. Brondsted and R.T. Rockafellar, On the subdifferentiability of convex functions, Proc. Amer. Math. Soc. 16 (1965), 605-611.

[32] G. Buttazzo, Semicontinuity, relaxation and integral representations in the calculus of variations, Pitman 207, Longman, Harlow (1989).

[33] F. Camilli and A. Siconolfi, Discontinuous solutions of a Hamilton-Jacobi equation with infinite speed of propagation, SIAM J. Math. Anal. 28, No.6 (1997), 1420-1445.

[34] P. Cannarsa and C. Sinestrari, Semiconcave functions, Hamilton-Jacobi equations and optimal control, Birkhäuser, Boston, 2004.

[35] F.H. Clarke, Yu.S. Ledyaev, R.J. Stern and P.R. Wolenski, Nonsmooth analysis and control theory, Springer, 1998.

[36] R. Cominetti, Some remarks on convex duality with and without compactness, Control and Cybernetics, 23, 1-2 (1994), $123-138$.

[37] M.G. Crandall, L.C. Evans and P.-L. Lions, Some properties of viscosity solutions of Hamilton-Jacobi equations, Trans. Amer. Math. Soc. 282 (1984), 487-502.

[38] M.G. Crandall, H. Ishii and P.-L. Lions, User's guide to viscosity solutions of second-order partial differential equations, Bull. Amer. Math. Soc. 27 (1992), 1-67.

[39] M.G. Crandall and P.-L. Lions, Viscosity solutions to Hamilton-Jacobi equations, Trans. Amer. Math. Soc. 277 (1983), 1-42.

[40] M.G. Crandall and P.-L. Lions, Hamilton-Jacobi equations in infinite dimensions, Part I, Uniqueness of viscosity solutions, J. Funct. Anal. 62 (1985), 379-396, Part II, Existence of viscosity solutions 65 (1986) 368-405; Part III 68 (19) 214-247; Part IV Unbounded linear terms 90 (1990), 237-283; Part V B-continuous solutions, 97 (1991), 417-465.

[41] J.-P. Crouzeix, Polaires quasi-convexes et dualité, C.R. Acad. Sci. Paris série A 279 (1974), 955-958.

[42] J.-P. Crouzeix, Contribution à l'étude des fonctions quasi-convexes, Thèse d'Etat, Univ. de Clermont II, 1977.

[43] J.-P. Crouzeix, Some differentiability properties of quasiconvex functions on $\mathbb{R}^{n}$, in "Optimization and optimal control, Proceedings Conference Oberwolfach 1980", A. Auslender, W. Oettli and J. Stoer, eds. Lecture Notes in Control and Information Sciences 30, Springer-Verlag (1981), 89-104.

[44] J.-P. Crouzeix, Continuity and differentiability properties of quasiconvex functions on $\mathbb{R}^{n}$, in "Generalized concavity in optimization and economics", S. Schaible and W.T. Ziemba, eds. Academic Press, New York, (1981), 109-130. 
[45] G. Dal Maso, Introduction to Г-convergence, Birkhäuser, Basel, Boston, 1993.

[46] G. Dal Maso and H. Frankowska, Value functions for Bolza problems with discontinuous Lagrangians and Hamilton-Jacobi inequalities, ESAIM: Control, Optim. Calculus of Variations, 5 (2000), 369-393.

[47] R. Deville, Stability of subdifferentials of nonconvex functions in Banach spaces, Set-Valued Anal. 2, No.1-2 (1994), 141-157.

[48] R. Deville, Smooth variational principles and nonsmooth analysis in Banach spaces, in Nonlinear Analysis, Differential Equations and Control, F.H. Clarke and R.J. Stern (eds.), Kluwer, Dordrecht, (1999), 369-405.

[49] E. El Haddad and R. Deville, The viscosity subdifferential of the sum of two functions in Banach spaces. I First order case, J. Convex Anal. 3 (1996), 295-308.

[50] L.C. Evans, Some max-min methods for Hamilton-Jacobi equations, Indiana Univ. Math. J. 33 (1984), 31-50.

[51] L.C. Evans, Partial differential equations, Graduate Studies in Math. \#19, Amer. Math. Soc., Providence (1998).

[52] F. Ferro, Lower semicontinuity, optimization and regularizing extensions of integral functionals, SIAM J. Control Optim. 19 (1981), 433-444.

[53] F. Ferro, Lower semicontinuity of integral functionals and applications, Boll. Un. Mat. Ital. I-B (1982), 753-763.

[54] H. Frankowska, On the single-valuedness of Hamilton-Jacobi operators, Nonlinear Anal. Th. Methods Appl. 10 (1986), 14771483.

[55] Frankowska, H., Hamilton-Jacobi equations: Viscosity solutions and generalized gradients, J. Math. Anal. Appl. 141, No.1, 21-26 (1989).

[56] H. Frankowska, Optimal trajectories associated with a solution of the contingent Hamilton-Jacobi equation, Applied Math. Optim. 19 (1989), 291-311.

[57] H. Frankowska, Lower semicontinuous solutions of Hamilton-Jacobi-Bellman equations, SIAM J. Control Optim. 31 (1) (1993), 257-272.

[58] G.N. Galbraith, Extended Hamilton-Jacobi characterization of value functions in optimal control, SIAM J. Control Optim. 39 (1) (2000), 281-305.

[59] J.M. Gutiérrez, Infragradientes y direcciones de decrecimiento, Rev. Real Acad. C. Ex., Fis. y Nat. Madrid 78 (1984), 523-532.

[60] E. Hopf, Generalized solutions to non linear equations of first order, J. Math. Mech. 14 (1965), 951-974.

[61] C. Imbert, Convex analysis techniques for Hopf-Lax formulae in Hamilton-Jacobi equations, J. Nonlinear Convex Anal. 2 , No.3 (2001), 333-343.

[62] C. Imbert, A non-local regularization of first order Hamilton-Jacobi equations, J. Differ. Equations 211, No.1 (2005), $218-246$.

[63] C. Imbert and M. Volle, On vectorial Hamilton-Jacobi equations, Control and Cybernetics, 31, No 3 (2002), 493-506.

[64] A.D. Ioffe, Sur la semicontinuité des fonctionnelles intégrales, C.R. Acad. Sci. Paris A 284 (1977), 807-809.

[65] A.D. Ioffe, On lower semicontinuity of integral functionals I,II, SIAM J. Control Optim. 15 (1977), 521-538 and 991-1000.

[66] A.D. Ioffe, Fuzzy principles and characterization of trustworthiness, Set-Valued Anal. 6, No.3 (1998), 265-276.

[67] H. Ishii, Perron's methods for Hamilton-Jacobi equations, Duke Math. J. 55 (1987), 369-384.

[68] H. Ishii, A generalization of a theorem of Barron and Jensen and a comparison theorem for lower semicontinuous viscosity solutions. Proc. Roy. Soc. Edinburgh Sect. A 131 (2001), no. 1, 137-154.

[69] H. Ishii, Representation of solutions of Hamilton-Jacobi equations, Nonlin. Anal. 12 (1988), 121-146.

[70] R. Janin, On sensitivity in an optimal control problem, J. Math. Anal. Appl. 60 (1977), 631-657.

[71] S.N. Kružkov, On the minmax representation of solutions of first order nonlinear equations, Functional Anal. Appl. 2 (1969), $128-136$.

[72] R. Laraki, Repeated games with lack of information on one side: the dual differential approach, preprint, Laboratoire d'économétrie, Ecole Polytechnique, Paris, May 1999.

[73] P.-J. Laurent, Approximation et optimisation, Hermann, Paris, 1972.

[74] P.D. Lax, Hyperbolic systems of conservation laws II, Commun. Pure Appl. Math. 10 (1957), 537-566.

[75] P.-L. Lions, Generalized solutions of Hamilton-Jacobi equations, Research Notes in Math. \#69, Pitman, London (1982).

[76] P.-L. Lions and C. Rochet, Hopf formula and multi-time Hamilton-Jacobi equations, Proc. Amer. Math. Soc. 90 (1980), $79-84$.

[77] P.-L. Lions and P.E. Souganidis, Differential games, optimal control and directional derivatives of viscosity solutions of Bellman's and Isaac's equations, SIAM J. Control and Optim. 23 (1985), 566-583.

[78] Ph. Loewen, Optimal Control via Nonsmooth Analysis, CRM Proceedings and Lecture Notes \# 2, Amer. Math. Soc., Providence, 1993.

[79] J.-E. Martínez-Legaz, On lower subdifferentiable functions, in Trends in Mathematical Optimization, K.H. Hoffmann et al. eds, Int. Series Numer. Math. 84 Birkhauser, Basel, 1988, 197-232.

[80] J.-E. Martínez-Legaz, Quasiconvex duality theory by generalized conjugation methods, Optimization, 19 (1988) 603-652.

[81] J.-E. Martínez-Legaz and I. Singer, Dualities between complete lattices, Optimization 21 (1990), 481-508.

[82] J.-E. Martínez-Legaz and I. Singer, Some characterizations of $\varphi$-Lagrangian dual problems, Optimization 22 (1991), 835-843.

[83] J.-E. Martínez-Legaz and I. Singer, V-dualities and $\perp$-dualities, Optimization 22 (1991), 483-511.

[84] J.-E. Martínez-Legaz and I. Singer, *-dualities, Optimization 30 (1994), 295-315.

[85] Ş. Mirica, Constructive dynamic programming in optimal control. Autonomous problems, Editura Academiei Române, Bucharest 2004. 
[86] J.-J. Moreau, Théorème inf-sup, C.R. Acad. Sci. Paris 258 (1964), 2720-2722.

[87] O.A. Oleinik, The Cauchy problem for nonlinear equations in a class of discontinuous functions, Amer. Math. Soc. Transl. Ser. 2, 42 (1964), 7-12.

[88] N. Papara, On the dynamic programming principle for optimal control problems. Rev. Anal. Numer. Theor. Approx. 27 (1998), no. 2, 315-320.

[89] J.-P. Penot, A remark on the direct method of the calculus of variations, Proc. Amer. Math. Soc. 67 (1977), $135-141$.

[90] J.-P. Penot, On the interchange of subdifferentiation and epi-convergence, J. Math. Anal. Appl. 196 (1995), $676-698$.

[91] J.-P. Penot, A mean value theorem for small subdifferentials, J. Optim Th. Appl. 94 (1) (1997), $209-221$.

[92] J.-P. Penot, Proximal mappings, J. Approx. Th. 94 (1998), 203-221.

[93] J.-P. Penot, Are generalized derivatives useful for generalized convex functions? in "Generalized Convexity, Generalized Monotonicity", J.-P. Crouzeix, J.-E. Martínez-Legaz and M. Volle (eds.) Kluwer, Dordrecht (1998), 3-60.

[94] J.-P. Penot, What is quasiconvex analysis? Optimization 47 (2000), 35-110.

[95] J.-P. Penot, Questions and observations about Hamilton-Jacobi evolution equations, in "Processus optimaux, phénomènes de propagation et équations d'Hamilton-Jacobi", Paris 2-4 octobre 2000, INRIA (2000), 73-89.

[96] J.-P. Penot, The compatibility with order of some subdifferentials, Positivity 6 (4), (2002), 413-432.

[97] J.-P. Penot, The use of nonsmooth analysis and duality methods for the study of Hamilton-Jacobi equations, in "Optimization and Control with Applications." Liqun Qi, Koklay Teo and Xiaoqi Yang (eds.), Springer, New York (2005), 127-140.

[98] J.-P. Penot, The bearing of duality on microeconomics, Adv. in Math. Economics 7 (2005), 113-139.

[99] J.-P. Penot and M. Volle, Dualité de Fenchel et quasi-convexité, C.R. Acad. Sc. Paris série I, 304 (13) (1987), $269-272$.

[100] J.-P. Penot and M. Volle, Another duality scheme for quasiconvex problems, Trends in Mathematical Optimization, K.H. Hoffmann et al. eds, Int. Series Numer. Math. 84 Birkhauser, Basel, 1988, 259-275.

[101] J.-P. Penot and M. Volle, On quasi-convex duality, Math. Oper. Research 15 (4) (1990), 597-625.

[102] J.-P. Penot and M. Volle, Inversion of real-valued functions and applications, Z.O.R. Methods and Models Oper. Research 34 (4) (1990), 117-141.

[103] J.-P. Penot and M. Volle, Topological stability results about approximate solutions of parametrized minimization problems, Optimization 22 (6) (1991), 855-868.

[104] J.-P. Penot and M. Volle, Explicit solutions to Hamilton-Jacobi equations under mild continuity and convexity assumptions, J. Convex and Nonlinear Anal. 1 (2) (2000), 177-199.

[105] J.-P. Penot and M. Volle, Convexity and generalized convexity methods for the study of Hamilton-Jacobi equations, Proceedings of the Sixth Conference on Generalized Convexity and Generalized Monotonicity, Samos, Sept. 1999, N. Hadjisavvas, J.-E. Martínez-Legaz, J.-P. Penot, eds., Lecture Notes in Economics and Math. Systems 502, Springer, Berlin (2001), $294-316$.

[106] J.-P. Penot and C. Zălinescu, Harmonic sum and duality, J. Convex Anal. 7 (1) (2000), 95-113.

[107] J.-P. Penot and C. Zălinescu, Elements of quasiconvex analysis, J. Convex Anal. 7 (2) (2000), $243-269$.

[108] J.-P. Penot and C. Zălinescu, Approximation of functions and sets, in "Approximation, Optimization and Mathematical Economics", M. Lassonde, ed., Physica-Verlag, Heidelberg (2001), 255-274.

[109] J.-P. Penot and C. Zălinescu, Bounded (Hausdorff) convergence: basic facts and applications, in "Variational Analysis and Applications", Proceedings of Erice Conference 2003, F. Giannessi and A. Maugeri, eds., Kluwer, Dordrecht (2005), 827-853.

[110] J.-P. Penot and C. Zălinescu, Continuity of the Legendre-Fenchel transform for some variational convergences, Optimization $53(5-6)(2004), 549-562$.

[111] J.-P. Penot and C. Zălinescu, Persistence and stability of explicit solutions of Hamilton-Jacobi equations, preprint, Univ. of Pau.

[112] P. Plazanet, Contributions à l'analyse des fonctions convexes et des différences de fonctions convexes. Application à l'optimisation et à la théorie des E.D.P., thesis, Univ. P. Sabatier, Toulouse, 1990.

[113] R.T. Rockafellar and R. J-B. Wets, Variational Analysis, Springer-Verlag, Berlin, 1997.

[114] R.T. Rockafellar and P.R. Wolenski, Convexity in Hamilton-Jacobi theory. I. Dynamics and duality, SIAM J. Control Optim. 39 (2000), no. 5, 1323-1350.

[115] R.T. Rockafellar and P.R Wolenski, Convexity in Hamilton-Jacobi theory. II. Envelope representations, SIAM J. Control Optim. 39 (2000), no. 5, 1351-1372.

[116] A. Siconolfi, A first order Hamilton-Jacobi equation with singularity and the evolution of level sets, Commun. Partial Differ. Equations 20, No.1-2 (1995), 277-307.

[117] A. Siconolfi, Almost continuous solutions of geometric Hamilton-Jacobi equations, Ann. Inst. Henri Poincaré, Anal. Non Linéaire 20, No.2 (2003), 237-269.

[118] P.E. Souganidis, Existence of viscosity solutions of Hamilton-Jacobi equations, J. Diff. Equations 56 (1985), 345-390.

[119] T. Strömberg, On viscosity solutions of the Hamilton-Jacobi equation, Hokkaido Math. J. 28, No.3 (1999), $475-506$.

[120] T. Strömberg, The Hopf-Lax formula gives the unique viscosity solution, Differ. Integral Equ. 15, No.1 (2002), 47-52.

[121] T. Strömberg, Hamilton-Jacobi equations having only action functions as solutions, Arch. Math. 83, No.5 (2004), 437-449.

[122] A.I. Subbotin, Generalized solutions of first-order PDE's, Birkhäuser, Basel, 1995.

[123] R. Vinter, Optimal control, Birkhäuser, Boston, 2000.

[124] M. Volle, Convergence en niveaux et en épigraphes, C.R. Acad. Sci. Paris 299 (8) (1984), 295-298. 
[125] M. Volle, Compléments sur la relation entre la régularisation de Lasry-Lions et l'équation de Hamilton-Jacobi, Travaux du Séminaire d'Anal. Convexe, Montpellier (1990), exposé $\mathrm{n}^{\circ} 7$.

[126] M. Volle, Régularisation des fonctions fortement minorées dans les espaces de Hilbert, Travaux du Séminaire d'Anal. Convexe, Montpellier (1990), exposé $\mathrm{n}^{\circ} 8$.

[127] M. Volle, Duality for the level sum of quasiconvex functions and applications, ESAIM: Control, Optimisation and Calculus of Variations, 3 (1998), 329-343, http://www.emath.fr/cocv/

[128] M. Volle, Conditions initiales quasiconvexes dans les équations de Hamilton-Jacobi, C.R. Acad. Sci. Paris série I, 325 (1997), $167-170$.

[129] P.R. Wolenski and Yu Zhuang, Proximal analysis and the minimal time function, SIAM J. Control Optimization 36, No.3 (1998), 1048-1072.

[130] T. Zolezzi, Convergence of generalized gradients, Set-Valued Anal. 2 (1994), 381-393. 